\title{
Variational Convergence for Functionals of Ginzburg-Landau Type
}

\author{
G. Alberti, S. Baldo, \& G. Orlandi
}

This paper is dedicated to the memory of Ennio De Giorgi

\begin{abstract}
In the first part of this paper we prove that certain functionals of Ginzburg-Landau type for maps from a domain in $\mathbb{R}^{n+k}$ into $\mathbb{R}^{k}$ converge in a suitable sense to the area functional for surfaces of dimension $n$ (Theorem 1.1). In the second part we modify this result in order to include Dirichlet boundary condition (Theorem 5.5), and, as a corollary, we show that the rescaled energy densities and the Jacobians of minimizers converge to minimal surfaces of dimension $n$ (Corollaries 1.2 and 5.6). Some of these results were announced in [2].
\end{abstract}

\section{INTRODUCTION}

In this paper we investigate the asymptotic behaviour, as $\varepsilon \rightarrow 0$, of the functionals

$$
F_{\varepsilon}(u):=\int_{\Omega} \frac{1}{k}|D u|^{k}+\frac{1}{\varepsilon^{2}} W(u),
$$

where $\Omega$ is a regular domain in $\mathbb{R}^{n+k}$, with $n \geq 0, k \geq 2$, the map $u: \Omega \rightarrow \mathbb{R}^{k}$ belongs to the Sobolev space $W^{1, k}$, and $W$ is a positive continuous potential on $\mathbb{R}^{k}$ which vanishes only on the unit sphere $S^{k-1}$.

In particular, for $k=2, n=0$, and $W(u)=\frac{1}{4}\left(1-|u|^{2}\right)^{2}$, we recover the complex Ginzburg-Landau functional in the simplified form considered by 
F. Bethuel, H. Brezis, and F. Hélein [9]. This functional has been widely studied in recent years because it shares many of the relevant features of more complex functionals used to model vortices in superfluidity and superconductivity (see [11, 26]).

We focus our attention on some asymptotic properties of the minimizers $u_{\varepsilon}$ of $F_{\varepsilon}$ with prescribed boundary datum $v$ in the trace space $W^{1-1 / k, k}\left(\partial \Omega, S^{k-1}\right)$. Note that this boundary condition has no physical meaning, but is rather a replacement for the additional terms which appear in the physical functionals (e.g., accounting for the interaction with an external magnetic field). If $n \geq 1$ and the Jacobian of the boundary datum $v$ is non-trivial (cf. Section 5.2), the energy of minimizers $F_{\varepsilon}\left(u_{\varepsilon}\right)$ is of order $|\log \varepsilon|$ as $\varepsilon \rightarrow 0$, and the energy densities

$$
e_{\varepsilon}\left(u_{\varepsilon}\right):=\frac{1}{k}\left|D u_{\varepsilon}\right|^{k}+\frac{1}{\varepsilon^{2}} W\left(u_{\varepsilon}\right)
$$

concentrate on a minimal surface of dimension $n$ in $\Omega$.

From a variational viewpoint, this energy-concentration result can be viewed as a corollary of a more general fact: as $\varepsilon \rightarrow 0$, the functionals $F_{\varepsilon}$ approximate in a suitable sense the area functional for surfaces of dimension $n$ (and codimension $k$ ) in $\Omega$. This fact is well-known for the scalar Ginzburg-Landau functionals, namely those defined as in (1.1) for $k=1, u: \Omega \subset \mathbb{R}^{N} \rightarrow \mathbb{R}$, and $W$ a double-well potential on $\mathbb{R}$; a rigorous justification of their convergence to the area functional for codimension-one surfaces was given in terms of $\Gamma$-convergence by $\mathrm{L}$. Modica and S. Mortola [32,33] (see also [1, 5,7,31]), who developed an early suggestion of E. De Giorgi.

In this paper we prove a $\Gamma$-convergence result for the functionals $F_{\varepsilon}$ with $k \geq 2$ and $n \geq 0$, which is very close in spirit to that of Modica and Mortola. ${ }^{1}$

The underlying idea is that the energy densities of the maps $u_{\varepsilon}$ are closely connected to their Jacobians - in other words the Jacobian is the right tool to track energy concentration in (vector) Ginzburg-Landau functionals. We recall that the Jacobian of a map $u \in W^{1, k}\left(\Omega, \mathbb{R}^{k}\right)$ is the $k$-form $J u:=d u_{1} \wedge \cdots \wedge$ $d u_{k}$, where each $d u_{i}$ is the differential of the $i$-th component of $u$ (in particular, for $\left.n=0, J u=\operatorname{det}(D u) d x_{1} \wedge \cdots \wedge d x_{k}\right)$. If we denote by $\star$ the standard identification of $k$-covectors and $n$-vectors in $\mathbb{R}^{n+k}$, then $\star J u$ is an $L^{1}$ map on $\Omega$ valued in $n$-vectors, and defines an $n$-dimensional current in $\Omega$; moreover, $J u$ is a differential, and this means that $\star J u$ is a boundary (see Section 2.10). Thus the Jacobian is naturally endowed with a structure of oriented $n$-dimensional surface in a generalized sense; of course, being an $L^{1}$ function, it brings little resemblance to the usual notion of $n$-dimensional surface, and should be rather understood as a sort of diffuse surface.

However, given maps $u_{\varepsilon}$ such that $F_{\varepsilon}\left(u_{\varepsilon}\right)$ is of order $|\log \varepsilon|$, the corresponding Jacobians $\star J u_{\varepsilon}$ converge, up to a subsequence, to a $n$-dimensional integral

\footnotetext{
${ }^{1}$ Our results do not quite fit in the standard definition of $\Gamma$-convergence as given by $\mathrm{E}$. De Giorgi and T. Franzoni [18] (see also [1, 14, 17]), and we do not rely on any abstract result from that theory.
} 
boundary, that is, a current supported on an $n$-dimensional rectifiable set $M$, which is also a boundary. We prove indeed the following compactness and $\Gamma$ convergence result. ${ }^{2}$

Theorem 1.1. Let $\Omega$ be a bounded Lipschitz domain in $\mathbb{R}^{n+k}$ with $n \geq 0$ and $k \geq 2$. Then the following statements hold.

(i) Compactness and lower-bound inequality. Given a (countable) sequence of maps $\left(u_{\varepsilon}\right)$ such that $F_{\varepsilon}\left(u_{\varepsilon}\right) \leq C|\log \varepsilon|$ for some finite $C$, we can extract a subsequence (not relabelled) such that the Jacobians $\star J u_{\varepsilon}$ converge in the flat norm $\mathbf{F}_{\Omega}$ to $\alpha_{k} M$, where $M$ is an $n$-dimensional integral boundary in $\Omega$, and

$$
\liminf _{\varepsilon \rightarrow 0} \frac{F_{\varepsilon}\left(u_{\varepsilon}\right)}{|\log \varepsilon|} \geq \beta_{k}\|M\|
$$

(ii) Upper bound inequality. For every $n$-dimensional integral boundary $M$ in $\Omega$ there exist maps $u_{\varepsilon}$ such that $\mathbf{F}_{\Omega}\left(\star J u_{\varepsilon}-\alpha_{k} M\right) \rightarrow 0$ and

$$
\lim _{\varepsilon \rightarrow 0} \frac{F_{\varepsilon}\left(u_{\varepsilon}\right)}{|\log \varepsilon|}=\beta_{k}\|M\|
$$

Remarks. (i) It is important to notice that the constant $\beta_{k}$ in the upper and lower bound inequality does not depend on the choice of the potential $W$. Thus, if we replace $W$ by $\sigma W$ in (1.1) and let $\sigma$ tend to 0 in the lower bound inequality (1.3), we obtain the stronger estimate

$$
\liminf _{\varepsilon \rightarrow 0} \frac{1}{|\log \varepsilon|} \int_{\Omega} \frac{1}{k}\left|D u_{\varepsilon}\right|^{k} \geq \beta_{k}\|M\| .
$$

In particular, if the sequence $\left(u_{\varepsilon}\right)$ satisfies the upper bound inequality (1.4), then the potential part of the energy is asymptotically negligible, that is

$$
\int_{\Omega} \frac{1}{\varepsilon^{2}} W\left(u_{\varepsilon}\right)=o(|\log \varepsilon|) .
$$

(ii) As shown in Lemma 3.7 below, the sequence $\star J u_{\varepsilon}$ is asymptotically equivalent in the flat norm $\mathbf{F}_{\Omega}$ to the sequence $\rho\left(u_{\varepsilon}\right) \star J u_{\varepsilon}$ for every bounded function $\rho \in C^{1}\left(\mathbb{R}^{k}\right)$ whose integral on the unit ball of $\mathbb{R}^{k}$ is $\alpha_{k}$, and therefore they converge to the same limit $\alpha_{k} M$.

(iii) For $k=2$ and $n$ arbitrary, i.e., for maps valued in $\mathbb{R}^{2}$, the compactness and lower bound inequality for the functionals $F_{\varepsilon}$ have been proved independently by R.L. Jerrard and H.M. Soner in [29].

\footnotetext{
${ }^{2}$ In what follows, $\alpha_{k}$ is the Lebesgue measure of the unit ball of $\mathbb{R}^{k}, \beta_{k}:=(k-1)^{k / 2} \alpha_{k}$, and $\|M\|$ is the mass of $M$; see Section 2 for further details and the precise definitions of Lipschitz domain, integral boundary and flat norm.
} 
(iv) The convergence of $\star J u_{\varepsilon}$ to $\alpha_{k} M$ in the flat norm implies strong convergence in the dual of forms of class $C_{0}^{1}$ (cf. Section 2.5). By a simple interpolation argument (cf. [29, Theorem 3.5]), one can derive strong convergence of $\star J u_{\varepsilon}$ in the dual of forms of class $C_{0}^{0, \beta}$ for every $\beta>0$. However, the Jacobians $\star J u_{\varepsilon}$ are not necessarily uniformly bounded in mass, ${ }^{3}$ and therefore convergence may not extend to forms of class $C_{0}^{0}$.

(v) Theorem 1.1 should hold also when $\Omega$ is an $(n+k)$-dimensional, smooth, compact manifold with boundary. However, while statement (i) can be easily deduced from the flat case via a localization argument, the proof of statement (ii) requires a careful (even though not difficult) extension of Theorem 5.10 of [3].

(vi) While the exponent $k$ in (1.1) has a relevant geometric meaning-cf. Remark (iv) after Corollary 1.2-the exponent 2 has none: replacing the term $\varepsilon^{-2}$ in front of $W(u)$ by any negative power of $\varepsilon$ would only change the righthand side of (1.3) and (1.4) by a constant factor.

(vii) A different way of stating the convergence of $F_{\varepsilon}$ to the area functional is the following: for a certain $k-1<p<k$, the rescaled functionals $|\log \varepsilon|^{-1} F_{\varepsilon}$ $\Gamma$-converge on $W^{1, p}$, endowed with the weak topology, to the functional which agrees with $\|J u\|$ for $u \in W^{1, p}\left(\Omega, S^{k-1}\right)$ and with $+\infty$ otherwise. For $n=0$ and $k=2$ this statement was actually proved in [29, Theorem 4.1]. For general $n$, the proof of the upper bound inequality would require a much more delicate construction than Theorem 1.1. Note that such a result makes sense only if coupled with the compactness of minimizers in $W^{1, p}$, which is rather difficult to prove (see remark (vi) after Corollary 1.2). On the other hand, the $\Gamma$-limit in weaker topologies is usually trivial: for instance, one easily checks that for every vanishing sequence $\sigma_{\varepsilon}$ and every $1 \leq p<\infty$ the rescaled functionals $\sigma_{\varepsilon} F_{\varepsilon} \Gamma$-converge in the strong topology of $L^{p}$ to the functional which is 0 on $L^{p}\left(\Omega, S^{k-1}\right)$ and $+\infty$ elsewhere.

As stated, Theorem 1.1 has little usefulness, because the minimizers of $F_{\varepsilon}$ on $W^{1, k}\left(\Omega, \mathbb{R}^{k}\right)$ are all constant functions with value in $S^{k-1}$, and there is nothing more to be said about them. However, suitable variants of this result can be used to understand the asymptotics of different problems with non-trivial minimizer. For instance, similar ideas were used in [34] to find non-trivial local minimizers for the Ginzburg-Landau functional in 3d. Another interesting example is the minimization of the Ginzburg-Landau functional for sections of suitable fiber bundles on a given manifold, in the spirit of $[6,7]$.

\footnotetext{
${ }^{3}$ For $n=0, k=2$, and $\Omega=(0,1) \times(0,1)$, set $u_{\varepsilon}\left(x_{1}, x_{2}\right):=\left[1+\varepsilon \cos \left(x_{1} / \delta\right)\right] \cdot \tilde{u}\left(x_{2}\right)$ where $\delta:=\varepsilon|\log \varepsilon|^{-1 / 4}$ and $\tilde{u}$ is a smooth map of $(0,1)$ onto $S^{1}$. Then $F_{\varepsilon}\left(u_{\varepsilon}\right) \sim(\varepsilon / \delta)^{2}=|\log \varepsilon|^{1 / 2}=$ $o(|\log \varepsilon|)$. On the other hand, $u_{\varepsilon}^{-1}(y)$ has cardinality of order $1 / \delta$ for every $y \in \mathbb{R}^{2}$ such that $1-\varepsilon<|y|<1+\varepsilon$, and the area formula yields $\int_{\Omega}\left|J u_{\varepsilon}\right| \sim \varepsilon / \delta=|\log \varepsilon|^{1 / 4}$, which diverges as $\varepsilon \rightarrow 0$.
} 
Following the work of J. Bourgain, H. Brezis, and P. Mironescu [13], in the second half of this paper we consider the minimization of $F_{\varepsilon}$ with a prescribed boundary datum $v$ in the trace space $W^{1-1 / k, k}\left(\partial \Omega, S^{k-1}\right)$. We prove a modification of Theorem 1.1 that includes these Dirichlet boundary conditions (Theorem 5.5) and immediately derive the concentration of Jacobians and energy densities for minimizing sequences (Corollary 5.6) to area-minimizing surfaces of codimension $k$. A particular case of this result is the following.

Corollary 1.2. Let $\Omega$ be a bounded Lipschitz domain in $\mathbb{R}^{n+k}$, with $n \geq 1$ and $k \geq 2$, whose $n$-th homology group $H_{n}(\bar{\Omega}, \mathbb{Z})$ is trivial. Let $v$ be a map in the trace space $W^{1-1 / k, k}\left(\partial \Omega, S^{k-1}\right)$, and for every $\varepsilon>0$ let $u_{\varepsilon}$ be a minimizer of $F_{\varepsilon}$ with trace $v$ on $\partial \Omega{ }^{4}$ Then

$$
\min _{u=v \text { on } \partial \Omega} F_{\varepsilon}(u)=F_{\varepsilon}\left(u_{\varepsilon}\right)=O(|\log \varepsilon|) .
$$

Possibly passing to a subsequence, the Jacobians $\star J u_{\varepsilon}$ converge in the flat norm $\mathbf{F}_{\mathbb{R}^{n+k}}$ to $\alpha_{k} M$, where $M$ minimizes the mass among all $n$-dimensional rectifiable currents supported in $\bar{\Omega}$ with boundary $\partial M=\alpha_{k}^{-1} \star J v$. Moreover, the potential part of the energy is asymptotically negligible in the sense that (1.5) holds, and the energy densities $e_{\varepsilon}\left(u_{\varepsilon}\right)$ and $\left|D u_{\varepsilon}\right|^{k} / k$, rescaled by $1 /|\log \varepsilon|$, converge in the sense of measures to $\beta_{k}|M|$, where $|M|$ is the variation of the vector measure $M$.

Remarks. (i) The boundary of $\star J u_{\varepsilon}$, viewed as a current on the entire $\mathbb{R}^{n+k}$, is $\star J v$ (see Section 5.2). Moreover, $\star J v$ is of the form $\alpha_{k} \partial N$, where $N$ is a rectifiable $n$-dimensional current supported in $\bar{\Omega}$ (see Remark 5.4(ii)) and therefore the minimum problem that defines $M$ is well-posed.

(ii) When $H_{n}(\bar{\Omega}, \mathbb{Z})$ is non-trivial, then $M$ solves the Plateau's problem with boundary condition $\partial M=\alpha_{k}^{-1} \star J v$ in a prescribed cobordism class determined by a generic level set of $u$ (see Corollary 5.6 for a precise statement).

(iii) For $n=0$ it is usually assumed (cf. [9]) that $\partial \Omega$ is connected and the boundary datum $v: \partial \Omega \rightarrow S^{k-1}$ is a smooth map with degree $d \neq 0$. In this case the Jacobians of the minimizers $u_{\varepsilon}$ converge to $\alpha_{k} M$, where $M$ is a 0 -dimensional integral current - a sum of Dirac masses with integer multiplicities $d_{i}$-which minimizes the mass $\|M\|=\sum\left|d_{i}\right|$ under the constraint $\sum d_{i}=d$. Notice that this minimization problem only implies that the integers $d_{i}$ have equal signs, while the locations of the Dirac masses are determined by a lower-order term in the formal expansion of the energies $F_{\varepsilon}$ (see [9] for the case $k=2$ ). Thus the situation is very different from the case $n>0$, where the limit currents $M$ are (almost) completely determined by their minimality property.

\footnotetext{
${ }^{4}$ The existence of such minimizers is ensured by standard semicontinuity and compactness results.
} 
(iv) If the term $|D u|^{k}$ in $F_{\varepsilon}$ is replaced by $|D u|^{p}$, then energy-concentration occurs only for $p \geq k$. Indeed, for every $p>1$ the functionals $F_{\varepsilon}$ are equicoercive and $\Gamma$-converge on $L^{1}$ to the functional $F$ equal to $\int_{\Omega}|D u|^{p}$ for $u \in$ $W^{1, p}\left(\Omega, S^{k-1}\right)$, and $+\infty$ elsewhere. As pointed out in [24], for $p<k$ every map $v$ in $W^{1-1 / p, p}\left(\partial \Omega, S^{k-1}\right)$ is the trace of a map in $W^{1, p}\left(\Omega, S^{k-1}\right)$, and therefore the minimum of $F_{\varepsilon}$ with Dirichlet boundary datum $v$ is of order $O(1)$. Hence the minimizers of $F_{\varepsilon}$ converge weakly in $W^{1, p}$ to minimizers of $F$-that is, $p$-harmonic maps from $\Omega$ into $S^{k-1}$-and convergence carries over to Jacobians and energy densities. On the other hand, for $p \geq k$ not all maps $v$ in $W^{1-1 / p, p}\left(\partial \Omega, S^{k-1}\right)$ are traces of maps in $W^{1, p}\left(\Omega, S^{k-1}\right)$; thus the previous argument does not apply, and in fact we expect a situation very similar to the case $p=k$, albeit with few essential differences: the scaling factor $1 /|\log \varepsilon|$ in Theorem 1.1 should be replaced by $\varepsilon^{p-k}$, and the constant $\beta_{k}$ should be replaced by a different one, determined by a suitable optimal-profile problem. ${ }^{5}$ The fact that for $p=k$ the constant $\beta_{k}$ is not determined by an optimal-profile problem, and the $\Gamma$-limit of the functionals $F_{\varepsilon}$ does not depend on the choice of the potential $W$, is related to the fact that $p=k$ is in some sense a critical case (as shown in [4], a similar situation occurs also for scalar functionals).

(v) Corollary $\mathbf{1 . 2}$ is very close to many concentration results proved in recent years for minimizers of the Ginzburg-Landau functional. For $n=0$ and $k=2$, that is, for maps from $\Omega \subset \mathbb{R}^{2}$ into $\mathbb{R}^{2}$, an exhaustive description of the asymptotic behaviour of minimizers $u_{\varepsilon}$ was given by Bethuel, Brezis, and Hélein [9] (see also $[10,40])$. Some of their results about concentration on points have been later extended in $[22,25]$ to maps from $\Omega \subset \mathbb{R}^{k}$ into $\mathbb{R}^{k}$ with $k$ arbitrary. ${ }^{6}$ For $n=1$, concentration results on curves of minimal length were obtained by T. Rivière [35] in the case $k=2$, and by E. Sandier [38] in the case $k$ arbitrary. Concentration on minimal surfaces of arbitrary dimension $n$ and codimension $k=2$ was proved by F.-H. Lin and T. Rivière [30].

(vi) In the papers mentioned above, it is also shown that the minimizers $u_{\varepsilon}$ are weakly compact in some Sobolev space. For $k=2$ and $n$ arbitrary, an elegant and relatively simple proof of the compactness of critical points of $F_{\varepsilon}$ in $W^{1, p}$ for $p<n /(n-1)$ has been given in [8]. We underline that such compactness results cannot be obtained only by energy methods, but require the full strength of the Euler-Lagrange equation of $F_{\varepsilon}$ (cf. [16]). To our knowledge, no results are available for general $k$ and $n$.

\footnotetext{
${ }^{5}$ The proof of the $\Gamma$-convergence for $p>k$ has been recently carried out in [19].

${ }^{6} \operatorname{In}[9]$ it is shown that, for every $\delta>0$, the sets $\left\{\left|u_{\varepsilon}\right| \leq 1-\delta\right\}$ converge in the sense of Kuratowski to a finite set $S$, where also energy densities concentrate. For $k>2$, energy concentration in a finite set $S$ follows by the weak convergence of $u_{\varepsilon}$ in $W_{\text {loc }}^{1, k}\left(\Omega \backslash S, \mathbb{R}^{k}\right)$ proved in [25] (see also [27]).
} 
To better explain the relation between Jacobians and energy concentration, we briefly sketch the idea of the proof of statement (i) of Theorem 1.1 for maps from $\Omega \subset \mathbb{R}^{k}$ into $\mathbb{R}^{k}$ (i.e., $n=0$ ).

Let maps $u_{\varepsilon}$ be given such that $F_{\varepsilon}\left(u_{\varepsilon}\right)$ is of order $|\log \varepsilon|$, and let $e_{\varepsilon}=e_{\varepsilon}\left(u_{\varepsilon}\right)$ denote the corresponding energy densities. For every $\varepsilon>0$ we choose a cubic grid of size $\ell=\ell(\varepsilon)$, and denote its $(k-1)$-dimensional skeleton by $R_{\varepsilon, k-1}$ (see Figure 3.1 in Section 3). If $\ell$ is chosen so that $\varepsilon|\log \varepsilon|=o(\ell)$ and the grid is suitably positioned, the functions $\left|u_{\varepsilon}\right|$ converge to 1 uniformly on $R_{\varepsilon, k-1}$ as $\varepsilon \rightarrow 0$, and then we can slightly perturb each $u_{\varepsilon}$ so that $\left|u_{\varepsilon}\right|=1$ on $R_{\varepsilon, k-1}$. Now, for every $k$-dimensional cell $Q$ in the grid we denote by $d_{Q}$ the degree of the restriction of $u_{\varepsilon}$ to $\partial Q$ (as a map valued in $S^{k-1}$ ). Then

(i) $\int_{Q} e_{\varepsilon} \geq \beta_{k}\left|d_{Q}\right| \cdot|\log \varepsilon|$ by a fundamental estimate of R.L. Jerrard [27];7

(ii) $\int_{Q} \star J u_{\varepsilon}=\alpha_{k} d_{Q}$. Indeed $\star J u_{\varepsilon}=\operatorname{det}\left(D u_{\varepsilon}\right)$, and by the area formula its integral on $Q$ is equal to the integral of the degree $\operatorname{deg}\left(u_{\varepsilon}, Q, y\right)$ over all $y \in \mathbb{R}^{k}$; on the other hand, $\operatorname{deg}\left(u_{\varepsilon}, Q, y\right)$ is equal to $d_{Q}$ for $|y|<1$ and to 0 for $|y|>1$.

We consider now the measure $\mu_{\varepsilon}:=\sum d_{Q} \delta_{Q}$, where $\delta_{Q}$ is the Dirac mass at the center of $Q$ and the sum is taken over all cells $Q$ in the grid. By (i) we obtain that $|\log \varepsilon|^{-1} F_{\varepsilon}\left(u_{\varepsilon}\right) \geq \beta_{k} \sum\left|d_{Q}\right|=\beta_{k}\left\|\mu_{\varepsilon}\right\|$. Hence the measures $\mu_{\varepsilon}$ converge (up to a subsequence) to a measure $\mu$ which is also a sum of Dirac masses with integer multiplicity, i.e., a 0 -dimensional integral current, and moreover,

$$
\liminf _{\varepsilon \rightarrow 0} \frac{F_{\varepsilon}\left(u_{\varepsilon}\right)}{|\log \varepsilon|} \geq \beta_{k}\|\mu\| .
$$

Statement (ii) above shows that $\star J u_{\varepsilon}$ gets closer and closer to $\alpha_{k} \mu_{\varepsilon}$, in the sense that their integrals over all cells in the grid are the same, and the size of these cells tends to 0 . Indeed $\star J u_{\varepsilon}$ and $\alpha_{k} \mu_{\varepsilon}$ are asymptotically equivalent in the flat norm $\mathbf{F}_{\Omega}$ if $\ell=o\left(|\log \varepsilon|^{-1}\right)$, and therefore $\star J u_{\varepsilon}$ converge to $\alpha_{k} \mu$, too.

Let us briefly comment on the passage to higher dimension $(n>0)$. The key step in the proof sketched above consists in approximating the Jacobians $\star J u_{\varepsilon}$ by the measures $\alpha_{k} \mu_{\varepsilon}$ which are obtained by "projecting" all the mass of $\star J u_{\varepsilon}$ contained in each cell of the grid into its center. Now we realize that this is just Federer-Fleming polyhedral deformation theorem applied to the 0-dimensional current $\star J u_{\varepsilon}$ and to the dual grid, in the sense that $\alpha_{k} \mu_{\varepsilon}$ is the push-forward of $\star J u_{\varepsilon}$ according to a retraction of $\mathbb{R}^{2} \backslash R_{\varepsilon, k-1}$ onto the 0 -dimensional dual skeleton $R_{\varepsilon, 0}^{\prime}$, namely the set of centers of the cells in the original grid (see Figure 3.1 in Section 3).

${ }^{7}$ The estimate actually contains an additional error term that depends on the restriction of $u_{\varepsilon}$ on $\partial Q$. However, the proof can be adjusted so to control this error by the integral of $e_{\varepsilon}$ on $\partial Q$, and this happens to be enough for our purposes. Results along these lines were also obtained in earlier work by E. Sandier [37]. 
Thus, for general $n$ and $k$, we apply a suitable version of the deformation theorem to the $n$-dimensional current $\star J u_{\varepsilon}$, or, more precisely, to a slight perturbation of $\star J u_{\varepsilon}$, and obtain a current $\alpha_{k} M_{\varepsilon}$, where $M_{\varepsilon}$ is a polyhedral boundary with integral multiplicity. Then we show that $\alpha_{k} M_{\varepsilon}$ gets closer and closer to $\star J u_{\varepsilon}$ as $\varepsilon$ tends to 0 , and its mass can be controlled by $|\log \varepsilon|^{-1} F_{\varepsilon}\left(u_{\varepsilon}\right)$. Now the compactness of $M_{\varepsilon}$, and therefore that of $\star J u_{\varepsilon}$, follows from the closure theorem for integral currents, while the lower-bound inequality (1.3) is a by-product of an estimate obtained in the process and a standard localization argument.

The proof of statement (ii) of Theorem 1.1 is a direct application of the construction of $S^{k-1}$-valued maps with prescribed Jacobians described in [3], inspired to the dipole construction of [15]. In the case of prescribed boundary values (Theorem 5.5) compactness and lower bound inequality can be derived (with some work) from statement (i) of Theorem 1.1, but the upper bound inequality must be proved almost from scratch, and requires a non-trivial modification of the construction of [3] (see Appendix B).

The paper is organized as follows:

\section{CONTENTS}

1. Introduction .................................... 1411

2. Preliminaries and Notation .......................... 1418

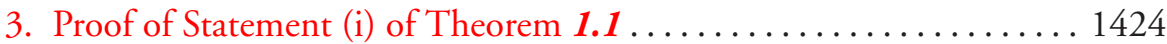

4. Proof of Statement (ii) of Theorem $1.1 \ldots \ldots \ldots \ldots \ldots \ldots \ldots . \ldots \ldots 1437$

5. Boundary Value Problems ......................... 1440

6. Proof of Proposition 5.3 and Theorem 5.5(i) ............. 1444

7. Proof of Theorem 5.5(ii) and Corollary $5.6 \ldots \ldots \ldots \ldots \ldots \ldots . \ldots 1452$

8. Appendix A: Technical Lemmas and Approximation Results ....... 1456

9. Appendix B: Maps With Prescribed Jacobians................ 1462

References ............................................... 1469

\section{PReliminaries AND Notation}

In this paper we use the term sequence also for families $\left(x_{\varepsilon}\right)$ (of points, functions, or else) parametrized by the continuous positive parameter $\varepsilon$, and call countable subsequence any subfamily $\left(x_{\varepsilon_{i}}\right)$, with $i$ integer and $\lim \varepsilon_{i}=0$ as $i \rightarrow+\infty$. In order to simplify the notation, we often omit to relabel subsequences, and simply write $x_{\varepsilon}$ instead of $x_{\varepsilon_{i}}$.

Given functions $f$ and $g$, we write as usual $f=o(g)$ and $f=O(g)$ to mean that $\lim |f| /|g|$ vanishes and $\lim \sup |f| /|g|$ is finite, respectively. We use the letter $K$, with no index, to denote all universal constants, that is, constants that depend only on $k, n$, and on the potential $W$, but not on $\Omega$, $\varepsilon$ or any other parameter; $K$ may take different values even within the same expression. We use the letter $C$, possibly indexed, for constants that are not universal. 
We write $a \vee b$ for $\max \{a, b\}$, and $a \wedge b$ for $\min \{a, b\}$. The symbol $\wedge$ is also used for the wedge product of vectors and covectors, the difference being clear from the context. We write $U \Subset V$ to mean that $U$ is relatively compact in $V$. We denote by $B_{r}=B_{r}^{d}$ the open ball in $\mathbb{R}^{d}$ with center in the origin and radius $r$; for every integer $k \geq 2, S^{k-1}:=\partial B_{1}^{k}$ is the unit sphere in $\mathbb{R}^{k}$, and

$$
\alpha_{k}:=\mathcal{L}^{k}\left(B_{1}^{k}\right), \quad \beta_{k}:=(k-1)^{k / 2} \alpha_{k},
$$

where $\mathcal{L}^{d}$ denotes the Lebesgue measure on $\mathbb{R}^{d} . \mathcal{H}^{h}$ is the $h$-dimensional Hausdorff measure. All other measures $\mu$ are Borel, locally bounded, and possibly vector-valued; $\mu L E$ is the restriction of the measure $\mu$ to the Borel set $E$, while $f \cdot \mu$ is the measure associated to a density function $f \in L_{\text {loc }}^{1}(\mu)$. Sets and functions are always assumed Borel measurable. When no doubts may arise, we omit any explicit mention of the measure in integrals.

Given $h$-dimensional Lipschitz manifolds $N$ and $N^{\prime}$ with $N$ compact and $\partial N^{\prime}=\varnothing$, and a continuous map $f: N \rightarrow N^{\prime}$, we denote by $\operatorname{deg}\left(f, N, N^{\prime}, y\right)$ the degree of $f$ at $y \in N^{\prime} \backslash f(\partial N)$. When $N$ has no boundary and $N^{\prime}$ is connected the degree does not depend on $y$, and we simply write $\operatorname{deg}\left(f, N, N^{\prime}\right)$.

For the rest of this paper, $n \geq 0$ and $k \geq 2$ are given integers, and $\Omega$ is a bounded Lipschitz domain in $\mathbb{R}^{n+k}$. This means that $\partial \Omega$ can be covered by open sets $U$ such that $\Omega$ agrees inside each $U$ with the epigraph of a Lipschitz function (with respect to a suitable choice of the axes). Concerning the regularity of the boundary of $\partial \Omega$, we do not aim to achieve the maximal generality, but rather to show that the concentration phenomena that we investigate-unlike others-are not affected by a mild lack of smoothness in the boundary, including the presence of corner points.

2.1. Ginzburg-Landau functionals. The potential $W$ is a continuous function on $\mathbb{R}^{k}$ which vanishes on $S^{k-1}$, is strictly positive elsewhere, and has growth of order at least two around the zero-set, and at least $k$ at infinity, ${ }^{8}$ that is

$$
\liminf _{|y| \rightarrow 1} \frac{W(y)}{(1-|y|)^{2}}>0 \text { and } \quad \liminf _{|y| \rightarrow \infty} \frac{W(y)}{|y|^{k}}>0
$$

Given $\varepsilon>0, u \in W^{1, k}\left(\Omega, \mathbb{R}^{k}\right)$, and $A \subset \Omega$, we set (cf. (1.1))

$$
F_{\varepsilon}(u, A):=\int_{A} \frac{1}{k}|D u|^{k}+\frac{1}{\varepsilon^{2}} W(u),
$$

\footnotetext{
${ }^{8}$ The growth of order two around the zero set is needed only for a direct application of Jerrard's estimate in the proof of Lemma 3.10, and could be removed with a little extra work. However, a growth of order $k$ around the zero set and at infinity is essential in the proof of Lemma 3.7.
} 
where $|D u|$ stands for the euclidean norm of the matrix $D u{ }^{9}$ The $\varepsilon$-energy density associated to $u$ is

$$
e_{\varepsilon}(u):=\frac{1}{k}|D u|^{k}+\frac{1}{\varepsilon^{2}} W(u) .
$$

2.2. Currents. For currents and forms we follow, with few exceptions, the standard notation (see [39, Chapter 6], or [21, Chapter 2], to which we also refer for a more detailed account of the theory). We just recall here some basic definitions.

For every integer $h=0, \ldots, n+k$, an $h$-form on $\Omega$ is a map from $\Omega$ into the space of $h$-covectors $\Lambda^{h}\left(\mathbb{R}^{n+k}\right)$, while an $h$-dimensional current is a map, or more generally a distribution, valued into the space of $h$-vectors $\Lambda_{h}\left(\mathbb{R}^{n+k}\right)$. Using the standard duality product of vectors and covectors, the space $\mathcal{D}_{h}(\Omega)$ of all $h$-currents is identified with the dual of the space $\mathcal{D}^{h}(\Omega)$ of all smooth $h$ forms with compact support. In particular, the integration of $h$-forms on a given $h$-dimensional oriented surface of class $C^{1}$ defines an $h$-current (often identified with the surface itself).

The boundary of an $h$-current $T$ is the $(h-1)$-current defined by the identity $\partial T[\omega]:=T[d \omega]$ for every $\omega \in \mathcal{D}^{h-1}(\Omega)$, where $d \omega$ is the differential of $\omega$. We call boundary any current which is also a boundary.

A current $T$ is said to have (locally) finite mass when it can be represented as a (locally) bounded Borel measure valued in $\Lambda_{h}\left(\mathbb{R}^{n+k}\right)$; in this case $|T|$ is the variation of the measure $T$ and the mass of $T$ is the total variation $\|T\|:=|T|(\Omega) .{ }^{10}$ We write $\|T\|_{U}:=|T|(U)$ for the mass of $T$ in the open set $U \subset \Omega$. The restriction of $T$ to a set $E$ is denoted by $T\llcorner E$, as for measures.

2.3. Rectifiable and polyhedral currents. A set $M$ in $\Omega$ is $h$-rectifiable if it can be covered, up to an $\mathcal{H}^{h}$-negligible subset, by countably many $h$-surfaces of class $C^{1}$. As such, it admits at $\mathcal{H}^{h}$-a.e. $x \in M$ a tangent space $\operatorname{Tan}(M, x)$ in a measure theoretic sense. An orientation of $M$ is a map which associates to $\mathcal{H}^{h}$-a.e. $x \in M$ a simple unitary $h$-vector which spans $\operatorname{Tan}(M, x) .{ }^{11}$ Given a rectifiable set $M$, an orientation $\tau_{M}$, and a multiplicity $\sigma_{M}$-namely a real function in $L_{\mathrm{loc}}^{1}\left(\mathcal{H}^{h} \mathrm{~L}\right.$

\footnotetext{
${ }^{9}$ The choice of the norm is related to the value of the constant $\beta_{k}$ in Theorem 1.1. It plays an effective role only in estimate (3.16) and computation (4.6).

${ }^{10}$ The spaces $\bigwedge_{h}\left(\mathbb{R}^{n+k}\right)$ and $\bigwedge^{h}\left(\mathbb{R}^{n+k}\right)$ are endowed with the euclidean norms induced by the standard bases. If $v$ is a simple $h$-vector, that is, if $v$ can be written as $v_{1} \wedge \cdots \wedge v_{h}$, then the norm $|v|$ is the $h$-dimensional volume of the parallelogram spanned by the vectors $v_{1}, \ldots, v_{h}$, and agrees with the mass of $v$, as defined in [20, Section 1.8.1]. Note that Jacobians and rectifiable currents are valued in simple vectors, and then the difference between mass and euclidean norm is irrelevant.

${ }^{11}$ If $M$ is a smooth $h$-surface with boundary, the orientation $\tau_{M}$ is always assumed continuous, and the orientation of the boundary satisfies $\eta(x) \wedge \tau_{\partial M}(x)=\tau_{M}(x)$ for every $x \in \partial M$, where $\eta(x)$ is the outer unit normal of $\partial M$ at $x$.
} 
$M)$ —we define the current

$$
T[\omega]:=\int_{M} \sigma_{M}\left(\omega \cdot \tau_{M}\right) d \mathcal{H}^{h} \quad \text { for every } \omega \in \mathcal{D}^{h}(\Omega),
$$

that is, $T:=\sigma_{M} \tau_{M} \cdot \mathcal{H}^{h}\llcorner M$. In this case, the mass of $T$ is just the measure of $M$ counted with multiplicity, that is, $\|T\|=\int_{M}\left|\sigma_{M}\right| d \mathcal{H}^{h}$.

A current $T$ is rectifiable if it can be represented as in (2.3) for some integervalued multiplicity $\sigma_{M}$, and it is integral if both $T$ and $\partial T$ are rectifiable. Notice that a rectifiable boundary $T$ is also integral, because $\partial T=0$. A sum of finitely many $k$-dimensional simplexes in $\mathbb{R}^{n+k}$, endowed with constant orientations and constant real (resp., integral) multiplicities, is a real (resp., integral) polyhedral current in $\mathbb{R}^{n+k}$. Polyhedral currents in $\Omega$ are obtained by restriction.

By the constancy theorem, every $h$-dimensional current without boundary supported on a union $M$ of $h$-dimensional simplexes with pairwise disjoint interiors can be represented as in (2.3) with a multiplicity function which is constant on each simplex, and therefore is a real polyhedral current (cf. [20, Section 4.1.32]).

2.4. Pull-back of forms and push-forward of currents. Given a linear map $L: \mathbb{R}^{n+k} \rightarrow \mathbb{R}^{m}$ and $\beta \in \Lambda^{h}\left(\mathbb{R}^{m}\right)$, then $L^{\sharp} \beta$ is the $h$-covector on $\mathbb{R}^{n+k}$ defined by the identity $\left(L^{\sharp} \beta\right) \cdot\left(v_{1} \wedge \cdots \wedge v_{h}\right)=\beta \cdot\left(L v_{1} \wedge \cdots \wedge L v_{h}\right)$ for every simple vector $v_{1} \wedge \cdots \wedge v_{h}$. Given an open set $\Omega^{\prime}$ in $\mathbb{R}^{m}$ and a map $f: \Omega \rightarrow \Omega^{\prime}$, the pull-back of an $h$-form $\omega$ on $\Omega^{\prime}$ according to $f$ is the $h$-form $f^{\sharp} \omega$ on $\Omega$ defined by $f^{\sharp} \omega(x):=(D f(x))^{\sharp} \omega(f(x))$ for every $x$. The push-forward of an $h$-current $T$ on $\Omega$ is the $h$-current $f_{\sharp} T$ on $\Omega^{\prime}$ defined by the obvious duality $f_{\sharp} T[\omega]=T\left[f^{\sharp} \omega\right]$. If $T$ has compact support in $\Omega$, then $\partial\left(f_{\sharp} T\right)=f_{\sharp}(\partial T)$.

The push-forward has a clear geometric meaning: if $T$ is the current associated $\left(M, \tau_{M}, \sigma_{M}\right)$ as in $(2.3)$, then $f_{\sharp} T$ is the current associated to $\left(M^{\prime}, \tau_{M^{\prime}}, \sigma_{M^{\prime}}\right)$ where $M^{\prime}$ is the rectifiable set $f(M), \tau_{M^{\prime}}$ is any orientation of $M^{\prime}$, and $\sigma_{M^{\prime}}(y)$ is the sum of the multiplicities $\sigma_{M}(x)$ over all $x \in f^{-1}(y)$, computed taking the orientation into account.

2.5. Flat norm. Throughout this paper we mostly deal with currents that are boundaries, or differ by a boundary. Therefore it is convenient to define the following flat norm of a current $T \in \mathcal{D}_{h}(\Omega)$ :

$$
\mathbf{F}_{\Omega}(T):=\inf \left\{\|S\|_{\Omega}: S \in \mathcal{D}_{h+1}(\Omega) \text { and } T=\partial S\right\},
$$

where the infimum is taken to be $+\infty$ if $T$ is not a boundary. We say that $T_{i}$ converge to $T$ in the flat norm $\mathbf{F}_{\Omega}$ if $\mathbf{F}_{\Omega}\left(T_{i}-T\right) \rightarrow 0$. This implies in particular that $T_{i}$ and $T$ differ by a boundary (for $i$ sufficiently large).

Of course, $\mathbf{F}_{\Omega}$ is related to, but not exactly the same as the usual flat seminorms for integral currents (see [20, Section 4.1.24], or [39, Section 31]). Clearly, $\mathbf{F}_{\Omega}(T) \geq T[\omega]$ for every $\omega \in \mathcal{D}^{h}(\Omega)$ such that $\|d \omega\|_{\infty} \leq 1$, and therefore 
convergence in the flat norm $\mathbf{F}_{\Omega}$ implies convergence in the dual of the space of $h$-forms on $\Omega$ of class $C_{0}^{1}$.

The following is a version of the closure theorem for integral currents.

Proposition 2.6 (see Section 8 for the proof). Given a sequence of h-dimension-

al integral boundaries $\left(M_{i}\right)$ in $\Omega$ which are uniformly bounded in mass, and an open set $U \Subset \Omega$, we can extract a subsequence which converges in the flat norm $\mathbf{F}_{U}$ to an integral boundary in $U$.

2.7. Coarea formula for Sobolev maps. Following [3, Section 7.5], we extend the coarea formula to maps $u$ in $W^{1, k}\left(\Omega, \mathbb{R}^{k}\right)$. Let $E$ be the set of points of approximate differentiability of $u$ (more precisely, of the representative of $u$ under consideration) and set $M_{y}=M_{y}(u):=u^{-1}(y) \cap E$ for every $y \in \mathbb{R}^{k}$. Then the sets $M_{y}$ are $n$-rectifiable and have finite $\mathcal{H}^{n}$-measure for a.e. $y$. Moreover, they can be oriented in such a way that the resulting rectifiable $n$-currents on $\mathbb{R}^{n+k}$ —still denoted by $M_{y}$ —satisfy, for every bounded continuous function $\rho: \mathbb{R}^{k} \rightarrow \mathbb{R}$

$$
\begin{gathered}
\|\rho(u) \star J u\|=\int_{\mathbb{R}^{k}}\left\|M_{y}\right\||\rho(y)| d y, \\
\rho(u) \star J u=\int_{\mathbb{R}^{k}} M_{y} \rho(y) d y .
\end{gathered}
$$

The integral in (2.6) is understood in the weak* sense; in other words, identity (2.6) means that

$$
(\rho(u) \star J u)[\omega]=\int M_{y}[\omega] \cdot \rho(y) d y
$$

for every $n$-form $\omega$ on $\mathbb{R}^{n+k}$ of class $C_{0}^{0}$. Note that if $u$ is a Lipschitz map, then $\left\|M_{y}\right\|=\mathcal{H}^{n}\left(u^{-1}(y)\right)$ for a.e. $y$, and setting $\rho=1$ in (2.5) we recover the usual coarea formula.

The map $y \mapsto M_{y}$ is well-defined and approximately continuous in the weak* sense at a.e. $y \in \mathbb{R}^{k}$, meaning that $y \mapsto\left\|M_{y}\right\|$ and $y \mapsto M_{y}[\omega]$ are approximately continuous at $y$ for all $n$-forms $\omega$ on $\mathbb{R}^{n+k}$ of class $C_{0}^{0}$. We call such $y$ regular values, and the corresponding $M_{y}$ regular level sets. In the following we write $M_{y}$ only for regular level sets.

Remark 2.8. (i) If $u$ is a map of class $C^{1}$, every value $y$ which is regular in the classical sense is also regular in our sense, and the corresponding $M_{y}$ is an $n$-surface of class $C^{1}$ oriented by the $n$-vector $\star J u$. By Sard theorem the set of regular values in the classical sense has full measure if $u$ is of class $C^{k+1}$, but it may be even empty for maps of class $C^{k}$.

(ii) For maps in $W^{1, k-1}\left(\Omega, S^{k-1}\right)$, the notion of regular value and regular set is the obvious generalization of the one for maps in $W^{1, k-1}\left(\Omega, \mathbb{R}^{k-1}\right)$. In particular, 
$M_{y}$ is a rectifiable current of dimension $(n+1)$ for $\mathcal{H}^{k-1}$-a.e. $y \in S^{k-1}$ (see [3, Section 7.5] for more details).

2.9. Jacobians of Sobolev maps. We recall here some basic facts about Jacobians of Sobolev maps; see $[3,28]$ for further details and additional references. Let $y=\left(y_{1}, \ldots, y_{k}\right)$ denote the variable in $\mathbb{R}^{k}$, and $\left\{d y_{1}, \ldots, d y_{k}\right\}$ the standard basis of $\wedge^{1}\left(\mathbb{R}^{k}\right)$. Then the wedge product $d y:=d y_{1} \wedge \cdots \wedge d y_{k}$ is the standard volume-form on $\mathbb{R}^{k}$, and $k \cdot d y$ is the differential of the $(k-1)$-form

$$
\omega_{0}(y):=\sum_{i}(-1)^{i-1} y_{i} \widehat{d y_{i}},
$$

where $\widehat{d y_{i}}$ stands for the wedge product of all $d y_{j}$ with $j \neq i$. The $k$-dimensional Jacobian of a map $u \in W^{1, k}\left(\Omega, \mathbb{R}^{k}\right)$ is the pull-back of $d y$ according to $u$, namely the $k$-form

$$
J u:=d u_{1} \wedge \cdots \wedge d u_{k}=u^{\sharp}(d y)=\frac{1}{k} d\left(u^{\sharp} \omega_{0}\right),
$$

where $d u_{i}=\sum_{j} D_{j} u_{i} d x_{j}$ is the differential of the $i$-th component of $u . J$ is a continuous operator from $W^{1, k}\left(\Omega, \mathbb{R}^{k}\right)$ into $L^{1}\left(\Omega, \Lambda^{k}\left(\mathbb{R}^{n+k}\right)\right)$.

2.10. Jacobians as currents. Let $\left\{e_{1}, \ldots, e_{n+k}\right\}$ be the standard basis of $\mathbb{R}^{n+k}$. We identify vector and covectors on $\mathbb{R}^{n+k}$ using the operator $\star$ defined in [3, Section 2.7]: given a covector $\alpha \in \Lambda^{h}\left(\mathbb{R}^{n+k}\right)$, the vector $\star \alpha \in \Lambda_{n+k-h}\left(\mathbb{R}^{n+k}\right)$ is uniquely determined by the identity

$$
\alpha^{\prime} \cdot(\star \alpha)=\left(\alpha^{\prime} \wedge \alpha\right) \cdot\left(e_{1} \wedge \cdots \wedge e_{n+k}\right), \quad \text { for every } \alpha^{\prime} \in \bigwedge^{n+k-h}\left(\mathbb{R}^{n+k}\right) .
$$

Thus, given an $h$-form $\omega$ in $L_{\text {loc }}^{1}(\Omega), \star \omega$ is the $(n+k-h)$-current on $\Omega$ defined by

$$
\star \omega\left[\omega^{\prime}\right]:=\int_{\Omega}\left(\omega^{\prime} \wedge \omega\right) \cdot \tau_{\Omega}, \quad \text { for every } \omega^{\prime} \in \mathcal{D}^{n+k-h}(\Omega),
$$

where $\tau_{\Omega}:=e_{1} \wedge \cdots \wedge e_{n+k}$ is the standard orientation of $\Omega$. There holds

$$
\partial(\star \omega)=(-1)^{n+k-h} \star(d \omega) .
$$

Given a map $u \in W^{1, k}\left(\Omega, \mathbb{R}^{k}\right), \star J u$ is an $n$-current in $L^{1}(\Omega)$, and since $J u$ is a differential (cf. (2.8)), then $\star J u$ is a boundary. For $n=0$, the 0 -current $\star J u$ is the real function $\operatorname{det}(D u)$; for $k=2$ and $n=1$, the 1 -current $\star J u$ is the vector product $D u_{1} \times D u_{2}$. 
Remark 2.11. Unlike the wedge product $d u_{1} \wedge \cdots \wedge d u_{k}$, the last term in line (2.8) makes sense, at least as a distribution, also for maps $u$ in $L^{\infty} \cap$ $W^{1, k-1}\left(\Omega, \mathbb{R}^{k}\right)$, and is therefore taken as a definition of the Jacobian in this case (cf. $[3,28]$ ). In this case $\star J u$ may have infinite mass, but still $\mathbf{F}_{\Omega}(\star J u) \leq$ $\|u\|_{\infty}\|D u\|_{k-1}^{k-1}$. Hence, for every closed bounded set $E \subset \mathbb{R}^{k}, \star J$ is a continuous operator from $W^{1, k-1}(\Omega, E)$ into the space of $n$-dimensional boundaries in $\Omega$ with finite flat norm $\mathbf{F}_{\Omega}$.

\section{Proof of Statement (i) of Theorem $\mathbf{1 . 1}$}

The main step is the following proposition.

Proposition 3.1. Assume a (countable) sequence of smooth maps $\left(u_{\varepsilon}\right)$ on $\Omega$ such that $F_{\varepsilon}\left(u_{\varepsilon}\right)=O(|\log \varepsilon|)$. Then, for every open set $U \Subset \Omega$, we can extract a subsequence (not relabelled) such that $\mathbf{F}_{U}\left(\star J u_{\varepsilon}-\alpha_{k} M\right) \rightarrow 0$, where $M$ is an integral boundary with finite mass in $U$. Moreover, for every simple $n$-covector $\eta$ such that $|\eta|=1$ there holds

$$
\liminf _{\varepsilon \rightarrow 0} \frac{1}{|\log \varepsilon|} F_{\varepsilon}\left(u_{\varepsilon}\right) \geq \beta_{k}|\eta \cdot M|(U) .
$$

Here $M$ is viewed as a vector measure on $U$ and $\eta \cdot M$ is the real measure defined by $(\eta \cdot M)(B):=\eta \cdot(M(B))$ for every set $B \subset U$.

The proof of this statement is based on a variant of the polyhedral deformation theorem. To this purpose, we need some additional notation.

3.2. Grids. Given $\ell>0$ and $a \in \mathbb{R}^{n+k}$, we call grid of size $\ell$ and center $a$ the collection $\mathcal{G}=\mathcal{G}(\ell, a)$ of all closed cubes of the form $Q=a+\ell z+[0, \ell]^{n+k}$ such that $z$ has integral coordinates; $\mathcal{G}^{\prime}=\mathcal{G}^{\prime}(\ell, a)$ is the dual grid associated to $\mathcal{G}$, and precisely $\mathcal{G}:=\mathcal{G}\left(\ell, a^{\prime}\right)$ with $a^{\prime}:=a+(\ell / 2, \ldots, \ell / 2)$. Given $h=0,1, \ldots$, $n+k$, we call $h$-cells (of the grid) the $h$-dimensional faces of the cubes in the grid. For every $h$-cell $Q$ we denote by $Q^{\prime}$ the unique $(n+k-h)$-cell in the dual grid which intersects $Q$. Hence $Q$ and $Q^{\prime}$ lie on orthogonal affine spaces which intersect at the center of $Q$ (see Figure 3.1 below).

We denote by $R_{h}=R_{h}(\ell, a)$ the $h$-skeleton of $\mathcal{G}$, that is, the union of all $h$-cells in the grid $\mathcal{G}$, and by $R_{h}^{\prime}=R_{h}^{\prime}(\ell, a)$ the $h$-skeleton of the dual grid. In the following, we consider sequences of grids $\mathcal{G}_{\varepsilon}=\mathcal{G}(\ell(\varepsilon), a(\varepsilon))$. In this case $\mathcal{G}_{\varepsilon}^{\prime}$, $R_{\varepsilon, h}, R_{\varepsilon, h}^{\prime}$, etc., denote the obvious objects.

3.3. Structure of the proof of Proposition 3.1. We first notice that if the grids $\mathcal{G}_{\varepsilon}$ satisfy certain assumptions, then $\left|u_{\varepsilon}\right| \geq \frac{1}{2}$ on the $(k-1)$-skeleton $R_{\varepsilon, k-1}$ for all $\varepsilon$ sufficiently small. We then introduce a modified Jacobian operator $J_{\psi}$ with the following properties: the sequences $\left(\star J u_{\varepsilon}\right)$ and $\left(\star J_{\psi} u_{\varepsilon}\right)$ are asymptotically equivalent in the flat norm $\mathbf{F}_{\Omega}$, and $J_{\psi} u_{\varepsilon}$ is supported away from $R_{\varepsilon, k-1}$. 


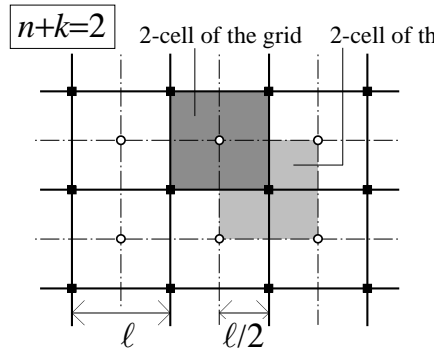

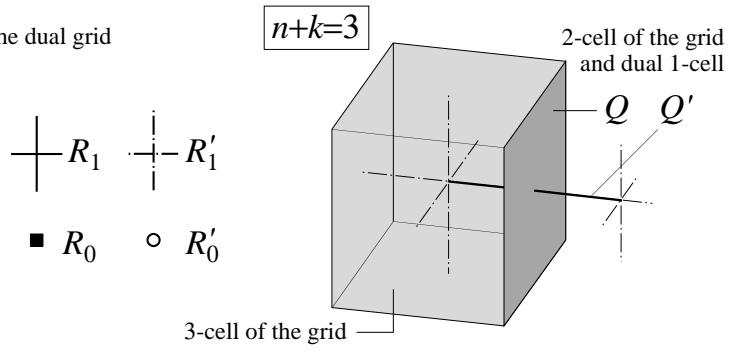

FIGURE 3.1 .

In the next step, we project $\star J_{\psi} u_{\varepsilon}$ on the dual $n$-skeleton $R_{\varepsilon, n}^{\prime}$ using a suitable retraction of the complement of $R_{\varepsilon, k-1}$ onto $R_{\varepsilon, n}^{\prime}$, and show that the multiplicity of the projected current on each dual $n$-cell $Q^{\prime}$ is equal to $\alpha_{k} d_{Q}$, where $d_{Q}$ is the degree of the restriction of $u_{\varepsilon} /\left|u_{\varepsilon}\right|$ to the boundary of the $k$-cell $Q$. In particular the projected currents can be written as $\alpha_{k} M_{\varepsilon}$ for suitable integral currents $M_{\varepsilon}$. Moreover, if certain additional assumptions are satisfied, then the sequences $\left(\star J_{\psi} u_{\varepsilon}\right)$ and $\left(\alpha_{k} M_{\varepsilon}\right)$ are asymptotically equivalent in the flat norm $\mathbf{F}_{U}$, and the masses $\left\|M_{\varepsilon}\right\|_{U}$ can be efficiently estimated in terms of the energies $F_{\varepsilon}\left(u_{\varepsilon}\right)$.

Thus the currents $M_{\varepsilon}$, and consequently the Jacobians $\star J u_{\varepsilon}$, admit a converging subsequence by the closure theorem for integral currents. We conclude the proof by showing that the grids $\mathcal{G}_{\varepsilon}$ can be chosen so that all assumptions made in the previous steps are satisfied.

We first gather some preliminary results (Lemmas 3.4-3.11) and then prove Proposition 3.1.

Lemma 3.4. Let $\mathcal{G}_{\varepsilon}$ be a (countable) sequence of grids with size $\ell=\ell(\varepsilon)$, and denote by $R_{\varepsilon, k-1}(\Omega)$ the union of all $(k-1)$-cells in $R_{\varepsilon, k-1}$ which are contained in $\Omega$. Assume smooth maps $u_{\varepsilon}: \Omega \rightarrow \mathbb{R}^{k}$ such that, for some $b<2 / k$,

$$
\ell^{n+1} \int_{R_{\varepsilon, k-1}(\Omega)} e_{\varepsilon}\left(u_{\varepsilon}\right) d \mathcal{H}^{k-1}=O\left(\varepsilon^{-b}\right) .
$$

If $\ell$ tends to 0 sufficiently slow, and more precisely if $\varepsilon^{c}=O(\ell(\varepsilon))$ for some positive $c<(2 / k-b) /(n+1)$, then the functions $\left|u_{\varepsilon}\right|$ converge to 1 uniformly on $R_{\varepsilon, k-1}(\Omega)$ as $\varepsilon \rightarrow 0$. In particular, $\left|u_{\varepsilon}\right| \geq \frac{1}{2}$ on $R_{\varepsilon, k-1}(\Omega)$ for $\varepsilon$ sufficiently small.

Remark. It can be easily shown (cf. Lemma 3.11) that condition (3.2) is verified if $F_{\varepsilon}\left(u_{\varepsilon}, \Omega\right)=O\left(\varepsilon^{-b}\right)$ and the centers of the grids $\mathcal{G}_{\varepsilon}$ are properly chosen.

Proof. Let $v_{\varepsilon}:=\left|u_{\varepsilon}\right|$ and $R_{\varepsilon}:=R_{\varepsilon, k-1}(\Omega)$. To prove the claim we will show that the oscillations of $v_{\varepsilon}$ over each $(k-1)$-cell of $R_{\varepsilon}$ tend uniformly to 0 as $\varepsilon \rightarrow 0$, while the averages of $v_{\varepsilon}$ tend uniformly to 1 . 
Fix for the time being $\lambda \in(0,1)$. Take a positive continuous function $\varphi$ on $[0,+\infty)$ which vanishes only in 1 and satisfies $\varphi(|y|) \leq W(y)$ for every $y \in \mathbb{R}^{k}$, and denote by $\Phi$ a primitive of $\varphi^{\lambda /((1-\lambda) k)}$.

To begin with, we denote by $g(\varepsilon)$ the left-hand side of (3.2). Then ${ }^{12}$

$$
\begin{aligned}
g(\varepsilon) & \geq \ell^{n+1} \int_{R_{\varepsilon}} \frac{1}{k}\left|D v_{\varepsilon}\right|^{k}+\frac{1}{\varepsilon^{2}} \varphi\left(v_{\varepsilon}\right) d \mathcal{H}^{k-1} \\
& \geq K \ell^{n+1} \int_{R_{\varepsilon}}\left|D v_{\varepsilon}\right|^{(1-\lambda) k} \varepsilon^{-2 \lambda} \varphi^{\lambda}\left(v_{\varepsilon}\right) d \mathcal{H}^{k-1} \\
& \geq K \ell^{n+1} \varepsilon^{-2 \lambda} \int_{R_{\varepsilon}}\left|D\left(\Phi\left(v_{\varepsilon}\right)\right)\right|^{(1-\lambda) k} d \mathcal{H}^{k-1} .
\end{aligned}
$$

Now we use (3.3) and the Sobolev-Morrey embedding theorem to estimate the oscillation of $\Phi\left(v_{\varepsilon}\right)$ on each $(k-1)$-cell $Q$ of $R_{\varepsilon}$. To this end we need that $(1-\lambda) k>k-1$, that is, $\lambda<1 / k$ :

$$
\begin{aligned}
{\left[\operatorname{osc}\left(\Phi\left(v_{\varepsilon}\right), Q\right)\right]^{(1-\lambda) k} } & \leq K \ell^{1-\lambda k} \int_{Q}\left|D\left(\Phi\left(v_{\varepsilon}\right)\right)\right|^{(1-\lambda) k} d \mathcal{H}^{k-1} \\
& \leq K \ell^{-(n+\lambda k)} \varepsilon^{2 \lambda} g(\varepsilon)=O\left(\varepsilon^{-(n+\lambda k) c+2 \lambda-b}\right) .
\end{aligned}
$$

Hence these oscillations converge uniformly to 0 if $-(n+\lambda k) c+2 \lambda-b>0$, i.e.,

$$
c<\frac{2 \lambda-b}{n+\lambda k}
$$

and because of the assumption on $c$, it is clearly possible to choose $\lambda<1 / k$ so that this inequality holds. Moreover, since $\Phi$ is continuous and strictly increasing, also the oscillations of $v_{\varepsilon}$ on each ( $\left.k-1\right)$-cell $Q$ of $R_{\varepsilon}$ converge uniformly to 0 .

Now we use (3.2) to estimate the average of $\varphi\left(v_{\varepsilon}\right)$ over each cell

$$
\frac{1}{\ell^{k-1}} \int_{Q} \varphi\left(v_{\varepsilon}\right) d \mathcal{H}^{k-1} \leq \frac{1}{\ell^{k-1}} \int_{R_{\varepsilon}} W\left(u_{\varepsilon}\right) d \mathcal{H}^{k-1} \leq O\left(\varepsilon^{-(n+k) c+2-b}\right) .
$$

As the assumption on $c$ implies $-(n+k) c+2-b>0$, these averages converge uniformly to 0 . Moreover, since $\varphi$ is continuous, vanishes in 1 and is strictly positive elsewhere, then the averages of $v_{\varepsilon}$ converge uniformly to 1 .

3.5. Modified Jacobians. Let a bounded continuous $k$-form $\psi$ on $\mathbb{R}^{k}$ be given. For every function $u \in W^{1, k}\left(\Omega, \mathbb{R}^{k}\right)$, we denote by $J_{\psi} u$ the pull-back of $\psi$ according to $u$. In other words, writing $\psi=\rho d y$ with $\rho$ a bounded continuous function on $\mathbb{R}^{k}$,

$$
J_{\psi} u:=u^{\sharp} \psi=u^{\sharp}(\rho d y)=\rho(u) J u .
$$

\footnotetext{
${ }^{12}$ In the first inequality we use that $\left|D u_{\varepsilon}\right| \geq\left|D v_{\varepsilon}\right|$ and $W\left(u_{\varepsilon}\right) \geq \varphi\left(v_{\varepsilon}\right)$, while the second one follows from Young's inequality $a+b \geq K a^{1-\lambda} b^{\lambda}$ applied with $a:=\left|D v_{\varepsilon}\right|^{k}$ and $b:=\varepsilon^{-2} \varphi\left(v_{\varepsilon}\right)$.
} 
We show below (Lemma 3.7) that, when the integral of $\rho$ over the unit ball $B_{1}^{k}$ is $\alpha_{k}$, the sequences $\star J u_{\varepsilon}$ and $\star J_{\psi} u_{\varepsilon}$ are asymptotically equivalent in the flat norm $\mathbf{F}_{\Omega}$ provided that $F_{\varepsilon}\left(u_{\varepsilon}\right)=o\left(\varepsilon^{-2 / k}\right)$. This will turn quite useful when proving the compactness part of statement (i) of Theorem 1.1.

Lemma 3.6. Let $\rho: \mathbb{R}^{k} \rightarrow \mathbb{R}$ be a bounded function of class $C^{1}$ such that

$$
\int_{B_{1}^{k}} \rho(y) d y=\alpha_{k}
$$

Then there exists a $(k-1)$-form $\omega$ on $\mathbb{R}^{k}$ of class $C^{1}$ such that $d \omega=(1-\rho) d y$, $\omega(y)=0$ for every $y \in S^{k-1}$, and $\omega$ has growth at most linear at infinity.

Proof. If we write $\omega=\sum(-1)^{i-1} a_{i} \widehat{d y}_{i}$ (cf. Section 2.9), then the problem reduces to finding a bounded $C^{1}$ vectorfield $a=\left(a_{1}, \ldots, a_{k}\right)$ on $\mathbb{R}^{k}$ which is null on $S^{k-1}$ and solves $\operatorname{div} a=f$ on $\mathbb{R}^{k}$, where $f:=1-\rho$.

By assumption (3.5), the integral of $f$ on $B_{1}^{k}$ is 0 . Using this fact we can decompose $f$ as $f=f_{1}+f_{2}$ where $f_{1}$ satisfies

$$
\int_{0}^{1} f_{1}(t y) t^{k-1} d t=0 \quad \text { for every } y \in S^{k-1},
$$

and $f_{2}$ is of the form $f_{2}(y)=h_{1}(|y|) \cdot h_{2}(y /|y|)$ with $h_{1}$ a non-negative $C^{1}$ function on $\mathbb{R}$ with support in $(0,1)$ and integral equal to 1 , and $h_{2}$ a $C^{1}$ function on $S^{k-1}$ with integral equal to 0 . To obtain such a decomposition, one can choose $h_{1}$ arbitrarily, set

$$
h_{2}(y):=\int_{0}^{1} f(t y) t^{k-1} d t,
$$

and then take $f_{1}:=f-f_{2}$.

We first look for a solution of $\operatorname{div} a_{1}=f_{1}$ of the form $a_{1}=g(y) y$ with $g$ a real function on $\mathbb{R}^{k}$. Then the equation reduces to $y \cdot D g+k g=f_{1}$, and the theory of characteristics provides the explicit solution

$$
g(y):=\int_{0}^{1} f_{1}(t y) t^{k-1} d t .
$$

The function $g$ is of class $C^{1}$ and bounded because so is $f_{1}$, and (3.6) implies $g(y)=0$ for every $y \in S^{k-1}$. Hence $a_{1}$ is of class $C^{1}$, grows at most linearly at infinity, and is null on $S^{k-1}$.

Since the integral of the function $h_{2}$ on $S^{k-1}$ must be 0 , and $S^{k-1}$ is a connected manifold, there exists a $C^{1}$ vectorfield $b$ on $S^{k-1}$ whose divergence is $h_{2}$ (one can take the gradient of the solution of the Laplace equation $\Delta u=h_{2}$, or exploit the fact that the $(k-1)$-form $h_{2} \omega_{0}$, with $\omega_{0}$ the standard volume form 
on $S^{k-1}$, has integral 0 on $S^{k-1}$ and therefore is a differential-cf. [12, Corollary 5.8]). Now it is easy to check that the vectorfield $a_{2}(y):=|y| h_{1}(|y|) b(y /|y|)$ solves $\operatorname{div} a_{2}=f_{2}$, is of class $C^{1}$, and $\operatorname{supp}\left(a_{2}\right) \subset B_{1}^{k}$.

Finally, we take $a:=a_{1}+a_{2}$.

Lemma 3.7. Let $\psi:=\rho d y$ with $\rho: \mathbb{R}^{k} \rightarrow \mathbb{R}$ a bounded function of class $C^{1}$ such that (3.5) holds. For every $u \in W^{1, k}\left(\Omega, \mathbb{R}^{k}\right)$ and $\varepsilon>0$ there holds

$$
\mathbf{F}_{\Omega}\left(\star J u-\star J_{\psi} u\right) \leq C \varepsilon^{2 / k} F_{\varepsilon}(u),
$$

where $C$ is a constant that depends only on the choice of $\rho$.

Proof. Take $\omega$ as in Lemma 3.6. Taking formula (2.10) into account we obtain

$$
\star J u-\star J_{\psi} u=\star u^{\sharp}((1-\rho) d y)=\star u^{\sharp}(d \omega)=(-1)^{n} \partial\left(\star u^{\sharp} \omega\right),
$$

and by the definition of flat norm,

$$
\mathbf{F}_{\Omega}\left(\star J u-\star J_{\psi} u\right) \leq\left\|\star u^{\sharp} \omega\right\| \leq \int_{\Omega}|\omega(u)| \cdot|D u|^{k-1} .
$$

The form $\omega$ grows at most linearly at infinity, and since it is of class $C^{1}$ and vanishes on $S^{k-1}$, then it grows at most linearly around $S^{k-1}$, too. On the other hand, the potential $W$ has growth of order at least $k$ at infinity and around $S^{k-1}$, and therefore there exists a constant $C$, depending on $\rho$ only, such that

$$
|\omega(y)| \leq C W^{1 / k}(y) .
$$

To conclude the proof, it is enough to combine estimates (3.8) and (3.9):

$$
\mathbf{F}_{\Omega}\left(\star J u-\star J_{\psi} u\right) \leq C \int_{\Omega} W^{1 / k}(u) \cdot|D u|^{k-1} \leq C \varepsilon^{2 / k} \int_{\Omega}|D u|^{k}+\frac{1}{\varepsilon^{2}} W(u),
$$

where the last inequality follows by applying Young's inequality $a b \leq a^{k}+b^{k /(k-1)}$ with $a:=\varepsilon^{-2 / k} W^{1 / k}(u)$ and $b:=|D u|^{k-1}$.

The next lemma contains a version (in fact, a simplified one) of Federer-Fleming deformation theorem. Since none of the statements is exactly included in the standard formulations of this theorem (see for instance [39, Section 29], [20, Section 4.2]) we have included a detailed sketch of the proof.

Lemma 3.8. Let a grid $\mathcal{G}=\mathcal{G}(\ell, a)$ and a bounded open set $V$ in $\mathbb{R}^{n+k}$ be given. Denote by $d:=2 \sqrt{n+k} \ell$ the double of the diameter of the cubes in $\mathcal{G}$, and by $V_{d}$ the closed d-neighbourhood of $V$. The following statements hold. 
(i) There exists a map $\Phi: \mathbb{R}^{n+k} \backslash R_{k-1} \rightarrow R_{n}^{\prime}$ which is locally Lipschitz, maps every cube in the grid $\mathcal{G}$ into itself, and satisfies

$$
|D \Phi(x)| \leq K \ell / \operatorname{dist}\left(x, R_{k-1}\right) \quad \text { for every } x \in \mathbb{R}^{n+k} \backslash R_{k-1} .
$$

(ii) Let $T$ be an $n$-dimensional current in $\mathbb{R}^{n+k}$ with finite mass and compact support such that $\operatorname{supp}(T) \cap R_{k-1}=\varnothing$. The push-forward $\Phi_{\sharp} T$ is a welldefined current with finite mass in $\mathbb{R}^{n+k}$ supported on $R_{n}^{\prime}$. If in addition $\operatorname{supp}(\partial T) \cap V_{d}=\varnothing$, then the restriction of $\Phi_{\sharp} T$ to $V$ is a real polyhedral current without boundary, and

$$
\mathbf{F}_{V}\left(T-\Phi_{\#} T\right) \leq K \ell^{n+1} \int_{V_{d}} \frac{d|T|(x)}{\left[\operatorname{dist}\left(x, R_{k-1}\right)\right]^{n}} .
$$

(iii) Let $M$ be a compact smooth $n$-surface in $\mathbb{R}^{n+k}$ such that $\operatorname{supp}(M) \cap R_{k-1}=\varnothing$ and $\operatorname{supp}(\partial M) \cap V_{d}=\varnothing$, and let $Q$ be a $k$-cell in the grid whose dual $n$-cell $Q^{\prime}$ intersects $V$. If $M$ is transversal to $Q$, then the multiplicity of $\Phi_{\sharp} M$ on $Q^{\prime}$ is constant and agrees, up to a sign, with the intersection number of $M$ and $Q$ (namely, the sum over all $x \in M \cap Q$ of +1 if $\tau_{M}(x) \wedge \tau_{Q}(x)$ agrees with the standard orientation of $\mathbb{R}^{n+k}$, and -1 otherwise).

Proof. First we construct the map $\Phi$ on the convex hull $H_{Q}$ of $Q \cup Q^{\prime}$, where $Q$ is a $k$-cell in the grid and $Q^{\prime}$ the associated dual cell. We write $x \in \mathbb{R}^{n+k}$ as $\left(s^{\prime}, s\right) \in \mathbb{R}^{n} \times \mathbb{R}^{k}$. We can safely assume that $Q$ is the set of all $\left(s^{\prime}, s\right)$ such that $s^{\prime}=0$ and $|s|_{\infty} \leq \ell / 2$, where $|s|_{\infty}:=\max _{i}\left|s_{i}\right|$, and $Q^{\prime}$ is the set of all $\left(s^{\prime}, s\right)$ such that $s=0$ and $\left|s^{\prime}\right|_{\infty} \leq \ell / 2$ (see Figure 3.2). Then $H_{Q}$ is given by $\left|s^{\prime}\right|_{\infty}+|s|_{\infty} \leq \ell / 2$. We define $\Phi_{Q}: H_{Q} \backslash \partial Q \rightarrow Q^{\prime}$ by

$$
\Phi_{Q}\left(s^{\prime}, s\right):=\left(\frac{\ell s^{\prime}}{\ell-2|s|_{\infty}}, 0\right) \text {. }
$$

One verifies that $\Phi_{Q}$ is singular on $\partial Q$, and satisfies $\left|D \Phi_{Q}(x)\right| \leq K \ell / \operatorname{dist}(x, \partial Q)$.

The hulls $H_{Q}$, with $Q$ ranging among all $k$-cells in the grid, cover $\mathbb{R}^{n+k}$ and have pairwise disjoint interiors, and the associated maps $\Phi_{Q}$ agree on their common faces. Therefore, to conclude the proof of statement (i), it suffices to take $\Phi$ equal to $\Phi_{Q}$ on each $H_{Q}$.

The proof of statement (ii) is standard. The push-forward $\Phi_{\sharp} T$ is a welldefined current because $T$ is supported away from the singular set of $\Phi$. Denote by $V^{\prime}$ the interior of the union of all closed cubes in the grid $\mathcal{G}$ that are contained in $V_{d}$. Thus $V_{d} \supset V^{\prime} \supset V$. We set $\Psi(t, x):=(1-t) x+t \Phi(x)$ for all $t \in \mathbb{R}$, $x \in \mathbb{R}^{n+k} \backslash R_{k-1}$, and define

$$
S:=\Psi_{\sharp}(I \times T),
$$



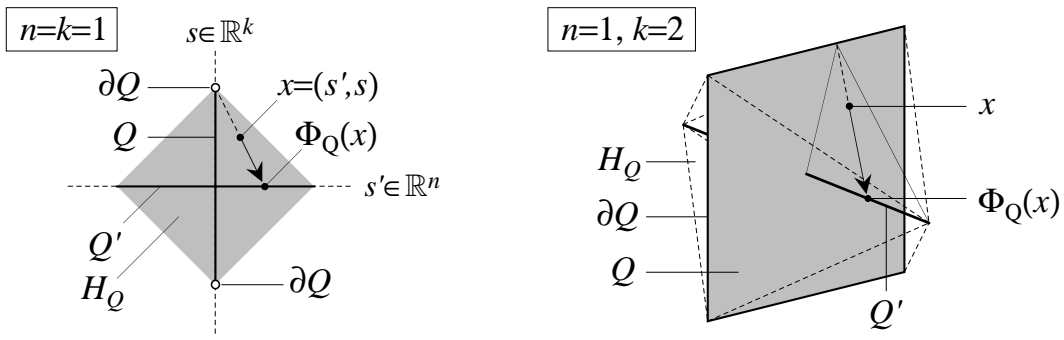

\section{FIGURE 3.2.}

where $I$ is the 1 -current associated to the oriented segment $[0,1]$. Since $\Psi(0, x)=$ $x$ and $\Psi(1, x)=\Phi(x)$, if we assume that $\operatorname{supp}(\partial T) \cap V_{d}=\varnothing$, then ${ }^{13}$

$$
\partial S=\Phi_{\sharp} T-T \text { in } V^{\prime} .
$$

In particular, since $T$ has no boundary in $V^{\prime}$, the same holds for $\Phi_{\sharp} T$. Hence the multiplicity of $\Phi_{\sharp} T$ on each $n$-cell of $R_{n}^{\prime}$ contained in $V^{\prime}$ is constant (and therefore $\Phi_{\sharp} T$ agrees in $V^{\prime}$ with a real polyhedral current). holds

Given a unit vector $e_{0}$ in $\mathbb{R} \times\{0\}$ and a unit $n$-vector $\tau$ in $\{0\} \times \mathbb{R}^{n+k}$, there

$$
\begin{aligned}
\left|[D \Psi(t, x)]_{\sharp}\left(e_{0} \wedge \tau\right)\right| & \leq\left|D_{t} \Psi(t, x)\right| \cdot\left|D_{x} \Psi(t, x)\right|^{n} \\
& \leq|x-\Phi(x)| \cdot|D \Phi(x)|^{n} \leq K \ell^{n+1} \cdot\left[\operatorname{dist}\left(x, R_{k-1}\right)\right]^{-n},
\end{aligned}
$$

and integrating this inequality with respect to the mass of the current $I \times T$ namely the measure $\mathcal{L}^{1}\llcorner[0,1] \times|T|$ — we obtain

$$
\|S\|_{V^{\prime}}=\left\|\Psi_{\#}(I \times T)\right\|_{V^{\prime}} \leq K \ell^{n+1} \int_{\bar{V}^{\prime}} \frac{d|T|(x)}{\left[\operatorname{dist}\left(x, R_{k-1}\right)\right]^{n}},
$$

which, in view of (3.12) and of the inclusions $V \subset V^{\prime}$ and $\bar{V}^{\prime} \subset V_{d}$, implies (3.11).

It remains to prove statement (iii). Since $Q^{\prime}$ intersects $V$, then $Q$ and $Q^{\prime}$ are contained in $V^{\prime}$, and, as pointed out before, the multiplicity of $\Phi_{\sharp} M$ on $Q^{\prime}$ is constant. Moreover, if $x$ is the intersection point of $Q$ and $Q^{\prime}$, then $\Phi^{-1}(x)=Q$ (cf. Figure 3.2), and therefore the multiplicity of $\Phi_{\sharp} M$ at $x$ is the intersection number of $M$ and $Q$ (up to a sign which depends on the orientations of $Q$ and $Q^{\prime}$, which have not been fixed).

${ }^{13}$ As $I \times T$ is supported away from the singular set of $\Psi$, the push-forward $S$ is well defined and $\partial S=\Psi_{\#}(\partial I \times T-I \times \partial T)$. Since $\Phi$ maps each cube $Q$ in the grid into itself, then $\Psi$ maps $[0,1] \times Q$ into $Q$, and the assumption $\operatorname{supp}(\partial T) \cap V_{d}=\varnothing$ implies $\Psi$ maps $[0,1] \times \operatorname{supp}(\partial T)$ in the complement of $V^{\prime}$. Therefore, inside $V^{\prime}$ there holds $\partial S=\Psi_{\sharp}(\partial(I \times T))=\Psi_{\sharp}\left(\delta_{1} \times T-\delta_{0} \times T\right)=\Phi_{\sharp} T-T$. 
The next statement is an almost straightforward application of the previous lemma to Jacobians. $\mathcal{G}, \Phi, V, d$, and $V_{d}$ are taken as in Lemma 3.8, while $\psi=\rho d y$ and $J_{\psi} u$ are taken as in Section 3.5.

Lemma 3.9. Take $0<t<1$, and assume that $\psi=\rho d y$ is supported in the ball $B_{t}^{k}$ and has integral $\alpha_{k}$. Let $V^{\prime}$ be a neighbourhood of $V_{d}$ and $u: V^{\prime} \rightarrow \mathbb{R}^{k} a$ map, smooth up to the boundary, such that $|u| \geq t$ on $R_{k-1}$. Let $Q$ be a $k$-cell in the grid $\mathcal{G}$ whose dual $n$-cell $Q^{\prime}$ intersects $V$. The following statements hold.

(i) If $|y|<t$ and $M_{y}$ is a regular level set of $u$, the push-forward $\Phi_{\sharp} M_{y}$ defines an integral polyhedral current in $V$ whose multiplicity on $Q^{\prime}$ agrees, up to a sign, with $d_{Q}:=\operatorname{deg}\left(u /|u|, \partial Q, S^{k-1}\right)$. In particular $\Phi_{\sharp} M_{y}$ does not depend on the choice of $y$. Moreover,

$$
\left|d_{Q}\right| \leq K \int_{Q}|D u|^{k} d \mathcal{H}^{k} .
$$

(ii) The push-forward $\Phi_{\sharp}\left(\star J_{\psi} u\right)$ is well-defined and agrees with $\alpha_{k} \Phi_{\sharp} M_{y}$ for every regular value $y$ with $|y|<t$. Thus $\Phi_{\#}\left(\star J_{\psi} u\right)$ is a real polyhedral boundary in $V$ with multiplicity $\pm \alpha_{k} d_{Q}$ on $Q^{\prime}$. Moreover,

$$
\mathbf{F}_{V}\left(\star J_{\psi} u-\Phi_{\#}\left(\star J_{\psi} u\right)\right) \leq K\|\psi\|_{\infty} \ell^{n+1} \int_{V_{d}} \frac{|D u(x)|^{k} d x}{\left[\operatorname{dist}\left(x, R_{k-1}\right)\right]^{n}} .
$$

Proof. We prove statement (i) first. By a density argument we can assume that $y$ is a regular value in the classical sense and $M_{y}$ is transversal to $Q$. Since $|y|<t$ and $|u| \geq t$ on $R_{k-1}$, the level set $M_{y}$ is supported away from $R_{k-1}$, and therefore satisfies the assumption of statement (iii) of Lemma 3.8. Hence $\Phi_{\sharp} M_{y}$ agrees with a real polyhedral current on $V$, and its multiplicity on $Q^{\prime}$ is the intersection number of $M_{y}$ and $Q$, which in turn agrees-almost by definitionwith $\operatorname{deg}\left(u, Q, \mathbb{R}^{k}, y\right)$. We claim that

$$
\operatorname{deg}\left(u, Q, \mathbb{R}^{k}, y\right)=d_{Q} .
$$

By integrating identity (3.15) over all $y \in B_{t}^{k}$ we obtain

$$
\alpha_{k} t^{k}\left|d_{Q}\right| \leq \int_{B_{t}^{k}}\left|\operatorname{deg}\left(u, Q, \mathbb{R}^{k}, y\right)\right| d y \leq \int_{Q}|\operatorname{det} D u| \leq \int_{Q}|D u|^{k},
$$

which gives (3.13). It remains to prove (3.15). Consider the truncated map

$$
\tilde{u}(x):= \begin{cases}u(x) & \text { if }|u(x)| \leq t \\ t u(x) /|u(x)| & \text { otherwise. }\end{cases}
$$

Since $|u|>t$ on $R_{k-1}$, which contains $\partial Q$, and $|y|<t$, then $\operatorname{deg}\left(u, Q, \mathbb{R}^{k}, y\right)=$ $\operatorname{deg}\left(\tilde{u}, Q, \mathbb{R}^{k}, y\right)$. Since $\tilde{u}$ maps $\partial Q$ in the sphere of radius $t$, then $\operatorname{deg}\left(\tilde{u}, Q, \mathbb{R}^{k}, y\right)$ 
is equal to $\operatorname{deg}\left(\tilde{u}, \partial Q, S_{t}^{k-1}\right)$ (see, e.g., [3, Section 2.10]), which is clearly the same as $d_{Q}$.

To prove statement (ii), we denote by $T$ the current obtained by extending $\star J_{\psi} u$ to 0 outside $V^{\prime}$. Then $T$ satisfies the assumptions of statement (ii) of Lemma 3.8, and (3.14) follows from (3.11) and the estimate $\left|J_{\psi} u\right| \leq\|\psi\|_{\infty}|D u|^{k}$. Note that $\Phi_{\sharp}\left(\star J_{\psi} u\right)$ is a boundary in $V$ because it has finite flat distance from $\star J_{\psi} u$, which is a boundary in $V$ (see Sections 2.5 and 2.10).

By the coarea formula (see Section 2.7), $\star J_{\psi} u=\rho(u) \star J u$ is given by the integral of the currents $M_{y}$ with respect to the measure $\rho(y) d \mathcal{L}^{k}(y)$. Then, by the linearity of the push-forward operator, $\Phi_{\#}\left(\star J_{\psi} u\right)$ is the integral of the currents $\Phi_{\sharp} M_{y}$. But these currents are all the same for $|y|<t$, and to conclude the proof it suffices to recall that $\rho$ is supported in $B_{t}^{k}$ and its integral is $\alpha_{k}$.

The following key estimate is a direct application of the results in [27].

Lemma 3.10. There exist a universal finite constant $K$ and, for every $r>0$, finite constants $C_{0}$ and $C_{1}$ (depending on $r, k$ and the potential $W$ ) so that, given a cube $Q_{\ell}:=[-\ell / 2, \ell / 2]^{k}$ with $\ell>0$, and a smooth map $u: Q_{\ell} \rightarrow \mathbb{R}^{k}$ such that $|u|>\frac{1}{2}$ on $\partial Q_{\ell}$ and $d:=\operatorname{deg}\left(u /|u|, \partial Q_{\ell}, S^{k-1}\right) \neq 0$, then

$$
\begin{aligned}
\beta_{k}|d| & \cdot\left[\left|\log \left(\varepsilon / \ell^{k / 2}\right)\right|-C_{1}(1+\log |d|)\right] \\
& \leq \int_{Q_{\ell}} e_{\varepsilon}(u) d \mathcal{L}^{k}+K r \ell \int_{\partial Q_{\ell}} e_{\varepsilon}(u) d \mathcal{H}^{k-1}
\end{aligned}
$$

for every $\varepsilon$ such that $\varepsilon / \ell^{k / 2}<1$ and $\left(\varepsilon / \ell^{k / 2}\right) \cdot\left|\log \left(\varepsilon / \ell^{k / 2}\right)\right| \leq C_{0} /|d|$.

Proof. Because of the growth assumptions on the potential $W$ (see Section 2.1), there exists a constant $K$ such that $\left(1-|y|^{2}\right)^{2} \leq K W(y)$. Then it suffices to prove the statement for $W(y)=K^{-1}\left(1-|y|^{2}\right)^{2}$, and by rescaling we can also assume that $K=1$.

It is proved in [27] that for every bounded Lipschitz domain $U \subset \mathbb{R}^{k}$ and $r>0$, there exist constants $C_{0}=C_{0}(U, r)$ and $C_{1}=C_{1}(U, r)$ so that

$$
\beta_{k}|d| \cdot\left[|\log \varepsilon|-C_{1}(1+\log |d|)\right] \leq \int_{U} e_{\varepsilon}(u) d \mathcal{L}^{k},
$$

for every map $u: U \rightarrow \mathbb{R}^{k}$ such that $|u(x)| \geq \frac{1}{2}$ for $\operatorname{dist}(x, \partial U) \leq r$ and every $\varepsilon$ such that $\varepsilon<1$ and $\varepsilon|\log \varepsilon| \leq C_{0} /|d|{ }^{14}$

${ }^{14}$ We have used estimate $\int_{U} e_{\varepsilon}(u) \geq \beta_{k}|d| \cdot \log (\sigma / \varepsilon)-C$ in Theorem 1.2 of [27]. However, the constants $C$ and $\sigma$ given in that statement depend on the domain $U$ and the trace of $u$ on the boundary of $U$. To show that their contribution can be estimated by $C_{1}(1+\log |d|)|d|$ independently of the trace of $u$ and of the degree $d$, one has to go through the proof given in Section 4 of that paper. More precisely, if one rewrites the constant $\sigma$ in Proposition 4.1 as $\sigma_{0} /|d|$, then $\sigma_{0}$ can be arbitrarily chosen in the interval $(0, r / 4)$, independently of $d$, and looking through the proof one finds that the desired inequality holds provided that $\sigma_{0} /|d| \geq s(\varepsilon)$, where $s(\varepsilon)$ is a universal function of order $\varepsilon|\log \varepsilon|$. 
To prove Lemma 3.10 for $\ell=1$, it suffices to apply this result with $U:=$ $Q_{1+r}$ and $u$ extended to $Q_{1+r} \backslash Q_{1}$ by the formula $u(x):=u\left(x /|x|_{\infty}\right)$, where $|x|_{\infty}:=\max \left\{\left|x_{i}\right|\right\}$. Indeed a simple computation gives

$$
\int_{Q_{1+r} \backslash Q_{1}} e_{\varepsilon}(u) d \mathcal{L}^{k} \leq K r \int_{\partial Q_{1}} e_{\varepsilon}(u) d \mathcal{H}^{k-1} .
$$

The statement for general $\ell$ is obtained by scaling.

We finally show that the grid can always be chosen so to satisfy certain additional energy estimates that will be used in the proof of Proposition 3.1.

Lemma 3.11. Assume $u: \Omega \rightarrow \mathbb{R}^{k}$ smooth, and positive numbers $\delta$, $\varepsilon$ and $\ell$. Then there exists $a \in \mathbb{R}^{n+k}$ such that the grid $\mathcal{G}=\mathcal{G}(\ell, a)$ satisfies

$$
\begin{aligned}
\ell^{n} \int_{\widetilde{R}_{k} \cap \Omega} e_{\varepsilon}(u) d \mathcal{H}^{k} & \leq(1+\delta) F_{\varepsilon}(u) \\
\ell^{n} \int_{R_{k} \cap \Omega} e_{\varepsilon}(u) d \mathcal{H}^{k} & \leq \frac{K}{\delta} F_{\varepsilon}(u) \\
\ell^{n+1} \int_{R_{k-1} \cap \Omega} e_{\varepsilon}(u) d \mathcal{H}^{k-1} & \leq \frac{K}{\delta} F_{\varepsilon}(u) \\
\ell^{n} \int_{\Omega} \frac{e_{\varepsilon}(u) d x}{\left[\operatorname{dist}\left(x, R_{k-1}\right)\right]^{n}} & \leq \frac{K}{\delta} F_{\varepsilon}(u),
\end{aligned}
$$

where $\widetilde{R}_{k}$ is the union of all $k$-cells in $R_{k}$ that are parallel to the $k$-plane spanned by $\left\{e_{n+1}, \ldots, e_{n+k}\right\}$.

Proof. Denote the left-hand sides of (3.17), (3.18), (3.19), and (3.20) by $f_{0}(a), f_{1}(a), f_{2}(a)$, and $f_{3}(a)$, respectively. We claim that the average of $f_{0}(a)$ over all $a$ in the cube $Q_{\ell}:=[0, \ell]^{n+k}$ is $F_{\varepsilon}(u)$, while the average of $f_{i}(a)$ agrees with $F_{\varepsilon}(u)$ times some universal constant for $i=1,2,3$. Were this claim true, the rest of the proof would follow by Lemma 8.4 (with $X:=Q_{\ell}$ and $\mu:=\mathcal{L}^{n+k}\left\llcorner Q_{\ell}\right.$ ).

We first compute the average of $f_{0}$. Notice that the set $\widetilde{R}_{k}=\widetilde{R}_{k}(\ell, a)$ depends only on the first $n$-coordinates of $a$ (see Figure 3.3), and then, denoting by $Q_{\ell}^{n}$ the set of all $a \in Q_{\ell}$ whose last $k$-coordinates are null, the average of $f_{0}$ on $Q_{\ell}$ is the same as the average on $Q_{\ell}^{n}$. Hence

$$
f_{Q_{\ell}} f_{0}=\int_{Q_{\ell}^{n}}\left[\int_{\widetilde{R}_{k}(\ell, a) \cap \Omega} e_{\varepsilon}(u) d \mathcal{H}^{k}\right] d \mathcal{L}^{n}(a)=\int_{\Omega} e_{\varepsilon}(u) d \mathcal{L}^{n+k}=F_{\varepsilon}(u),
$$

where the second identity follows by Fubini's theorem (see Figure 3.3).

We compute now the average of $f_{1}$. For every $k$-plane $P$ spanned by subsets of $\left\{e_{1}, \ldots, e_{n+k}\right\}$ we take the union $R_{k}(P)$ of all $k$-cell in the grid that are parallel to $P$, then we proceed as above with $\widetilde{R}_{k}$ replaced by $R_{k}(P)$ and take the sum over all $P$. We thus obtain that the average of $f_{1}$ over $Q_{\ell}$ is $\left(\begin{array}{c}n+k \\ k\end{array}\right)$ times $F_{\varepsilon}(u)$. 


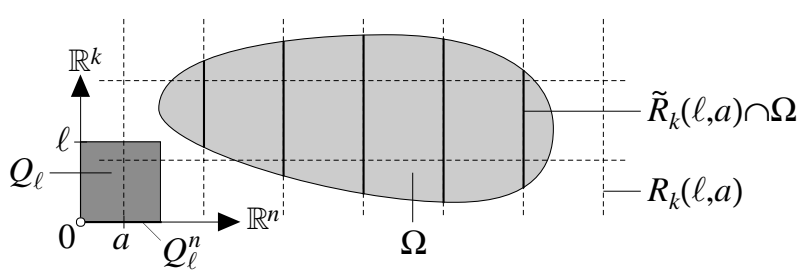

FIGURE 3.3.

In a similar way one proves that the average of $f_{2}$ is $\left(\begin{array}{c}n+k \\ k-1\end{array}\right)$ times $F_{\varepsilon}(u)$.

Finally, we compute the average of $f_{3}$. Let $R$ be the $(k-1)$-skeleton of the grid with size 1 and center 0 . Since $R_{k-1}=a+\ell R$, we have

$$
\operatorname{dist}\left(x, R_{k-1}\right)=\ell \cdot \operatorname{dist}\left(\ell^{-1}(x-a), R\right),
$$

and therefore ${ }^{15}$

$$
\begin{aligned}
f_{Q_{\ell}} f_{3} & =f_{Q_{\ell}}\left[\ell^{n} \int_{\Omega} \frac{e_{\varepsilon}(u) d x}{\left[\ell \operatorname{dist}\left(\ell^{-1}(x-a), R\right)\right]^{n}}\right] d a \\
& =\int_{\Omega}\left[f_{Q_{\ell}} \frac{d a}{\left[\operatorname{dist}\left(\ell^{-1}(x-a), R\right)\right]^{n}}\right] e_{\varepsilon}(u) d x \\
& =\int_{\Omega}\left[f_{Q_{1}} \frac{d y}{[\operatorname{dist}(y, R)]^{n}}\right] e_{\varepsilon}(u) d x .
\end{aligned}
$$

Since the integral between square brackets in the last line of formula (3.21) is finite (Lemma 8.3), the average of $f_{3}$ is $F_{\varepsilon}(u)$ times some universal constant $K$.

Proof of Proposition 3.1 / compactness. Let $u_{\varepsilon}$ be as in the statement of Proposition 3.1, and set $e_{\varepsilon}:=e_{\varepsilon}\left(u_{\varepsilon}\right)$. Passing to a subsequence, we can assume that the liminf at the left-hand side of (3.1) is a limit (and therefore will not be affected by further extractions of subsequences).

We fix a $k$-form $\psi=\rho d y$ on $\mathbb{R}^{k}$ with support contained in $B_{1 / 2}^{k}$ and integral equal to $\alpha_{k}$, and set

$$
\ell=\ell(\varepsilon):=|\log \varepsilon|^{-2} \quad \text { for every } \varepsilon .
$$

We take the grids $\mathcal{G}_{\varepsilon}=\mathcal{G}(a(\varepsilon), \ell(\varepsilon))$, where the centers $a=a(\varepsilon)$ are chosen according to Lemma 3.11, so that (3.17)-(3.20) hold for some positive number $\delta$, with $u$ and $\mathcal{G}$ replaced by $u_{\varepsilon}$ and $\mathcal{G}_{\varepsilon}$. Recall that

$$
F_{\varepsilon}\left(u_{\varepsilon}\right)=O(|\log \varepsilon|) .
$$

${ }^{15}$ The last identity follows from the change of variable $y=\ell^{-1}(x-a)$ and the fact that $Q_{\ell}$ and $Q_{1}$ are periodicity cells for $a \mapsto \operatorname{dist}\left(\ell^{-1}(x-a), R\right)$ and $y \mapsto \operatorname{dist}(y, R)$, respectively. 
We choose two open sets $V$ and $V^{\prime}$ so that $U \Subset V \Subset V^{\prime} \Subset \Omega$. First of all, we notice that Lemma 3.7 and (3.23) yield

$$
\mathbf{F}_{\Omega}\left(\star J u_{\varepsilon}-\star J_{\psi} u_{\varepsilon}\right) \rightarrow 0 .
$$

Lemma 3.4 and estimates (3.19) and (3.23) imply $\left|u_{\varepsilon}\right| \geq \frac{1}{2}$ on $R_{\varepsilon, k-1}(\Omega)$ for $\varepsilon$ sufficiently small.

We want to apply Lemma 3.9 with $u=u_{\varepsilon}, \mathcal{G}=\mathcal{G}_{\varepsilon}$, and $t=\frac{1}{2}$. Since the quantity $d$ which appears in that lemma tends to 0 as $\varepsilon \rightarrow 0$, then $R_{\varepsilon, k-1}(\Omega) \supset$ $R_{\varepsilon, k-1} \cap V_{d}$ and $V^{\prime} \supset V_{d}$ for $\varepsilon$ sufficiently small, and the assumptions of the lemma are satisfied. Hence the projected current $\Phi_{\varepsilon \sharp}\left(\star J_{\psi} u_{\varepsilon}\right)$ can be represented in $V$ as $\alpha_{k} M_{\varepsilon}$, where $M_{\varepsilon}$ is an integral boundary in $V$ supported on $R_{\varepsilon, n}^{\prime}$. Combining estimates (3.14) and $\left|D u_{\varepsilon}\right|^{k} \leq k e_{\varepsilon}$, and recalling (3.20), (3.22), (3.23), we obtain

$$
\mathbf{F}_{V}\left(\star J_{\psi} u_{\varepsilon}-\alpha_{k} M_{\varepsilon}\right)=\ell \cdot O(|\log \varepsilon|) \rightarrow 0 .
$$

Now we estimate the mass of $M_{\varepsilon}$ in $V$. Given a dual $n$-cell $Q^{\prime} \in R_{\varepsilon, k}^{\prime}$ which intersects $V$, the corresponding $k$-cell $Q$ is contained in $\Omega$, and by Lemma 3.9 the multiplicity of $M_{\varepsilon}$ on $Q^{\prime}$ agrees with $d_{Q}:=\operatorname{deg}\left(u_{\varepsilon} /\left|u_{\varepsilon}\right|, \partial Q, S^{k-1}\right)$. Hence

$$
\left\|M_{\varepsilon}\right\|_{V} \leq \ell^{n} \sum_{Q \subset \Omega}\left|d_{Q}\right| .
$$

We fix $r>0$, and use Lemma 3.10 to estimate $\left|d_{Q}\right|$. Since $Q \subset V_{d} \subset \Omega$ for $\varepsilon$ small enough, estimates (3.13) and (3.18) yield

$$
\left|d_{Q}\right| \leq K \int_{Q}|D u|^{k} d \mathcal{H}^{k} \leq K \int_{R_{\varepsilon, k}(\Omega)} e_{\varepsilon} d \mathcal{H}^{k}=O\left(|\log \varepsilon|^{2 n+1}\right) .
$$

Hence $\left(\varepsilon / \ell^{k / 2}\right)\left|\log \left(\varepsilon / \ell^{k / 2}\right)\right| \leq K \varepsilon|\log \varepsilon|^{k+1}=o\left(1 / d_{Q}\right)$, which implies that, for $\varepsilon$ sufficiently small, estimate (3.16) holds for all $Q$. Moreover, by (3.27), the term between square brackets in the first line of (3.16) is asymptotically equivalent to $|\log \varepsilon|$, and therefore (3.16) can be rewritten as

$$
\left(1-c_{r}(\varepsilon)\right) \beta_{k}\left|d_{Q}\right| \leq \frac{1}{|\log \varepsilon|} \int_{Q} e_{\varepsilon} d \mathcal{H}^{k}+\frac{K r \ell}{|\log \varepsilon|} \int_{\partial Q} e_{\varepsilon} d \mathcal{H}^{k-1},
$$

where $c_{r}(\varepsilon)$ tends to 0 as $\varepsilon \rightarrow 0$ (and does not depend on $Q$ ). For $\varepsilon$ sufficiently small $c_{r}(\varepsilon) \leq \frac{1}{2}$, and the previous inequality and (3.26) becomes

$$
\left\|M_{\varepsilon}\right\|_{V} \leq \frac{K \ell^{n}}{|\log \varepsilon|} \int_{R_{\varepsilon, k}(\Omega)} e_{\varepsilon} d \mathcal{H}^{k}+\frac{K \ell^{n+1}}{|\log \varepsilon|} \int_{R_{\varepsilon, k-1}(\Omega)} e_{\varepsilon} d \mathcal{H}^{k-1} .
$$


Estimates (3.29), (3.18), (3.19), and (3.23) imply that the integral boundaries $M_{\varepsilon}$ are uniformly bounded in mass in $V$, and therefore converge in the flat norm $\mathbf{F}_{U}$ to some integral boundary $M$ in $U$ (Proposition 2.6). Finally, (3.25) and (3.24) imply that the currents $\star J u_{\varepsilon}$ and $\star J_{\psi} u_{\varepsilon}$ converge to $\alpha_{k} M$ in the flat norm $\mathbf{F}_{U}$.

Proof of Proposition 3.1 / inequality (3.1). We follow the notation of the previous section. With no loss in generality we may assume $\eta=d x_{1} \wedge \cdots \wedge d x_{n}$. Then the proof of (3.1) just follows from a refinement of estimate (3.29).

Since the current $M_{\varepsilon}$ is supported on the dual $n$-skeleton $R_{\varepsilon, n}^{\prime}$, the positive measure $\left|\eta \cdot M_{\varepsilon}\right|$ is just the part of $\left|M_{\varepsilon}\right|$ supported on the union of the dual $n$-cells $Q^{\prime}$ parallel to the $n$-plane spanned by $\left\{e_{1}, \ldots, e_{n}\right\}$, that is, the duals of the $k$-cells $Q$ contained in $\widetilde{R}_{\varepsilon, k}$ (see Lemma 3.11). Moreover, if $Q^{\prime}$ intersects $V$, then $Q$ is contained in $\Omega$. Hence, taking into account (3.28), (3.17), and (3.19)

$$
\begin{aligned}
\left(1-c_{r}(\varepsilon)\right) \beta_{k}\left|\eta \cdot M_{\varepsilon}\right|(V) & \\
& \leq\left(1-c_{r}(\varepsilon)\right) \beta_{k} \ell^{n} \sum_{Q \subset \widetilde{R}_{\varepsilon, k} \cap \Omega}\left|d_{Q}\right| \\
& \leq \frac{\ell^{n}}{|\log \varepsilon|} \int_{\widetilde{R}_{\varepsilon, k} \cap \Omega} e_{\varepsilon} d \mathcal{H}^{k}+\frac{K r \ell^{n+1}}{|\log \varepsilon|} \int_{R_{\varepsilon, k-1} \cap \Omega} e_{\varepsilon} d \mathcal{H}^{k-1} \\
& \leq \frac{1+\delta}{|\log \varepsilon|} F_{\varepsilon}\left(u_{\varepsilon}\right)+r O(1),
\end{aligned}
$$

and passing to the limit as $\varepsilon \rightarrow 0$,

$$
\beta_{k}|\eta \cdot M|(V) \leq \liminf _{\varepsilon \rightarrow 0} \frac{1+\delta}{|\log \varepsilon|} F_{\varepsilon}\left(u_{\varepsilon}\right)+C r .
$$

Finally, (3.1) follows because $\delta$ and $r$ can be taken arbitrarily small.

Proof of Theorem 1.1 (i). We use the reflection map $\Phi$ described in Remark 8.2 to extend $u_{\varepsilon}$ to $\Omega^{\prime}:=\Omega \cup U$ so that the energy of $u_{\varepsilon}$ on $\Omega^{\prime}$ remains of order $|\log \varepsilon|$. By a density argument, we can also assume that $u_{\varepsilon}$ is smooth in $\Omega^{\prime}$, and then the compactness statement follows from Proposition 3.1 (with $\Omega$ and $U$ replaced by $\Omega^{\prime}$ and $\Omega$, respectively).

We prove the lower-bound inequality (1.3) using a standard localization argument. Given an open set $A \Subset \Omega$ and a simple unit $n$-covector $\eta$, we apply Proposition 3.1 with $\Omega$ replaced by $A$, and taking the supremum in (3.1) over all $U \Subset A$ we obtain

$$
\liminf _{\varepsilon \rightarrow 0} \frac{F_{\varepsilon}\left(u_{\varepsilon}, A\right)}{|\log \varepsilon|} \geq \beta_{k}|\eta \cdot M|(A) .
$$


Now we apply the previous inequality to finitely many pairwise disjoint open sets $A_{i} \Subset \Omega$ and simple unit $n$-covectors $\eta_{i}$, and summing over all $i$ we get

$$
\liminf _{\varepsilon \rightarrow 0} \frac{F_{\varepsilon}\left(u_{\varepsilon}\right)}{|\log \varepsilon|} \geq \beta_{k} \sum_{i}\left|\eta_{i} \cdot M\right|\left(A_{i}\right) .
$$

Finally, taking the supremum over all choices of $A_{i}$ and $\eta_{i}$ we obtain (1.3).

\section{Proof of Statement (ii) of Theorem $\mathbf{1 . 1}$}

The upper bound inequality in Theorem 1.1 is a direct consequence of Theorem 5.10 in [3] and of the construction described in Lemma 4.2 below. We begin with some additional notation.

4.1. Additional notation. Given a polyhedral current $M=\sum_{i} \sigma_{i} F_{i}$, we tacitly assume that the intersection of any two simplexes $F_{i}$ and $F_{j}$ is either empty or a common face of $F_{i}$ and $F_{j}$. With a slight abuse of notation, we use the letter $M$ also to denote the supporting polyhedral set $\bigcup_{i} F_{i}$.

Given an $n$-dimensional simplex $F$ in $\mathbb{R}^{n+k}$ and $\delta, \gamma>0$, let $U=U(F, \delta, \gamma)$ be the set of all $x \in \mathbb{R}^{n+k}$ such that

$$
\operatorname{dist}(x, F) \leq \delta \wedge \frac{\gamma}{\sqrt{1+\gamma^{2}}} \operatorname{dist}(x, \partial F) .
$$

If we identify the $n$-plane spanned by $F$ with $\mathbb{R}^{n}$ and write $x \in \mathbb{R}^{n+k}$ as $x=$ $\left(x^{\prime}, x^{\prime \prime}\right) \in \mathbb{R}^{n} \times \mathbb{R}^{k}$, then $U$ is the set of all $x$ such that (see Figure 4.1 below, or Figure 9.1 in Appendix B)

$$
x^{\prime} \in F \text { and }\left|x^{\prime \prime}\right| \leq g\left(x^{\prime}\right), \quad \text { where } g\left(x^{\prime}\right):=\delta \wedge \gamma \operatorname{dist}\left(x^{\prime}, \partial F\right) .
$$

Jacobians of maps of class $W^{1, k-1}$ valued in the sphere $S^{k-1}$ are intended in the distributional sense (see Remark 2.11).

The next proposition contains the basic construction for the approximating maps $u_{\varepsilon}$. In view of the proof of the upper bound inequality in Theorem 5.5, we have chosen a more general statement than needed for the proof of Theorem 1.1(ii).

Lemma 4.2. Let $N$ be an integral polyhedral current in $\mathbb{R}^{n+k}$ with dimension $n+1$ such that $|\partial N|(\partial \Omega)=0$ and $\partial N$ has multiplicity 1 , and denote by $M$ the restriction of $\partial N$ to $\Omega$. Let these be given: positive numbers $\delta, \gamma$, a map $\tilde{u}: \bar{\Omega} \rightarrow S^{k-1}$, and a finite union $S$ of $(n-1)$-dimensional simplexes in $\mathbb{R}^{n+k}$ which contains all $(n-1)$-dimensional faces of $N$, so that the following holds:

(i) $\tilde{u} \in W^{1, k-1}\left(\Omega, S^{k-1}\right)$ and $\star J \tilde{u}=\alpha_{k} M$;

(ii) $\tilde{u}$ is locally Lipschitz in $\bar{\Omega} \backslash(M \cup S)$ and there exists $p<1+1 / k$ such that

$$
|D \tilde{u}(x)|=O(1 / \operatorname{dist}(x, M))+O\left(1 /(\operatorname{dist}(x, S))^{p}\right) .
$$


(iii) for every $n$-dimensional face $F$ of $\partial N$,

$$
\tilde{u}(x)=x^{\prime \prime} /\left|x^{\prime \prime}\right|, \quad \text { for } x \in U(F, \delta, \gamma) \cap \Omega .
$$

Then the approximating maps $u_{\varepsilon}: \bar{\Omega} \rightarrow \mathbb{R}^{k}$ defined by

$$
u_{\varepsilon}(x):=g_{\varepsilon}(x) \tilde{u}(x), \quad \text { where } g_{\varepsilon}(x):=1 \wedge \frac{\operatorname{dist}(x, M \cup S)}{\varepsilon}
$$

are locally Lipschitz in the complement of $S$, belong to $W^{1, q}\left(\Omega, \mathbb{R}^{k}\right)$ for every $q<$ $(k+1) /(p-1)$, and converge strongly to $\tilde{u}$ in $W^{1, q}(\Omega)$ for every $q<k \wedge(k+1) / p$, and in particular for $q=k-1$. The Jacobians $\star J u_{\varepsilon}$ converge to $\star J \tilde{u}=\alpha_{k} M$ in the flat norm $\mathbf{F}_{\Omega}$, and

$$
\limsup _{\varepsilon \rightarrow 0} \frac{F_{\varepsilon}\left(u_{\varepsilon}\right)}{|\log \varepsilon|} \leq \beta_{k}\|M\|_{\Omega}
$$

Proof. Using Lemma 8.3, the regularity of $u_{\varepsilon}$ and the convergence of $u_{\varepsilon}$ to $\tilde{u}$ are matters of simple computations. The strong convergence in $W^{1, q}$ for $q=k-1$ follows by the assumption on $p$, and implies the convergence of Jacobians (see Remark 2.11). It remains to prove (4.4).
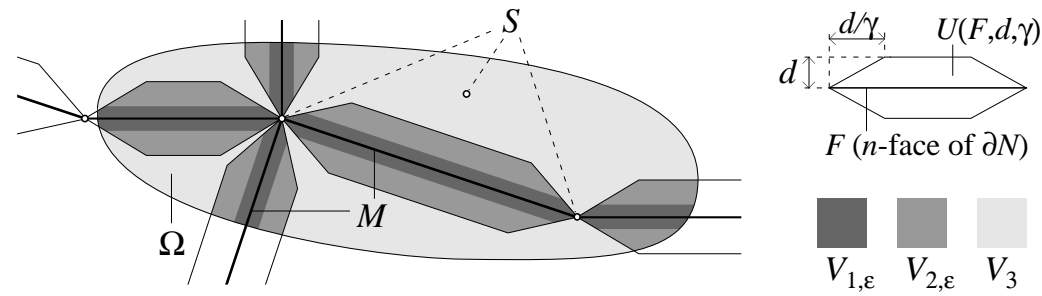

FIGURE 4.1 .

Let $d \in(0, \delta \wedge 1]$ be fixed for the time being, and denote by $U$ the union of the set $U(F, d, \gamma)$ over all $n$-dimensional faces $F$ of $\partial N$. For every $\varepsilon \in(0, d]$ we decompose $\Omega$ as union of the sets $V_{1, \varepsilon}, V_{2, \varepsilon}, V_{3}$ defined as follows (see Figure 4.1):

$$
\begin{aligned}
V_{1, \varepsilon} & :=\{x \in \Omega \cap U: \operatorname{dist}(x, M \cup S) \leq \varepsilon\} \\
V_{2, \varepsilon} & :=(\Omega \cap U) \backslash V_{1, \varepsilon} \\
V_{3} & :=\Omega \backslash U .
\end{aligned}
$$

For the rest of this proof, the letter $C$ denotes any positive finite constant, possibly different at each occurrence, that depends only on the choice of $\Omega, N, \tilde{u}, \delta$ and $\gamma$. 
Since $V_{1, \varepsilon} \subset U$, assumption (iii) implies $|D \tilde{u}(x)| \leq C / \operatorname{dist}(x, M)$, and therefore formula (4.3) yields

$$
\begin{aligned}
\left|D u_{\varepsilon}(x)\right| & \leq g_{\varepsilon}(x) \cdot|D \tilde{u}(x)|+\left|D g_{\varepsilon}(x)\right| \cdot|\tilde{u}(x)| \\
& \leq \frac{C \cdot \operatorname{dist}(x, M \cup S)}{\varepsilon \cdot \operatorname{dist}(x, M)}+\frac{1}{\varepsilon} \leq \frac{C}{\varepsilon} .
\end{aligned}
$$

Using that $W\left(u_{\varepsilon}\right) \leq C$ and $\mathcal{L}^{n+k}\left(V_{1, \varepsilon}\right) \leq C \varepsilon^{k}$ (cf. Lemma 8.3), we obtain

$$
F_{\varepsilon}\left(u_{\varepsilon}, V_{1, \varepsilon}\right) \leq C \text {. }
$$

In $V_{2, \varepsilon}, u_{\varepsilon}$ agrees with $\tilde{u}$, and since $\tilde{u}$ takes values in $S^{k-1}$, the energy density $e_{\varepsilon}\left(u_{\varepsilon}\right)$ reduces to $|D \tilde{u}|^{k} / k$. For every $n$-dimensional face $F$ of $\partial N$ and every $x \in U(F, d, \gamma)$, assumption (iii) implies

$$
|D \tilde{u}(x)|=\left|D \frac{x^{\prime \prime}}{\left|x^{\prime \prime}\right|}\right|=\left|\frac{I}{\left|x^{\prime \prime}\right|}-\frac{x^{\prime \prime} \otimes x^{\prime \prime}}{\left|x^{\prime \prime}\right|^{3}}\right|=\frac{(k-1)^{1 / 2}}{\left|x^{\prime \prime}\right|} .
$$

Since the projection of $V_{2, \varepsilon} \cap U(F, d, \gamma)$ on the affine plane spanned by $F$ is contained in $F \cap \Omega_{d}$, where $\Omega_{d}$ is the closed $d$-neighbourhood of $\Omega$, from (4.6) we obtain

$$
\begin{aligned}
F_{\varepsilon}\left(u_{\varepsilon}, V_{2, \varepsilon} \cap U(F, d, \gamma)\right) & \leq \mathcal{H}^{n}\left(F \cap \Omega_{d}\right) \frac{(k-1)^{k / 2}}{k} \int_{B_{d}^{k} \backslash B_{\varepsilon}^{k}} \frac{d x^{\prime \prime}}{\left|x^{\prime \prime}\right|^{k}} \\
& \leq \mathcal{H}^{n}\left(F \cap \Omega_{d}\right) \frac{(k-1)^{k / 2}}{k} \int_{\varepsilon}^{1} \frac{k \alpha_{k} \rho^{k-1} d \rho}{\rho^{k}} \\
& =\beta_{k}|\log \varepsilon| \cdot \mathcal{H}^{n}\left(F \cap \Omega_{d}\right) .
\end{aligned}
$$

Since the sets $V_{2, \varepsilon} \cap U(F, d, \gamma)$ cover $V_{2, \varepsilon}$ (and have negligible intersections), summing the previous estimate over all $F$ yields

$$
F_{\varepsilon}\left(u_{\varepsilon}, V_{2, \varepsilon}\right) \leq \beta_{k}|\log \varepsilon| \cdot \mathcal{H}^{n}\left(M \cap \Omega_{d}\right) .
$$

It remains to estimate the energy in $V_{3}:=\Omega \backslash U$. Using (4.1) it is easy to check that $\operatorname{dist}(x, S) \leq C \operatorname{dist}(x, M \cup S)$ in the complement of $U$. Thus assumption (ii) becomes $|D \tilde{u}| \leq C /(\operatorname{dist}(x, S))^{p}$, and by formula (4.3)

$$
\left|D u_{\varepsilon}(x)\right| \leq \frac{C}{\operatorname{dist}(x, S)^{p}} .
$$

Taking into account that $W\left(u_{\varepsilon}\right)=0$ in the complement of the $\varepsilon$-neighbourhood of $M \cup S$, and using Lemma 8.3 we obtain

$$
F_{\varepsilon}\left(u_{\varepsilon}, V_{3, \varepsilon}\right) \leq \int_{\Omega} \frac{C d x}{\operatorname{dist}(x, S)^{k p}}+\frac{C}{\varepsilon^{2}} \mathcal{L}^{n+k}\left((M \cup S)_{\varepsilon}\right) \leq C .
$$


Putting together (4.5), (4.7), and (4.8) we obtain

$$
\limsup _{\varepsilon \rightarrow 0} \frac{F_{\varepsilon}\left(u_{\varepsilon}\right)}{|\log \varepsilon|} \leq \beta_{k} \mathcal{H}^{n}\left(M \cap \Omega_{d}\right)=\beta_{k}|M|\left(\Omega_{d}\right) .
$$

Finally, the assumption $|M|(\partial \Omega)=0$ implies that the infimum of $|M|\left(\Omega_{d}\right)$ over all $d \in(0,1 \wedge \delta]$ is $|M|(\bar{\Omega})=|M|(\Omega)=\|M\|_{\Omega}$, and (4.4) is proved.

Remark 4.3. Let $\Sigma$ be a Lipschitz $m$-surface contained in $\bar{\Omega}$ and transversal to $M$ and $S .{ }^{16}$ Then the proof of Lemma 4.2 gives

$$
\limsup _{\varepsilon \rightarrow 0} \frac{1}{|\log \varepsilon|} \int_{\Sigma} e_{\varepsilon}\left(u_{\varepsilon}\right) d \mathcal{H}^{m} \leq C \mathcal{H}^{m-k}(M \cap \Sigma),
$$

where $C$ is a constant that depends only on the choice of $M$ and $\Sigma$.

Proof of Theorem 1.1(ii). Let $M$ be taken as in Lemma 4.2: by Theorem 5.10 in [3] (see also Theorem 9.6 in Appendix B), there exists a map $\tilde{u}$ which satisfies assumptions (i)-(iii) of Lemma 4.2 with $S$ the union of all $(n-1)$-dimensional faces of $N$; in fact, $\tilde{u}$ is defined on $\mathbb{R}^{n+k}$, and satisfies

$$
|D \tilde{u}(x)|=O\left(\frac{1}{\operatorname{dist}(x, \partial N \cup S)}\right) .
$$

Then the maps $u_{\varepsilon}$ defined in (4.3) satisfy statement (ii) of Theorem 1.1-note that (4.4) and (1.3) imply (1.4). The proof for general $M$ follows by the polyhedral approximation given in Proposition $\mathbf{8 . 6}$ and a suitable diagonal argument.

\section{Boundary Value Problems}

In the rest of this paper we deal with Dirichlet boundary conditions. In this section we describe some related notions and then state the $\Gamma$-convergence and compactness result in this setting (Theorem 5.5) and the concentration result for minimizing sequences (Corollary 5.6). Proofs will be given in Section 6 and Section 7.

Here and in the rest of this paper we assume that $n \geq 1$ and $\Omega$ is a bounded Lipschitz domain. Unless otherwise stated, we only consider currents on the entire space $\mathbb{R}^{n+k}$, and convergence will be intended in the sense of the flat norm $\mathbf{F}_{\mathbb{R}^{n+k}}$. In particular, given $u \in W^{1, k}\left(\Omega, \mathbb{R}^{k}\right), \star J u$ denotes now the current in $\mathbb{R}^{n+k}$ which is obtained by extending the Jacobian of $u$ to 0 outside $\Omega$.

\footnotetext{
${ }^{16}$ If $N_{1}$ and $N_{2}$ are finite union of Lipschitz surfaces in $\mathbb{R}^{d}$ of dimension $d_{1}$ and $d_{2}$, respectively, we say that they are transversal when either $d_{1}+d_{2}<d$ and $N_{1} \cap N_{2}$ is empty, or $d_{1}+d_{2} \geq d$ and $N_{1} \cap N_{2}$ is contained in a finite union of Lipschitz surfaces with dimension $d_{1}+d_{2}-d$, and there exists a finite $C$ such that $\operatorname{dist}\left(x, N_{1} \cap N_{2}\right) \leq C \operatorname{dist}\left(x, N_{1}\right)$ for all $x \in N_{2}$, and viceversa. In case of polyhedral sets, this notion is equivalent to $\operatorname{dim}\left(N_{1} \cap N_{2}\right) \leq d_{1}+d_{2}-d$.
} 
5.1. Cobordant currents. We say that the rectifiable $n$-currents $M$ and $M^{\prime}$ are cobordant in the compact set $E$, and write $M \sim_{E} M^{\prime}$, if $M-M^{\prime}=\partial N$ where $N$ is an integral $(n+1)$-current supported in $E$.

If $M \sim_{E} M^{\prime}$ and $E$ is the closure of a bounded Lipschitz domain, by the isoperimetric theorem we can find $N$ supported in $E$ such that $M-M^{\prime}=\partial N$ and $\|N\| \leq C\left\|M-M^{\prime}\right\|^{1+1 / n}$, where the constant $C$ depends only on $E$ (apply Theorem 4.4.2(2) in [20] with $A$ equal to $E, U$ a neighbourhood of $E$ that admits a Lipschitz retraction on $E, B$ and $W$ empty). This fact and the closure theorem for integral currents imply the following closure property of the relation $\sim_{E}$ : given sequences of rectifiable $n$-currents $\left(M_{i}\right)$ and $\left(M_{i}^{\prime}\right)$ which are uniformly bounded in mass, if $M_{i} \sim_{E} M_{i}^{\prime}$ for all $i$ and $M_{i} \rightarrow M, M_{i}^{\prime} \rightarrow M^{\prime}$, then $M \sim_{E} M^{\prime}$.

Clearly $M \sim_{E} M^{\prime}$ implies that $\operatorname{supp}\left(M-M^{\prime}\right) \subset E$ and $\partial M=\partial M^{\prime}$, but the converse may not hold. However, if $E$ is the closure of a bounded Lipschitz domain and the $n$-th integral homology group $H_{n}(E, \mathbb{Z})$ is trivial, then a rectifiable current supported in $E$ is the boundary of a rectifiable current supported in $E$ if and only if it has no boundary, and therefore $M \sim_{E} M^{\prime}$ if and only supp $\left(M-M^{\prime}\right) \subset$ $E$ and $\partial M=\partial M^{\prime}$.

5.2. Jacobians of traces of Sobolev maps. If $v: \partial \Omega \rightarrow \mathbb{R}^{k}$ is a smooth map, then $J v$ and $\star J v$ can be defined as in Sections 2.9 and 2.10, and in particular $\star J v$ is an $(n-1)$-current in $\mathbb{R}^{n+k}$ supported on $\partial \Omega$. Moreover, if $u$ is a smooth extension of $v$ to $\Omega$, then $\star J v$ is the boundary of $\star J u$ (cf. Lemma 6.1). This identity motivates the following general definition due to F.-B. Hang and F.-H. Lin [23] (inspired by a similar notion introduced in [13], see also [36]): the $k$-dimensional Jacobian of a map $v$ in the trace space $W^{1-1 / k, k}\left(\partial \Omega, \mathbb{R}^{k}\right)$ is the current in $\mathcal{D}_{n-1}\left(\mathbb{R}^{n+k}\right)$ defined by

$$
\star J v:=\partial(\star J u),
$$

where $u$ is any map in $W^{1, k}\left(\Omega, \mathbb{R}^{k}\right)$ with trace $v$ on $\partial \Omega$, and $\star J u$ is the current on $\mathbb{R}^{n+k}$ obtained by extending the Jacobian to 0 outside $\Omega$, as already agreed.

As shown by identity (5.2) below, $\star J v$ does not depend on the choice of the extension $u$, and since $\star J u$ has no boundary in $\Omega, \star J v$ is always supported on $\partial \Omega$, and clearly $\mathbf{F}_{\mathbb{R}^{n+k}}(\star J v) \leq\|\star J u\| \leq\|D u\|_{k}^{k}$ (but $\star J v$ may have infinite mass). Moreover, $\star J$ is a continuous operator from $W^{1-1 / k, k}\left(\partial \Omega, \mathbb{R}^{k}\right)$ into the space of $(n-1)$-dimensional boundaries in $\mathbb{R}^{n+k}$ with finite $\mathbf{F}_{\mathbb{R}^{n+k}}$ norm.

Proposition 5.3. Let $\Omega$ be a bounded Lipschitz domain and $v$ a map in $W^{1-1 / k, k}\left(\partial \Omega, \mathbb{R}^{k}\right)$. Take maps $u, u^{\prime} \in W^{1, k}\left(\Omega, \mathbb{R}^{k}\right)$ which extend $v$, and regular level sets $M_{y}(u), M_{y^{\prime}}(u), M_{y}\left(u^{\prime}\right)$ in the sense of Section 2.7. Then

$$
M_{y}(u) \sim \bar{\Omega} M_{y}\left(u^{\prime}\right) .
$$

If in addition $v$ takes values in $S^{k-1}$, then 
(i) $M_{y}(u)$ is cobordant to 0 in $\bar{\Omega}$ when $|y|>1$;

(ii) $M_{y}(u)$ and $M_{y^{\prime}}(u)$ are cobordant in $\bar{\Omega}$ when $|y|,\left|y^{\prime}\right|<1$;

(iii) $M_{y}(u)$ is cobordant in $\bar{\Omega}$ to $(-1)^{n} M_{y^{\prime}}(v)$ when $|y|<1, v$ is of class $W^{1, k-1}$, and $y^{\prime}$ is a regular value of $v$ in the sense of Remark 2.8(ii);

(iv) $\partial M_{y}(u)=\alpha_{k}^{-1} \star J v$ when $|y|<1$; then $\alpha_{k}^{-1} \star J v$ is the boundary of a rectifable current, and is itself rectifiable when it has finite mass.

Remark 5.4. (i) The proof of statement (iii) above (see Section 6) gives a slightly more explicit result: for a.e. $y$ and $y^{\prime}, M_{y}(u)-(-1)^{n} M_{y^{\prime}}(v)$ is the boundary of $M_{e}\left(u_{y}\right)$, where

$$
u_{y}:=\frac{u-y}{|u-y|} \quad \text { and } \quad e:=\frac{y^{\prime}-y}{\left|y^{\prime}-y\right|}
$$

(by Proposition 6.4(ii), the map $u_{y}$ belongs to $W^{1, k-1}\left(\Omega, S^{k-1}\right)$ for a.e. $y$ ). In particular $M_{y}(u)$ agrees in $\Omega$ with $\partial M_{e}\left(u_{y}\right)$.

(ii) The rectifiability of $\alpha_{k}^{-1} \star J v$ (see statement (iv)) was first proved in [23]. We give here a simplified proof.

(iii) If $v$ is a map of class $W^{1, k}$, then $J v$ agrees with the usual Jacobian, namely the pull-back of the standard volume form on $\mathbb{R}^{k}$. Therefore $J v=0$ when $v$ takes values in $S^{k-1}$ because $\mathcal{L}^{k}\left(S^{k-1}\right)=0$. This is not true in general for maps of class $W^{1-1 / k, k}$, and the typical example is $v(x):=x^{\prime} /\left|x^{\prime}\right|$, with $x=\left(x^{\prime}, x^{\prime \prime}\right)$ in $\mathbb{R}^{k} \times \mathbb{R}^{n-1}$, whose Jacobian $\star J v$ is the current associated to the plane $\{0\} \times \mathbb{R}^{n-1}$ and constant multiplicity $\alpha_{k}$.

(iv) If $v \in W^{1-1 / k, k}\left(\partial \Omega, S^{k-1}\right)$ is smooth in the complement of an $(n-1)$ dimensional surface $M \subset \partial \Omega$ without boundary, then $\star J v$ is supported on $M$, and more precisely is of the form $\alpha_{k} \sigma \tau_{M} \cdot \mathcal{H}^{n-1} \mathrm{~L} M$, where $\sigma$ is an integral multiplicity that can be explicitly computed in terms of the degree of the restriction of $u$ to certain $k$-surfaces (cf. [28] and [3, Section 3.7]).

We can now state the main results of this section.

Theorem 5.5. Let $\Omega$ be a bounded Lipschitz domain and let $v$ be a map in $W^{1-1 / k, k}\left(\partial \Omega, S^{k-1}\right)$. Choose $u \in W^{1, k}\left(\Omega, \mathbb{R}^{k}\right)$ with trace $v$ on $\partial \Omega$ and a regular level set $M_{y}=M_{y}(u)$ with $|y|<1$. The following statements hold.

(i) Assume a (countable) sequence of maps $u_{\varepsilon} \in W^{1, k}\left(\Omega \cdot \mathbb{R}^{k}\right)$ with trace $v$ on $\partial \Omega$ such that $F_{\varepsilon}\left(u_{\varepsilon}\right)=O(|\log \varepsilon|)$. Then we can extract a subsequence such that the Jacobians $\star J u_{\varepsilon}$ converge in the flat norm $\mathbf{F}_{\mathbb{R}^{n+k}}$ to $\alpha_{k} M$, where $M$ is a rectifiable $n$-current supported in $\bar{\Omega}$ such that $M \sim \bar{\Omega} M_{y}$. The following localized version of the lower bound inequality holds: for every open set $A \subset \mathbb{R}^{n+k}$

$$
\liminf _{\varepsilon \rightarrow 0} \frac{F_{\varepsilon}\left(u_{\varepsilon}, A \cap \Omega\right)}{|\log \varepsilon|} \geq \beta_{k}\|M\|_{A} .
$$


(ii) Given a rectifiable $n$-current $M$ supported in $\bar{\Omega}$ such that $M \sim \bar{\Omega} M_{y}$, for every $\varepsilon>0$ we can find $u_{\varepsilon}$ such that $u_{\varepsilon}=v$ on $\partial \Omega, \mathbf{F}_{\mathbb{R}^{n+k}}\left(\star J u_{\varepsilon}-\alpha_{k} M\right) \rightarrow 0$, and

$$
\lim _{\varepsilon \rightarrow 0} \frac{F_{\varepsilon}\left(u_{\varepsilon}, \Omega\right)}{|\log \varepsilon|}=\beta_{k}\|M\|_{\mathbb{R}^{n+k}}
$$

Remarks. (i) By Proposition 5.3, the cobordancy condition $M \sim \bar{\Omega} M_{y}(u)$ in Theorem 5.5 (and in Corollary 5.6 below) is independent of the choice of the extension $u$ and of the value $y$. If $v$ is of class $W^{1, k-1}$, by Proposition 5.3(iii) we can replace $M_{y}(u)$ by any regular level set of $v$ in the sense of Remark 2.8(ii).

(ii) Statement (i) of Theorem 5.5 is stronger than the corresponding statement in Theorem 1.1 under many regards. First of all, the rectifiable current $M$ is the limit of the Jacobians of $u_{\varepsilon}$ in $\mathbb{R}^{n+k}$, and not just in $\Omega$ : it may happen that part of $M$ is supported on the boundary of $\partial \Omega$, and in that case the rectifiable current given by statement (ii) of Theorem 1.1 is the restriction of $M$ to $\Omega$. Accordingly, the right-hand side of (5.3) takes also into account the part of the mass of $M$ which is located on the boundary of $\Omega$ and therefore (5.3) cannot be deduced from (1.3) except when $A \cap \partial \Omega=\varnothing$.

(iii) As already pointed out in Remark (i) after Theorem 1.1, the right-hand side of (5.3) does not depend on the choice of the potential $W$. Hence, replacing $W$ with $\sigma W$ and then taking the limit in (5.3) as $\sigma \rightarrow 0$, we obtain the stronger estimate

$$
\liminf _{\varepsilon \rightarrow 0} \frac{1}{|\log \varepsilon|} \int_{\Omega \cap A} \frac{1}{k}\left|D u_{\varepsilon}\right|^{k} \geq \beta_{k}\|M\|_{A} .
$$

In particular, if the sequence $\left(u_{\varepsilon}\right)$ satisfies the upper bound inequality (5.4), then the potential part of the energy is asymptotically negligible, in the sense that (1.5) holds.

Corollary 5.6. Take $\Omega, v, u, M_{y}$ as in Theorem 5.5, and denote by $m_{\varepsilon}$ the minimum of $F_{\varepsilon}(u, \Omega)$ over all maps $u \in W^{1, n}\left(\Omega, \mathbb{R}^{k}\right)$ with trace $v$ on $\partial \Omega$. Then

$$
m_{\varepsilon}+O(|\log \varepsilon|) .
$$

Let $\left(u_{\varepsilon}\right)$ be any (countable) sequence of maps with trace $v$ on $\partial \Omega$ such that

$$
F_{\varepsilon}\left(u_{\varepsilon}, \Omega\right)=m_{\varepsilon}+o(|\log \varepsilon|) .
$$

Then, up to a subsequence, the Jacobians $\star J u_{\varepsilon}$ converge in the flat norm $\mathbf{F}_{\mathbb{R}^{n+k}}$ to $\alpha_{k} M$, where $M$ minimizes the mass among all rectifiable $n$-current supported in $\bar{\Omega}$ which are cobordant to $M_{y}$ in $\bar{\Omega}$. Moreover, the potential part of the energy is asymptotically negligible, in the sense that (1.5) holds, and the energy densities $e_{\varepsilon}\left(u_{\varepsilon}\right)$ and $\left|D u_{\varepsilon}\right|^{k} / k$, rescaled by $1 /|\log \varepsilon|$, converge to $\beta_{k}|M|$ in the sense of measures on $\mathbb{R}^{n+k}$. 

implies

Remarks. (i) By Proposition 5.3(iv), the cobordancy condition $M \sim \bar{\Omega} M_{y}$

$$
\partial M=\alpha_{k}^{-1} \star J v .
$$

When the $n$-th homology group $H_{n}(\bar{\Omega}, \mathbb{Z})$ is trivial, $M \sim \bar{\Omega} M_{y}$ is equivalent to $\partial M=\alpha_{k}^{-1} \star J v$ (see Section 5.1). Thus Corollary 1.2 is a particular case of Corollary 5.6.

(ii) If $\star J v$ vanishes—which happens, e.g., when $v$ is of class $W^{1, k}$-then the current $M$ in Corollary 5.6 minimizes the area among all cycles (rectifiable currents without boundary) in the integral homology class of $M_{y}$. If in addition $H_{n}(\bar{\Omega}, \mathbb{Z})=0$, then $M$ is trivial.

\section{Proof of Proposition 5.3 AND Theorem 5.5(i)}

We prove first Proposition 5.3: even though all claims are obvious (or empty) when $u$ and $u^{\prime}$ are smooth up to the boundary, the proof in the general case is rather delicate. We follow the notation of Section 5 .

Lemma 6.1. Let $u$ be a map in $W^{1, k}\left(\Omega, \mathbb{R}^{k}\right)$ with trace $v$ in $W^{1, k}\left(\partial \Omega, \mathbb{R}^{k}\right)$, and let $\rho$ be a bounded continuous function on $\mathbb{R}^{k}$. Then

$$
\partial(\rho(u) \star J u)=\rho(v) \star J v \quad \text { in } \mathbb{R}^{n+k} .
$$

Proof. By a density argument, it suffices to prove (6.1) when $u$ is smooth up to the boundary. Denoting by $\tau_{\Omega}=e_{1} \wedge \cdots \wedge e_{n+k}$ the standard orientation of $\Omega$, for every $(n-1)$-form $\omega \in \mathcal{D}^{n-1}\left(\mathbb{R}^{n+k}\right)$ there holds

$$
\begin{aligned}
(\rho(u) \star J u)[d \omega] & =\int_{\Omega}(d \omega \wedge \rho(u) J u) \cdot \tau_{\Omega}=\int_{\Omega} d(\omega \wedge \rho(u) J u) \cdot \tau_{\Omega} \\
& =\int_{\partial \Omega}(\omega \wedge \rho(u) J v) \cdot \tau_{\partial \Omega}=(\rho(u) \star J v)[\omega],
\end{aligned}
$$

where the first and fourth identities follow from (2.9), the third one is Stokes theorem, and the second one is a particular case of the general identity $d(\omega \wedge$ $\left.\omega^{\prime}\right)=d \omega \wedge \omega^{\prime}+(-1)^{m} \omega \wedge d \omega^{\prime}$, where $m$ is the order of the form $\omega$ (in this case $m:=n-1$ and $\omega^{\prime}:=\rho(u) J u=u^{\sharp}(\rho d y)$, thus $d \omega^{\prime}=u^{\sharp}(d(\rho d y))=$ $\left.u^{\#}(0)=0\right)$.

Lemma 6.2. Assume a bounded continuous function $\rho$ on $\mathbb{R}^{k}$, and maps $u, u^{\prime}$ in $W^{1, k}\left(\Omega, \mathbb{R}^{k}\right)$. We denote by $\pi$ the projection of $\mathbb{R} \times \mathbb{R}^{n+k}$ on $\mathbb{R}^{n+k}$, and set

$$
\begin{aligned}
U(t, x) & :=t u(x)+(1-t) u^{\prime}(x) & & \text { for every }(t, x) \in(0,1) \times \Omega, \\
\bar{\rho}(y) & :=\int_{0}^{1} \rho(t y) t^{k-1} d t & & \text { for every } y \in \mathbb{R}^{k} .
\end{aligned}
$$


Thus $\pi_{\sharp} \partial(\rho(U) \star J U)$ is an $n$-current on $\mathbb{R}^{n+k}$ of class $L^{1}$. If $u$ and $u^{\prime}$ have the same trace on $\partial \Omega$, then

$$
\pi_{\sharp} \partial(\rho(U) \star J U)=\rho(u) \star J u-\rho\left(u^{\prime}\right) \star J u^{\prime} \quad \text { in } \mathbb{R}^{n+k},
$$

whereas if $u$ has trace $v \in W^{1, k-1}\left(\partial \Omega, \mathbb{R}^{k}\right)$ and $u^{\prime}=0$, then ${ }^{17}$

$$
\pi_{\sharp} \partial(\rho(U) \star J U)=\rho(u) \star J u-(-1)^{n} \star v^{\sharp}\left(\bar{\rho} \omega_{0}\right) \text { in } \mathbb{R}^{n+k} .
$$

Proof. By a density argument, we can assume that the traces of $u$ and $u^{\prime}$ belong to $W^{1, k}\left(\partial \Omega, \mathbb{R}^{k}\right)$. If we denote by $V$ the trace of $U$ on the boundary of $A:=(0,1) \times \Omega$, Lemma 6.1 yields

$$
\pi_{\#} \partial(\rho(U) \star J U)=\pi_{\#}(\rho(V) \star J V) .
$$

The boundary of $A$ is the union of $\{1\} \times \Omega,\{0\} \times \Omega$, and $(0,1) \times \partial \Omega$, and we denote the restrictions of $\rho(V) \star J V$ to these three sets by $T_{1}, T_{2}$ and $T_{3}$. We claim that

$$
\pi_{\sharp} T_{1}=\rho(u) \star J u \quad \text { and } \quad \pi_{\sharp} T_{2}=-\rho\left(u^{\prime}\right) \star J u^{\prime} .
$$

Indeed, since $V=u$ and $\tau_{\partial A}=\tau_{\Omega}$ on $\{1\} \times \Omega$ (cf. Note (11)), for every smooth $n$-form $\omega$ on $\Omega$ there holds

$$
\begin{aligned}
\pi_{\sharp} T_{1}[\omega]=T_{1}\left[\pi^{\sharp} \omega\right] & =\int_{\{1\} \times \Omega}\left(\pi^{\sharp} \omega \wedge \rho(V) J V\right) \cdot \tau_{\partial A} \\
& =\int_{\Omega}(\omega \wedge \rho(u) J u) \cdot \tau_{\Omega}=(\rho(u) \star J u)[\omega] .
\end{aligned}
$$

A similar argument gives the second identity in (6.5).

We compute now $T_{3}$. Let $e$ be the unit vector that orients $\mathbb{R} \times\{0\}$. Then $\tau_{\partial A}=-e \wedge \tau_{\partial \Omega}$ on $(0,1) \times \partial \Omega$. If we assume that $u$ and $u^{\prime}$ have the same trace $v$ on $\partial \Omega$, then $V(t, x)=v(x)$ and $J V(t, x)=J v(x)$ on $(0,1) \times \partial \Omega$, and therefore $^{18}$

$$
\pi_{\sharp} T_{3}[\omega]=\int_{(0,1) \times \partial \Omega} \rho(v)(\omega \wedge J v) \cdot\left(-e \wedge \tau_{\partial \Omega}\right)=0 .
$$

Recalling (6.5), (6.4) and identity $\rho(V) \star J V=T_{1}+T_{2}+T_{3}$ we obtain (6.2).

On the other hand, if we assume $u^{\prime}=0$, then $V(t, x)=t v(x)$ on $(0,1) \times \partial \Omega$ and an easy computation yields

$$
J V=\bigwedge_{i}\left(t d v_{i}+v_{i} d t\right)=t^{k} J v+t^{k-1} d t \wedge v^{\sharp} \omega_{0} .
$$

\footnotetext{
${ }^{17}$ Here $\omega_{0}$ is the $(k-1)$-form defined in (2.7). Thus $v^{\#}(\bar{\rho} \omega)$ is a $(k-1)$-form on $\partial \Omega$ and $\star v^{\sharp}(\bar{\rho} \omega)$ is an $n$-current supported on $\partial \Omega$.

${ }^{18}$ Since no component of $\omega \wedge J v$ contains $d t,(\omega \wedge J v) \cdot(e \wedge \tau)=0$ for every $(n-1)$-vector $\tau$.
} 
Proceeding as before,

$$
\begin{aligned}
\pi_{\sharp} T_{3}[\omega] & =\int_{(0,1) \times \partial \Omega} \rho(t v)\left(t^{k} \omega \wedge J v+t^{k-1} \omega \wedge d t \wedge v^{\sharp} \omega_{0}\right) \cdot\left(-e \wedge \tau_{\partial \Omega}\right) \\
& =\int_{(0,1) \times \partial \Omega}(-1)^{n+1} \rho(t v) t^{k-1}\left(d t \wedge \omega \wedge v^{\sharp} \omega_{0}\right) \cdot\left(e \wedge \tau_{\partial \Omega}\right) \\
& =(-1)^{n+1} \int_{\partial \Omega} \bar{\rho}(v)\left(\omega \wedge v^{\sharp} \omega_{0}\right) \cdot \tau_{\partial \Omega}=(-1)^{n+1} \star v^{\sharp}\left(\bar{\rho} \omega_{0}\right)[\omega],
\end{aligned}
$$

and (6.3) is proved.

Proof of Proposition 5.3. We first prove (5.2). Let $y$ be a regular value of $u$, $u^{\prime}$ and of the map $U$ defined in Lemma 6.2. Take a smooth non-negative function $\rho: \mathbb{R}^{k} \rightarrow \mathbb{R}$ with compact support and integral 1 , and set $\rho_{\varepsilon}(z):=\varepsilon^{-k} \rho((z-$ $y) / \varepsilon$ ). Using the coarea formula (2.6), and replacing $\rho$ with $\rho_{\varepsilon}$, identity (6.2) can be rewritten as

$$
\int_{\mathbb{R}^{k}} \rho_{\varepsilon}(z) \partial\left(\pi_{\#} M_{z}(U)\right) d z=\int_{\mathbb{R}^{k}} \rho_{\varepsilon}(z)\left(M_{z}(u)-M_{\mathcal{z}}\left(u^{\prime}\right)\right) d z,
$$

and passing to the limit as $\varepsilon \rightarrow 0$ we obtain (5.2). A density argument and the closure property of the cobordism relation give (5.2) when $y$ is not a regular value of $U$.

Statement (i) follows from (5.2) by taking $u^{\prime}:=\Phi(u)$, where $\Phi$ agrees with the identity on $S^{k-1}$ (so that $u=u^{\prime}$ on $\partial \Omega$ ), and projects $\mathbb{R}^{k}$ onto the closed unit ball (so that $M_{y}\left(u^{\prime}\right)=0$ ).

Statement (ii) follows from (5.2) by taking $u^{\prime}:=\Phi(u)$, where $\Phi$ is a smooth diffeomorphism that agrees with the identity on $S^{k-1}$ and takes $y^{\prime}$ into $y$ (so that $\left.M_{y}\left(u^{\prime}\right)=M_{y^{\prime}}(u)\right)$.

The coarea formula (2.6) yields

$$
\star J v=\partial(\star J u)=\int_{\mathbb{R}^{k}} \partial M_{y}(u) d y,
$$

and since the currents $M_{y}(u)$ have all the same boundary for $|y|<1$, and no boundary for $|y|>1$, we obtain that $\star J v=\alpha_{k} \partial M_{y}(u)$. The rest of statement (iv) follows by the boundary rectifiability theorem.

It remains to prove statement (iii). Let $y$ be a regular value of $u$ and $U$, and assume that $y^{\prime}:=y /|y|$ is a regular value of $v$. Using the coarea formula (2.6) and the coarea formula for maps in $W^{1, k-1}\left(\partial \Omega, S^{k-1}\right)$ (see [3, Section 7.5]), and replacing $\rho$ with the function $\rho_{\varepsilon}$ defined above, identity (6.3) can be rewritten as

$$
\begin{aligned}
& \int_{\mathbb{R}^{k}} \rho_{\varepsilon}(z) \partial\left(\pi_{\sharp} M_{\mathcal{z}}(U)\right) d z \\
& \quad=\int_{\mathbb{R}^{k}} \rho_{\varepsilon}(z) M_{\mathcal{Z}}(u) d z-(-1)^{n} \int_{S^{k-1}} \bar{\rho}_{\varepsilon}\left(z^{\prime}\right) M_{\mathcal{z}^{\prime}}(u) d \mathcal{H}^{k-1}\left(z^{\prime}\right),
\end{aligned}
$$


and passing to the limit as $\varepsilon \rightarrow 0$ we obtain $\partial\left(\pi_{\sharp} M_{y}(U)\right)=M_{y}(u)-M_{y^{\prime}}(v)$, which implies $M_{y}(u) \sim \bar{\Omega} M_{y^{\prime}}(v)$. We prove statement (iii) in full generality using statement (ii) and a density argument.

6.3. Construction of maps with good energy bounds. For every $a \in \mathbb{R}^{k}$ and $\varepsilon \geq 0$ such that $\varepsilon+|a|<1$ we choose a map $\Phi_{a, \varepsilon}: \mathbb{R}^{k} \rightarrow \mathbb{R}^{k}$ that maps the open ball $B_{a, \varepsilon}:=(1-\varepsilon) a+B_{\varepsilon}^{k}$ onto the unit open ball $B_{1}^{k}$ homothetically, maps the rest of $\mathbb{R}^{k}$ in the sphere $S^{k-1}$, and agrees with the identity on $S^{k-1}$.

Here is a more precise definition: we set $\Phi_{a, \varepsilon}(a):=a$, then we write every $y \in \mathbb{R}^{k}$ different from $a$ as $a+t v$ with $t>0$ and $v$ such that $a+v \in S^{k-1}$, and set

$$
\Phi_{a, \varepsilon}(y):= \begin{cases}a+\frac{1}{\varepsilon} t v & \text { for } t<\varepsilon, \\ a+v & \text { for } t \geq \varepsilon .\end{cases}
$$

In particular, $\Phi_{a, \varepsilon}(y)=a+(y-a) / \varepsilon$ for every $y \in B_{a, \varepsilon}$. Moreover, $\Phi_{a, \varepsilon}$ is Lipschitz for $\varepsilon>0$, and locally Lipschitz on $\mathbb{R}^{k} \backslash\{a\}$ for $\varepsilon=0$. A lengthy but straightforward computation yields

$$
\left|D \Phi_{a, \varepsilon}(y)\right| \leq \frac{K}{|y-a| \vee \varepsilon} \quad \text { for a.e. } y .
$$

For every $u: \Omega \rightarrow \mathbb{R}^{k}$ we set

$$
u_{a}=u_{a, 0}:=\Phi_{a, 0}(u) \quad \text { and } \quad u_{a, \varepsilon}:=\Phi_{a, \varepsilon}(u) .
$$

The following lemma was inspired by the proof of Proposition 4 in [8], based in turn on computations from [24].

Proposition 6.4. Take $u, a, \varepsilon, u_{a}, u_{a, \varepsilon}, B_{a, \varepsilon}$ as above.

(i) The map $u_{a, \varepsilon}$ belongs to $W^{1, k}\left(\Omega, \mathbb{R}^{k}\right)$ for all $a$ and all $\varepsilon>0$, and

$$
\star J u_{a, \varepsilon}=\frac{1}{\varepsilon^{k}} \int_{B_{a, \varepsilon}} M_{y}(u) d y .
$$

(ii) The map $u_{a}$ belongs to $W^{1, k-1}\left(\Omega, S^{k-1}\right)$ for a.e. $a \in B_{1}^{k}$, and $\star J u_{a}=$ $\alpha_{k} M_{a}(u)$.

(iii) There exists a universal constant $K$ such that, for every $\varepsilon>0$,

$$
\begin{aligned}
& \int_{B_{1-\varepsilon}^{k}} F_{\varepsilon}\left(u_{a, \varepsilon}\right) d a \leq K\left[|\log \varepsilon| \cdot\|D u\|_{k}^{k}+\varepsilon^{k-2} \mathcal{L}^{n+k}(\Omega)\right], \\
& \int_{B_{1-\varepsilon}^{k}}\left\|D u_{a, \varepsilon}-D u_{a}\right\|_{k-1}^{k-1} d a \leq K \varepsilon\|D u\|_{k-1}^{k-1} .
\end{aligned}
$$


(iv) for every $\delta>0$ there exists $E_{\delta} \subset B_{1}^{k}$ with $\mathcal{L}^{k}\left(E_{\delta}\right) \leq \delta$ such that

$$
\liminf _{\varepsilon \rightarrow 0} \frac{F_{\varepsilon}\left(u_{a, \varepsilon}\right)}{|\log \varepsilon|} \leq \frac{K}{\delta}\|D u\|_{k}^{k} \quad \text { for every } a \in B_{1}^{k} \backslash E_{\delta} .
$$

(v) For every $a \in B_{1}^{k}, u_{a, \varepsilon} \rightarrow u_{a}$ pointwise as $\varepsilon \rightarrow 0$, and for a.e. $a \in B_{1}^{k}$

$$
\begin{aligned}
& \liminf _{\varepsilon \rightarrow 0}\left\|u_{a, \varepsilon}-u_{a}\right\|_{W^{1, k-1}}=0, \\
& \liminf _{\varepsilon \rightarrow 0} \mathbf{F}_{\Omega}\left(\star J u_{a, \varepsilon}-\star J u_{a}\right)=0 .
\end{aligned}
$$

(vi) If the trace of $u$ on $\partial \Omega$ takes values in $S^{k-1}$, for a.e. $a \in B_{1}^{k}$ there holds

$$
\liminf _{\varepsilon \rightarrow 0} \mathbf{F}_{\mathbb{R}^{n+k}}\left(\star J u_{a, \varepsilon}-\alpha_{k} M_{a}(u)\right)=0 .
$$

Remarks. (i) As shown in [3, Theorem 3.8], if $u_{a}$ is of class $W^{1, k-1}$, then $\star J u_{a}=(-1)^{n+1} \partial M_{\bar{y}}\left(u_{a}\right)$ for every $\bar{y} \in S^{k-1}$ which is a regular value of $u_{a}$ in the sense of Remark 2.8(ii).

(ii) Statements (iv)-(vi) hold even when applied to a subsequence of $(\varepsilon)$. However, the set of all $a$ for which one among (6.11)-(6.14) fails may depend on the choice of the subsequence, and therefore we cannot infer that the liminf in (6.11)-(6.14) can be replaced by a limsup. As far as we know, these stronger versions of statements (iv)-(vi) could be true. If so, some of the proofs below could be simplified.

Proof. The first part of statement (i) follows by the fact that $\Phi_{a, \varepsilon}$ is Lipschitz for $\varepsilon>0$, while (6.8) follows by the coarea formula (2.6) and the fact that $\operatorname{det}\left(D \Phi_{a, \varepsilon}\right)$ is equal to $\varepsilon^{-k}$ on the ball $B_{a, \varepsilon}$, and vanishes elsewhere.

In order to prove (6.9), we define the following auxiliary function:

$$
\sigma_{\varepsilon}(a, x):= \begin{cases}1 & \text { if }|u(x)-a| \leq 2 \varepsilon, \\ 0 & \text { otherwise. }\end{cases}
$$

Thus (6.6) implies $\left|D u_{a, \varepsilon}\right| \leq K \varepsilon^{-1}|D u|$ for a.e. $x$ such that $\sigma_{\varepsilon}(a, x)=1$, while $\left|D u_{a, \varepsilon}\right| \leq K|u-a|^{-1}|D u|$ for a.e. $x$ such that $\sigma_{\varepsilon}(a, x)=0$. Moreover, in the latter case we also have $u_{a, \varepsilon} \in S^{k-1}$, because $\Phi_{a, \varepsilon}$ maps the complement of $B_{a, \varepsilon}$ into $S^{k-1}$. Hence

$$
\begin{aligned}
F_{\varepsilon}\left(u_{a, \varepsilon}\right) \leq K \int_{\Omega} \sigma_{\varepsilon}(a, x)\left(\frac{|D u(x)|^{k}}{\varepsilon^{k}}\right. & \left.+\frac{1}{\varepsilon^{2}}\right) \\
& +\left(1-\sigma_{\varepsilon}(a, x)\right) \frac{|D u(x)|^{k}}{|u(x)-a|^{k}} d x
\end{aligned}
$$


Now we integrate both sides of (6.15) over all $a$ such that $|a|+\varepsilon<1$, and change the order of integration in the right-hand side:

$$
\begin{aligned}
\int_{B_{1-\varepsilon}^{k}} F_{\varepsilon}\left(u_{a, \varepsilon}\right) d a \leq K \int_{\Omega}\left[\left(\frac{|D u(x)|^{k}}{\varepsilon^{k}}\right.\right. & \left.+\frac{1}{\varepsilon^{2}}\right) \int_{B_{1-\varepsilon}^{k}} \sigma_{\varepsilon}(a, x) d a \\
& \left.+|D u(x)|^{k} \int_{B_{1-\varepsilon}^{k}} \frac{1-\sigma_{\varepsilon}(a, x)}{|u(x)-a|^{k}} d a\right] d x .
\end{aligned}
$$

Thus (6.9) follows by the estimates

$$
\int_{B_{1-\varepsilon}^{k}} \sigma_{\varepsilon}(x, a) d a \leq \mathcal{L}^{k}\left(B_{2 \varepsilon}^{k}\right)=2^{k} \alpha_{k} \varepsilon^{k}
$$

and

$$
\int_{B_{1-\varepsilon}^{k}} \frac{1-\sigma_{\varepsilon}(x, a)}{|u(x)-a|^{k}} d a \leq \int_{B_{3 / 2}^{k} \backslash B_{\varepsilon}^{k}} \frac{d y}{|y|^{k}} \leq K \int_{\varepsilon}^{3 / 2} \frac{d \rho}{\rho} \leq K|\log \varepsilon| .
$$

To prove (6.10), we use that $\left|D u_{a, \varepsilon}\right| \leq K \varepsilon^{-1}|D u|$ and $\left|D u_{a}\right| \leq K|u-a|^{-1}|D u|$ for a.e. $x$ such that $\sigma_{\varepsilon}(a, x)=1$, while $D u_{a, \varepsilon}=D u_{a}$ for a.e. $x$ such that $\sigma_{\varepsilon}(a, x)=0$ because $u_{a, \varepsilon}=u_{a}$ in the complement of $B_{a, \varepsilon}$. Hence

$\int_{\Omega}\left|D u_{a, \varepsilon}-D u_{a}\right|^{k-1} \leq K \int_{\Omega} \sigma_{\varepsilon}(a, x)\left(\frac{1}{\varepsilon^{k-1}}+\frac{1}{|u(x)-a|^{k-1}}\right)|D u(x)|^{k-1} d x$.

The rest of the proof of (6.10) follows that of (6.9). Estimate (6.9) and Fatou's lemma imply

$$
\int_{B_{1}^{k}} \liminf _{\varepsilon \rightarrow 0} \frac{F_{\varepsilon}\left(u_{a, \varepsilon}\right)}{|\log \varepsilon|} d a \leq \liminf _{\varepsilon \rightarrow 0} \int_{B_{1}^{k}} \frac{F_{\varepsilon}\left(u_{a, \varepsilon}\right)}{|\log \varepsilon|} d a \leq K\|D u\|_{k}^{k},
$$

which yields (6.11). In a similar way, we use (6.10) to obtain (6.12), which implies that, up to a subsequence, the maps $u_{a, \varepsilon}$ converge strongly to $u_{a}$ in $W^{1, k-1}$ (and in particular $u_{a}$ belongs to $W^{1, k-1}$ ). Now (6.13) follows by the fact that strong convergence in $W^{1, k-1}$, implies convergence of Jacobians in the flat norm $\mathbf{F}_{\Omega}$ (see Remark 2.11).

The convergence of $\star J u_{a, \varepsilon}$ to $\star J u_{a}$ (up to a subsequence) and formula (6.8) imply $\star J u_{a}=\alpha_{k} M_{a}(u)$ for every regular value $a$ of $u$, and the proof of (ii) is completed.

It remains to prove (vi). We take a regular value $a$ of $u$ such that $\star J u_{a}=$ $\alpha_{k} M_{a}(u)$ (cf. statement (ii)) and $u_{a, \varepsilon}$ converge to $u_{a}$ in $W^{1, k-1} u p$ to a subsequence, not relabelled in the following (cf. (6.12)). Now we choose a map $\tilde{u}$ in $W^{1, k}\left(\mathbb{R}^{n+k} \backslash \bar{\Omega}, \mathbb{R}^{k}\right)$ with the same trace as $u$ on $\partial \Omega$ and for every $\varepsilon>0$ we set

$$
\tilde{u}_{a, \varepsilon}(x):= \begin{cases}u_{a, \varepsilon}(x) & \text { if } x \in \Omega, \\ \tilde{u}(x) & \text { if } x \in \mathbb{R}^{n+k} \backslash \bar{\Omega},\end{cases}
$$


and define $\tilde{u}_{a}$ in the same way. Since trace of $u_{a, \varepsilon}$ and $u_{a}$ on $\partial \Omega$ agrees with that of $u$, and therefore with that of $\tilde{u}$, the maps $\tilde{u}_{a, \varepsilon}$ belong to $W^{1, k}\left(\mathbb{R}^{n+k}, \mathbb{R}^{k}\right)$ and converge in $W^{1, k-1}\left(\mathbb{R}^{n+k}, \mathbb{R}^{k}\right)$ to $\tilde{u}_{a}$. Hence

$$
\mathbf{F}_{\mathbb{R}^{n+k}}\left(\star J \tilde{u}_{a, \varepsilon}-\star J \tilde{u}_{a}\right) \rightarrow 0 .
$$

On the other hand, $\star J \tilde{u}_{a, \varepsilon}$ can be decomposed as $\star J u \tilde{u}+\star J u_{a, \varepsilon}$ on $\mathbb{R}^{n+k}$ (we assume that $\star J \tilde{u}$ is extended to 0 in $\bar{\Omega}$, while $\star J u_{a, \varepsilon}$ is extended to 0 outside $\Omega$ ). Now, the first addendum agrees with the restriction of $\star J \tilde{u}_{a}$ to $\mathbb{R}^{n+k} \backslash \bar{\Omega}$, while formula (6.8) and the fact that $a$ is a regular value of $u$ imply that the second addendum converges to $\alpha_{k} M_{a}(u)$ in the weak topology of currents. In particular, $\alpha_{k} M_{a}(u)$ must agree with the restriction of $\star J \tilde{u}_{a}$ to $\bar{\Omega}$, and then (6.17) yields (6.14) (recall that (6.17) holds for a subsequence only).

Proof of statement (i) of Theorem 5.5. Let a (countable) sequence $\left(u_{\varepsilon}\right)$ be given such that $u_{\varepsilon}=v$ on $\partial \Omega$ and $F_{\varepsilon}\left(u_{\varepsilon}\right)=O(|\log \varepsilon|)$, and an open set $A \subset \mathbb{R}^{n+k}$. As in the proof of statement (i) of Theorem 1.1, we can assume that the liminf at the left-hand side of (5.3) is a limit.

First step: compactness and lower bound inequality. The idea is to apply statement (i) of Theorem 1.1 with $\Omega$ replaced by a larger set (and $u_{\varepsilon}$ suitably extended outside of $\Omega$ ). Let $\delta>0$ be fixed for the time being. First we take $u \in W^{1, k}\left(\mathbb{R}^{n+k}, \mathbb{R}^{k}\right)$ with trace $v$ on $\partial \Omega$. Then we choose an open set $\Omega^{\prime}$ so that $\Omega \Subset \Omega^{\prime}$ and

$$
\int_{\Omega^{\prime} \backslash \Omega}|D u|^{k} \leq \delta .
$$

We set $U:=\Omega^{\prime} \backslash \bar{\Omega}$. Now, let $u_{a, \varepsilon}$ be defined as in (6.7). By Proposition 6.4 we can choose $a \in B_{1}^{k}$ so that, passing to a subsequence,

$$
\begin{aligned}
& F_{\varepsilon}\left(u_{a, \varepsilon}, \Omega^{\prime}\right)=O(|\log \varepsilon|), \\
& F_{\varepsilon}\left(u_{a, \varepsilon}, U\right) \leq K|\log \varepsilon| \int_{U}|D u|^{k} \leq K \delta|\log \varepsilon|, \\
& \mathbf{F}_{\Omega^{\prime}}\left(\star J u_{a, \varepsilon}-\alpha_{k} M_{a}(u)\right) \rightarrow 0, \\
& \mathbf{F}_{\mathbb{R}^{n+k}}\left(\star J u _ { a , \varepsilon } \left\llcornerU-\alpha_{k} M_{a}(u)\llcorner U) \rightarrow 0 .\right.\right.
\end{aligned}
$$

Finally, we set

$$
u_{\varepsilon}^{\prime}(x):= \begin{cases}u_{\varepsilon}(x) & \text { if } x \in \Omega, \\ u_{a, \varepsilon}(x) & \text { if } x \in \Omega^{\prime} \backslash \Omega .\end{cases}
$$

The maps $u_{\varepsilon}^{\prime}$ belong to $W^{1, k}\left(\Omega^{\prime}, \mathbb{R}^{k}\right)$, and $F\left(u_{\varepsilon}^{\prime}, \Omega^{\prime}\right)=O(|\log \varepsilon|)$ by (6.19). By Theorem 1.1(i) implies that, up to a subsequence, $\star J u_{\varepsilon}^{\prime}$ converge to $\alpha_{k} M^{\prime}$ in the flat norm $\mathbf{F}_{\Omega^{\prime}}$, where $M^{\prime}$ is a rectifiable current supported in $\Omega^{\prime}$. Then (6.21) 
implies that $\star J u_{\varepsilon}=\star J u_{\varepsilon}^{\prime}-\star J u_{a, \varepsilon}\left\llcorner U\right.$ converge in the flat norm $\mathbf{F}_{\Omega^{\prime}}$ to $\alpha_{k} M$, where

$$
M:=M^{\prime}-M_{a}(u)\left\llcorner U=M^{\prime}\llcorner\bar{\Omega} .\right.
$$

Since $\star J u_{\varepsilon}$ and $M$ are supported in $\bar{\Omega}$, we have convergence in the flat norm $\mathbf{F}_{\mathbb{R}^{n+k}} \cdot{ }^{19}$ Moreover, the lower bound inequality (1.3) applied to the maps $u_{\varepsilon}^{\prime}$ and to the set $\Omega^{\prime} \cap A$ and estimate (6.19) yield

$$
\begin{aligned}
\beta_{k}|M|(A) & =\beta_{k}\left|M^{\prime}\right|(A \cap \bar{\Omega}) \leq \beta_{k}\left|M^{\prime}\right|\left(A \cap \Omega^{\prime}\right) \\
& \leq \liminf _{\varepsilon \rightarrow 0} \frac{F_{\varepsilon}\left(u_{\varepsilon}^{\prime}, A \cap \Omega^{\prime}\right)}{|\log \varepsilon|} \leq \liminf _{\varepsilon \rightarrow 0} \frac{F_{\varepsilon}\left(u_{\varepsilon}, A \cap \Omega\right)}{|\log \varepsilon|}+K \delta .
\end{aligned}
$$

Since $\delta$ can be taken arbitrarily small, the lower bound inequality (5.3) is proved.

Second step: $M$ is cobordant to $M_{a}(u)\llcorner\Omega$ in $\bar{\Omega}$. While it is easy to prove that these two currents differ by the boundary of a real current with finite mass supported in $\bar{\Omega}$, it is more difficult to show that they differ by a rectifiable one.

Choose an open set $V$ such that $\Omega \Subset V \Subset \Omega^{\prime}$ and whose closure admits a Lipschitz retraction onto $\bar{\Omega}$ (use Proposition 8.1). We claim that $M^{\prime} \sim \bar{v} M_{a}(u)$. Were this proved, (6.22) and the fact that $M_{a}(u)\llcorner\partial \Omega=0$ (recall that the trace of $u$ on $\partial \Omega$ takes values in $S^{k-1}$ ) would imply $M \sim \bar{V} M_{a}(u)\llcorner\Omega$, and we would conclude the proof using the retraction of $\bar{V}$ to $\Omega$. We prove the claim by showing that $M^{\prime}$ and $M_{a}(u)$ can be obtained as limits-with respect to the weak convergence of currents - of rectifiable currents which are cobordant in $\bar{V}$ (cf. Section 5.1).

By the choice of $a$, the current $\alpha_{k} M_{a}(u)$ is the limit of $\star J u_{a, \varepsilon}$ in the flat norm $\mathbf{F}_{\Omega^{\prime}}$, and $F_{\varepsilon}\left(u_{a, \varepsilon}, \Omega^{\prime}\right)=O(|\log \varepsilon|)$. Following the proof of the compactness statement in Proposition 3.1 (see Section 3), we see that the currents $\star J u_{a, \varepsilon}$ are asymptotically equivalent in the flat norm $\mathbf{F}_{\Omega^{\prime}}$ to $\star J_{\psi} u_{a, \varepsilon}$, where $\psi=\rho d y$ is a $k$-form with support contained in $B_{1 / 2}^{k}$ and integral $\alpha_{k}$, and $J_{\psi}$ is defined in Section 3.5. In turn, $\star J_{\psi} u_{a, \varepsilon}$ are asymptotically equivalent in the flat norm $\mathbf{F}_{V}$ to the polyhedral deformations $\Phi_{\varepsilon \sharp}\left(\star J_{\psi} u_{a, \varepsilon}\right)$, which are uniformly bounded in mass $\left(\Phi_{\varepsilon}\right.$ are the retractions associated to the grids $\mathcal{G}_{\varepsilon}$ as in Lemmas 3.8 and 3.9).

By Lemma 3.9(ii), $\Phi_{\varepsilon \sharp}\left(\star J_{\psi} u_{a, \varepsilon}\right)=\alpha_{k} \Phi_{\varepsilon \sharp}\left(M_{y}\left(u_{a, \varepsilon}\right)\right)$ for any regular value $y=y(\varepsilon)$ of $u_{a, \varepsilon}$ with $|y|<\frac{1}{2}$. Thus $M_{a}(u)$ is the limit in the flat norm $\mathbf{F}_{V}$ of the integral polyhedral currents $\Phi_{\varepsilon \sharp}\left(M_{y}\left(u_{a, \varepsilon}\right)\right)$, which are uniformly bounded in mass. Similarly, $M^{\prime}$ is the limit, in the flat norm $\mathbf{F}_{V}$, of the polyhedral currents $\Phi_{\varepsilon \sharp}\left(M_{y}\left(u_{\varepsilon}^{\prime}\right)\right.$ ), which are also uniformly bounded in mass (we take the same $y=$ $y(\varepsilon)$ as above).

\footnotetext{
${ }^{19} F_{\Omega^{\prime}}\left(T_{i}-T\right) \rightarrow 0$ means that there exist currents $S_{i}$ on $\Omega^{\prime}$ such that $\partial S_{i}=T_{i}-T$ in $\Omega^{\prime}$ and $\left\|S_{i}\right\| \rightarrow 0$. Clearly, if $S_{i}$ is regarded as a current on $\mathbb{R}^{n+k}, \partial S_{i}$ may have an additional part supported on $\partial \Omega^{\prime}$. However, if all current $T_{i}$ are supported on $\Omega$, a careful use of truncation and of the cone construction allows to modify each $S_{i}$ so that $\partial S_{i}=T_{i}-T$ in $\mathbb{R}^{n+k}$ (and $\left\|S_{i}\right\|$ still converge to 0 ).
} 
It remains to show that $\Phi_{\varepsilon \sharp}\left(M_{y}\left(u_{\varepsilon}^{\prime}\right)\right)$ and $\Phi_{\varepsilon \sharp}\left(M_{y}\left(u_{a, \varepsilon}\right)\right)$ are cobordant in $\bar{V}$. Since $u_{a, \varepsilon}$ and $u_{\varepsilon}^{\prime}$ agree outside $\Omega, M_{y}\left(u_{\varepsilon}^{\prime}\right)-M_{y}\left(u_{a, \varepsilon}\right)$ is supported in $\bar{\Omega}$, and by (5.2) is cobordant to 0 in $\Omega$. Hence $\Phi_{\varepsilon \sharp}\left(M_{y}\left(u_{\varepsilon}^{\prime}\right)-M_{y}\left(u_{a, \varepsilon}\right)\right)$ is cobordant to 0 in $\bar{V}$ for $\varepsilon$ small enough by a known property of polyhedral deformation, ${ }^{20}$ and this concludes the proof.

Let us point out a couple of technical issues in this second step of the proof. Firstly, the argument above, as most lemmas in Section 3, requires that $u_{\varepsilon}^{\prime}$ and $u_{a, \varepsilon}$ are smooth in $\Omega^{\prime}$. This can be fixed by regularizing these maps (e.g., by convolution). Indeed we never used that they have trace $v$ on $\partial \Omega$, but only that they agree outside $\bar{\Omega}$ (in fact, it is enough that they agree outside $\bar{V}$ ). Secondly, the argument works provided that the size of the grid $\mathcal{G}_{\varepsilon}$ is taken as in (3.22), and the center is chosen so that estimates (3.18)-(3.20) — but not (3.17)—hold for $u_{\varepsilon}^{\prime}$ and $u_{a, \varepsilon}$ at the same time. This can be achieved by a suitable modification of Lemma 3.11.

\section{Proof of Theorem 5.5(ii) ANd Corollary 5.6}

In this section we assume that the potential $W$ in the definition of $F_{\varepsilon}$ takes a constant value inside the unit ball of $\mathbb{R}^{k}$ : it is clear that proving statement (ii) of Theorem 5.5 for this particular choice of $W$ is sufficient. ${ }^{21}$ This assumption is used in the proof of Lemma 7.1 only.

For the rest, we follow the notation of Section 6. In particular, $n \geq 1$ and $\Omega$ is a bounded Lipschitz domain.

The proof of statement (ii) of Theorem 5.5 is a modification of the proof of statement (ii) of Theorem 1.1 given in Section 4 . Hence we need an $S^{k-1}$-valued map $\tilde{u}$ with the same properties as the one used in Section 4, which satisfies the additional condition $\tilde{u}=v$ on $\partial \Omega$. This refinement of Theorem 5.10 of [3] is proved in Theorem $\mathbf{9 . 6}$ for special boundary data $v$. In order to handle a general $v$, we paste together the maps $u_{\varepsilon}$ defined in (4.3) and the maps $u_{a, \varepsilon}$ defined in (6.7). This last step is based on the following lemma.

Lemma 7.1. Let $\Omega_{1}$ be a Lipschitz domain relatively compact in $\Omega$, and set $\Omega_{2}:=\Omega \backslash \bar{\Omega}_{1}$. Let maps $u_{i} \in W^{1, k}\left(\Omega_{i}, \mathbb{R}^{k}\right)$ for $i=1,2$ be given so that $\left|u_{1}\right|$, $\left|u_{2}\right| \leq 1$ everywhere and their traces on $\Sigma:=\partial \Omega_{1}$ (the interface between $\Omega_{1}$ and $\Omega_{2}$ ) satisfy the following compatibility condition:

$$
\left|u_{1}(x)\right|=\left|u_{2}(x)\right|=1 \Rightarrow u_{1}(x)=u_{2}(x) \quad \text { for } \mathcal{H}^{n+k-1} \text {-a.e. } x \in \Sigma .
$$

Then there exist positive finite constants $\eta_{0}$ and $C$ (depending on $\Omega$ and $\Omega_{1}$ ) so that, for every $0<\eta \leq \eta_{0}$ there exists $u \in W^{1, k}\left(\Omega, \mathbb{R}^{k}\right)$ which agrees with $u_{1}$ in $\Omega_{1}$ and

\footnotetext{
${ }^{20}$ More precisely, the deformation of the boundary of a current supported in $\bar{\Omega}$ is the boundary of a polyhedral current supported on a $d$-neighbourhood of $\Omega$, where $d$ is, say, twice the diameter of a cube in the grid (cf. [20, Theorem 4.2.9]).

${ }^{21} \mathrm{~W}$ is not continuous, but this is not needed in the construction that follows.
} 
with $u_{2}$ in $\partial \Omega$, and for every $\varepsilon>0$ satisfies

$$
F_{\varepsilon}\left(u, \Omega_{2}\right) \leq 2 F_{\varepsilon}\left(u_{2}, \Omega_{2}\right)+C \eta \int_{\Sigma} \frac{1}{\eta^{k}}+e_{\varepsilon}\left(u_{1}\right)+e_{\varepsilon}\left(u_{2}\right) .
$$

Proof. By Proposition 8.1, we can find a neighbourhood $U$ of $\partial \Omega_{1}=\Sigma$ which is relatively compact in $\Omega$ and homeomorphic to $(-1,1) \times \Sigma$ via some bi-Lipschitz map $\Psi$. For every $0<\eta<1$, we set $u:=u_{1}$ in $\Omega_{1}, u:=u_{2}$ in $\Omega_{2} \backslash U$, and $u:=u^{\prime} \circ \Psi^{-1}$ in $U \backslash \Omega_{1}=\Psi([0,1) \times \Sigma)$, where

$$
u^{\prime}\left(t, x^{\prime}\right):= \begin{cases}(1-t / \eta) u_{1}\left(x^{\prime}\right)+(t / \eta) u_{2}\left(x^{\prime}\right) & \text { for } 0 \leq t<\eta, \\ u_{2}\left(\Psi\left((t-\eta) /(1-\eta), x^{\prime}\right)\right) & \text { for } \eta \leq t<1 .\end{cases}
$$

Hence

$$
F_{\varepsilon}\left(u, \Omega_{2} \backslash U\right)=F_{\varepsilon}\left(u_{2}, \Omega_{2} \backslash U\right) .
$$

It remains to estimate the energy of $u$ in $U \cap \Omega_{2}$. To this end, we decompose $U \cap \Omega_{2}$ as $U_{\eta} \cup U_{\eta}^{\prime}$ with $U_{\eta}:=\Psi([0, \eta) \times \Sigma)$ and $U_{\eta}^{\prime}:=\Psi([\eta, 1) \times \Sigma)$. For almost every $\left(t, x^{\prime}\right) \in[0, \eta) \times \Sigma$ there holds

$$
\left|D u^{\prime}\left(t, x^{\prime}\right)\right|^{k} \leq K\left[\left|D u_{1}\left(x^{\prime}\right)\right|^{k}+\left|D u_{2}\left(x^{\prime}\right)\right|^{k}+\frac{1}{\eta^{k}}\right],
$$

while condition (7.1) and the fact that $W(y)$ is constant for $|y|<1$ yield

$$
W\left(u^{\prime}\left(t, x^{\prime}\right)\right) \leq W\left(u_{1}\left(x^{\prime}\right)\right)+W\left(u_{2}\left(x^{\prime}\right)\right) .
$$

Hence

$$
F_{\varepsilon}\left(u^{\prime},[0, \eta) \times \Sigma\right) \leq K \eta \int_{\Sigma} \frac{1}{\eta^{k}}+e_{\varepsilon}\left(u_{1}\right)+e_{\varepsilon}\left(u_{2}\right) .
$$

The change of variable formula gives the estimate

$$
F_{\varepsilon}(u, \Psi(A)) \leq C_{1} F_{\varepsilon}(u \circ \Psi, A),
$$

where $C_{1}:=\left(1 \vee \operatorname{Lip}\left(\Psi^{-1}\right)\right)^{k} \cdot \operatorname{Lip}(\Psi)^{n+k}$, and therefore

$$
F_{\varepsilon}\left(u, U_{\eta}\right) \leq C_{1} F_{\varepsilon}\left(u^{\prime},(0, \eta) \times \Sigma\right) \leq K C_{1} \eta \int_{\Sigma} \frac{1}{\eta^{k}}+e_{\varepsilon}\left(u_{1}\right)+e_{\varepsilon}\left(u_{2}\right) .
$$

By (8.2) and (7.3), for every $x \in U_{\eta}^{\prime}$ there holds $u(x)=u_{2}\left(R_{\eta}(x)\right)$, where

$$
R_{\eta}(x):=x-\eta \frac{1-t}{1-\eta} v\left(x^{\prime}\right),
$$


and $t$, $x^{\prime}$ satisfy $x=x^{\prime}+t v(x)=\Psi\left(t, x^{\prime}\right)$. Hence $\operatorname{Lip}\left(R_{\eta}\right)$ and $\operatorname{Lip}\left(R_{\eta}^{-1}\right)$ are both of order $1+O(\eta)$, and formula (7.5) yields

$$
F_{\varepsilon}\left(u, U_{\eta}^{\prime}\right) \leq(1+O(\eta)) \cdot F_{\varepsilon}\left(u_{2}, U \backslash \Omega_{1}\right) .
$$

For $\eta$ small enough, estimates (7.4), (7.6), (7.7) yield (7.2).

Proof of statement (ii) of Theorem 5.5. Let $u$ be a map in $W^{1, k}\left(\Omega, \mathbb{R}^{k}\right)$ with trace $v$ on $\partial \Omega$ such that $|u| \leq 1$ and $u$ is locally piecewise affine in $\Omega$, that is, agrees with some piecewise affine map on every compact subset of $\Omega$ (such a map can be obtained from any $u$ with trace $v$ by truncation and a local regularization argument à la Meyers-Serrin).

First reduction. A simple diagonal argument shows that the statement we want to prove is equivalent to the following: for every $\delta>0$, every admissible current $M$ and every countable subsequence of $(\varepsilon)$ there exists a subsequence (not relabelled) for which we can construct maps $u_{\varepsilon}$ such that $\star J u_{\varepsilon}$ converge to $\alpha_{k} M$ and

$$
\limsup \frac{F_{\varepsilon}\left(u_{\varepsilon}, \Omega\right)}{|\log \varepsilon|} \leq \beta_{k}\|M\|+\delta .
$$

This remark allows us to pass freely to subsequences in the construction below.

Let $\delta$ and the countable subsequence of $(\varepsilon)$ be fixed. We take now $d>$ 0 so that $\int_{U_{d}}|D u|^{k} \leq \delta^{2} /(2 K)$, where $U_{d}$ is the set of all $x \in \Omega$ such that $\operatorname{dist}(x, \partial \Omega)<d$ and $K$ is the universal constant in (6.11). Then, by Proposition 6.4(iv) we can find $y \in B_{1}^{k}$ so that, passing to a subsequence, the maps $u_{y, \varepsilon}$ given in (6.7) satisfy

$$
\lim _{\varepsilon \rightarrow 0} \frac{F_{\varepsilon}\left(u_{y, \varepsilon}, U_{d}\right)}{|\log \varepsilon|} \leq \frac{K}{\delta} \int_{U_{d}}|D u|^{k} \leq \frac{\delta}{2} .
$$

Second reduction. A simple diagonal argument and Proposition 8.7 show that it suffices to prove the claim above when the current $M$ has multiplicity 1 and can be written as $M=M_{y}(u)+\partial N$, where $N$ is a polyhedral current of dimension $n+1$ with compact support in $\Omega$.

Let $M$ and $N$ be fixed, and for every $\varepsilon>0$, let a positive $\eta=\eta(\varepsilon)$ be giventhe correct choice of $\eta$ will be specified below. Now choose a polyhedral domain $\Omega_{d}$ so that $N \Subset \Omega_{d} \Subset \Omega, \Omega_{d} \cup U_{d}=\Omega$ (see Figure 7.1 below) and

$$
\liminf _{\varepsilon \rightarrow 0} \frac{1}{|\log \varepsilon|} \int_{\partial \Omega_{d}} \frac{1}{\eta^{k}}+e_{\varepsilon}\left(u_{y, \varepsilon}\right) \leq \frac{\delta}{2 d}+\frac{|\Omega|}{\eta^{k}|\log \varepsilon|} .
$$

To find such $\Omega_{d}$, apply Lemma 8.5 with $g: U_{d} \rightarrow[0, d / 2]$ a piecewise affine 1-Lipschitz function such that $g=0$ on $\partial \Omega$ and $g=d / 2$ on $\partial U_{d} \backslash \Omega$-such 


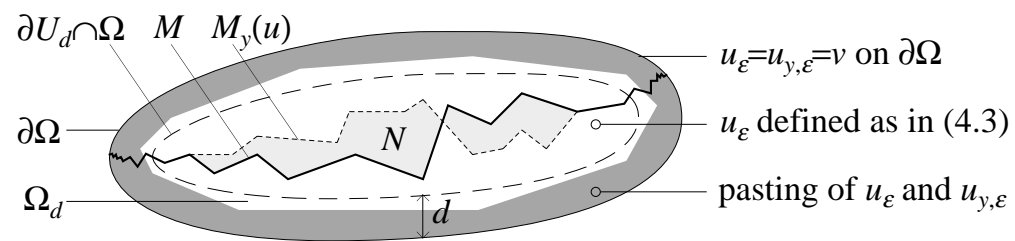

FIGURE 7.1.

a function exists because the distance between these two sets is $d$-and then use estimate (7.9). After passing to a subsequence, we may assume that the liminf at the left-hand side of (7.10) is a limit.

By Theorem 9.6, there exists a map $\tilde{u}: \bar{\Omega}_{d} \rightarrow S^{k-1}$ which satisfies assumptions (i), (ii), (iii) of Lemma 4.2 with $\Omega$ replaced by $\Omega_{d}$, and $\tilde{u}=u_{y}$ on $\partial \Omega_{d}$, where $u_{y}$ is defined as in (6.7). Take $u_{\varepsilon}: \bar{\Omega}_{d} \rightarrow \mathbb{R}^{k}$ as in (4.3): then the Jacobians $\star J u_{\varepsilon}$ converge in the sense of currents to $\alpha_{k} M$ on $\Omega_{d}$, and

$$
\limsup _{\varepsilon \rightarrow 0} \frac{F_{\varepsilon}\left(u_{\varepsilon}, \Omega_{d}\right)}{|\log \varepsilon|} \leq \beta_{k}\|M\|_{\Omega_{d}} \leq \beta_{k}\|M\|_{\Omega} .
$$

Moreover, as pointed out in Remark 4.3,

$$
\int_{\partial \Omega_{d}} e_{\varepsilon}\left(u_{\varepsilon}\right)=O(|\log \varepsilon|) .
$$

It is easy to check that $u_{\varepsilon}$ and $u_{y, \varepsilon}$ satisfy the compatibility condition (7.1) on $\partial \Omega_{d}$, and therefore we can apply Lemma 7.1 to extend the maps $u_{\varepsilon}$ to $\Omega$ so that they agree with $u_{y, \varepsilon}=v$ on $\partial \Omega$. Moreover, estimate (7.2) yields

$$
\begin{aligned}
F_{\varepsilon}\left(u_{\varepsilon}, \Omega\right) \leq F_{\varepsilon} & \left(u_{\varepsilon}, \Omega_{d}\right)+2 F_{\varepsilon}\left(u_{y, \varepsilon}, \Omega \backslash \bar{\Omega}_{d}\right) \\
& +C \eta \int_{\partial \Omega_{d}} \frac{1}{\eta^{k}}+e_{\varepsilon}\left(u_{\varepsilon}\right)+e_{\varepsilon}\left(u_{y, \varepsilon}\right) .
\end{aligned}
$$

Now we divide both sides of (7.13) by $|\log \varepsilon|$ and take the limsup as $\varepsilon \rightarrow 0$. If we have chosen $\eta=\eta(\varepsilon)$ so that it tends to 0 and $1 / \eta=o\left(|\log \varepsilon|^{1 /(k-1)}\right)$, then estimates (7.10) and (7.12) imply that the term in the second line of formula (7.13) is negligible with respect to $|\log \varepsilon|$, and recalling estimates (7.9) and (7.11) we obtain (7.8).

It remains to prove that the Jacobians $\star J u_{\varepsilon}$ converge to $\alpha_{k} M$ in the flat norm $\mathbf{F}_{\mathbb{R}^{n+k}}$. On the one hand, we already know that $\star J u_{\varepsilon}$ converge in the sense of currents to $\alpha_{k} M$ on every $\Omega_{d}$, and therefore also on $\Omega$. On the other hand, by estimate (7.8) and statement (i) of Theorem 5.5, $\star J u_{\varepsilon}$ converge, up to a subsequence and in the flat norm $\mathbf{F}_{\mathbb{R}^{n+k}}$, to some $\alpha_{k} M^{\prime}$, and

$$
\beta_{k}\left\|M^{\prime}\right\|_{\mathbb{R}^{n+k}} \leq \liminf _{\varepsilon \rightarrow 0} \frac{F_{\varepsilon}\left(u_{\varepsilon}, \Omega\right)}{|\log \varepsilon|} .
$$


It remains to prove that $M^{\prime}=M$. Note that $M$ is supported in $\Omega$ and agrees with $M^{\prime}$ in $\Omega$, that is, $M=M^{\prime}\llcorner\Omega$. Combining (7.8) and (7.14) we obtain $\left\|M^{\prime}\right\| \leq\|M\|$, and therefore $M^{\prime}$ must be equal to $M$.

Proof of Corollary 5.6. Estimate (5.5) follows by applying statement (ii) of Theorem 5.5 to any admissible $M$ with finite mass-for instance any regular level set $M_{y}(u)$ of any map $u \in W^{1, k}\left(\Omega, \mathbb{R}^{k}\right)$ with trace $v$ on $\partial \Omega$.

Let $\left(u_{\varepsilon}\right)$ be a sequence of maps that satisfy (5.6). Then $F_{\varepsilon}\left(u_{\varepsilon}\right)=O(|\log \varepsilon|)$, and Theorem 5.5(i) implies that $\star J u_{\varepsilon}$ converge, up to a subsequence and in the flat norm $\mathbf{F}_{\mathbb{R}^{n+k}}$, to $\alpha_{k} M$, where $M$ is a rectifiable current cobordant to $M_{y}$ in $\bar{\Omega}$, and $\beta_{k}\|M\| \leq \liminf |\log \varepsilon|^{-1} F_{\varepsilon}\left(u_{\varepsilon}\right)$.

On the other hand, by Theorem 5.5(ii), every $M^{\prime}$ such that $M^{\prime} \sim \bar{\Omega} M_{y}$ can be realized as a limit of Jacobians $\star J u_{\varepsilon}^{\prime}$ so that $\lim |\log \varepsilon|^{-1} F_{\varepsilon}\left(u_{\varepsilon}^{\prime}\right)=\beta_{k}\left\|M^{\prime}\right\|$. Hence the minimizing property (5.6) implies $\|M\| \leq\left\|M^{\prime}\right\|$ (and therefore $M$ is mass-minimizing) and $\lim |\log \varepsilon|^{-1} F_{\varepsilon}\left(u_{\varepsilon}\right)=\beta_{k}\|M\|$. As pointed out in Remark (iii) after Theorem 5.5, the latter condition implies (1.5).

Let $\lambda$ be any limit point (in the sense of measures on $\mathbb{R}^{n+k}$ ) of the rescaled energy densities $\lambda_{\varepsilon}:=|\log \varepsilon|^{-1} e_{\varepsilon}\left(u_{\varepsilon}\right)$, extended to 0 outside $\Omega$. By Theorem 5.5(i), for every open set $A \subset \mathbb{B}^{n+k}$ there holds

$$
\beta_{k}|M|(A) \leq \liminf _{\varepsilon \rightarrow 0} \int_{A \cap \Omega} \lambda_{\varepsilon} \leq \lambda(\bar{A}) .
$$

Thus $\beta_{k}|M|(A) \leq \lambda(A)$ when $A$ is an open set such that $|\lambda|(\partial A)=0$, and by approximation also when $A$ is a Borel set. On the other hand, $\|\lambda\| \leq \lim \left\|\lambda_{\varepsilon}\right\|=$ $\beta_{k}\|M\|$, and therefore $\lambda=\beta_{k}|M|$. In other words, the rescaled energy densities $|\log \varepsilon|^{-1} e_{\varepsilon}\left(u_{\varepsilon}\right)$ converge to $\beta_{k}|M|$ in the sense of measures on $\mathbb{R}^{n+k}$. The same holds for $|\log \varepsilon|^{-1}\left|D u_{\varepsilon}\right|^{k} / k$ because of (1.5).

\section{Appendix A: Technical lemmas and Approximation Results}

Proposition 8.1. Let $\Omega$ be a bounded Lipschitz domain in $\mathbb{R}^{d}$. Then there exists a bi-Lipschitz homeomorphism $\Psi$ which maps the product $(-1,1) \times \partial \Omega$ onto a neighbourhood $U$ of $\partial \Omega$. Moreover, $\Psi$ takes $(-1,0) \times \partial \Omega$ onto $U \cap \Omega$ and $[0,1) \times \partial \Omega$ onto $U \backslash \Omega$, and $\Psi(0, x)=x$ for every $x \in \partial \Omega$.

Proof. For every $x \in \partial \Omega$ and $\varepsilon>0$, denote by $C(x)$ the set of all vectors $v \in \mathbb{R}^{d}$ such that $x+\lambda v \in \mathbb{R}^{d} \backslash \bar{\Omega}$ and $x-\lambda v \in \Omega$ for every $\lambda \in(0,1]$ (thus $v \neq 0)$, and by $C_{\varepsilon}(x)$ the set of all $v$ such that $v+B_{\varepsilon} \subset C(x)$.

Assume $x \in \partial \Omega$, vectors $v, v^{\prime} \in \mathbb{R}^{d}$ and $r>0$ such that $|v|,\left|v^{\prime}\right| \leq r$ and $v, v^{\prime} \in C\left(x^{\prime}\right)$ for every $x^{\prime} \in\left(x+B_{r}\right) \cap \partial \Omega$. Then every convex combination of $v$ and $v^{\prime}$ belongs to $C(x)$. Let indeed $T^{+}$and $T^{-}$be the triangles with vertices $x$, $x+v, x+v^{\prime}$ and $x, x-v$ and $x-v^{\prime}$, respectively. It is easy to check that $T^{+}$and $T^{-}$intersect $\partial \Omega$ only in the common vertex $x$, and therefore they are contained, except for $x$, in $\mathbb{R}^{d} \backslash \bar{\Omega}$ and $\Omega$, respectively. 
From the previous remark we deduce the following property: given $\varepsilon, r>0$ and a set $E \subset \mathbb{R}^{d}$, and denoting by $\operatorname{Conv}(E)$ the convex hull of $E$,

$$
E \subset C_{\varepsilon}\left(x^{\prime}\right) \cap \bar{B}_{r} \forall x^{\prime} \in\left(x+B_{r}\right) \cap \partial \Omega \Rightarrow \operatorname{Conv}(E) \subset C_{\varepsilon}(x) .
$$

The fact that $\Omega$ is a bounded Lipschitz domain means that there exist $\varepsilon>0$, finitely many open sets $U_{i}$ which cover $\partial \Omega$, and vectors $v_{i} \in \bar{B}_{1}$ such that $v_{i} \in$ $C_{\varepsilon}\left(x_{i}\right)$ for every $x \in U_{i}$. We take non-negative smooth functions $\varphi_{i}: \mathbb{R}^{d} \rightarrow$ $[0,1]$ with compact support in $U_{i}$ such that $\sum \varphi_{i}=1$ on $\partial \Omega$, and denote by $r$ the minimum of $\operatorname{dist}\left(\operatorname{supp}\left(\varphi_{i}\right), \partial U_{i}\right)$ over all $i$. Then the map $v(x):=r \sum \varphi_{i}(x) v_{i}$ is smooth and compactly supported, and (8.1) implies $v(x) \in C_{\varepsilon}(x)$ for every $x \in \partial \Omega$, and in particular $v(x) \neq 0$. We set

$$
\Psi(t, x):=x+t v(x) \quad \text { for every } t \in \mathbb{R}, x \in \mathbb{R}^{d} .
$$

Thus $\Psi$ is smooth, $\Psi(0, x)=x$ for every $x \in \partial \Omega$, and the linear map $D \Psi(0, x)$ takes $(d t, d x) \in \mathbb{R} \times \mathbb{R}^{d}$ in $v(x) d t+d x$. Then the kernel of $D \Psi(0, x)$ is spanned by the vector $(1,-v(x))$.

We claim that there exists $\delta>0$ such that the restriction of $\Psi$ to the set $(-\delta, \delta) \times \partial \Omega$ is bi-Lipschitz. This would also imply that $\Psi$ is open on this set, and conclude the proof. Were the claim not true, we could find sequences $\left(t_{i}, x_{i}\right)$ and $\left(t_{i}^{\prime}, x_{i}^{\prime}\right)$ in $\mathbb{R} \times \partial \Omega$, converging to $(0, x)$ and $\left(0, x^{\prime}\right)$, respectively, such that $\left(t_{i}, x_{i}\right) \neq\left(t_{i}^{\prime}, x_{i}^{\prime}\right)$ and

$$
\frac{\left|\Psi\left(t_{i}, x_{i}\right)-\Psi\left(t_{i}^{\prime}, x_{i}^{\prime}\right)\right|}{\left|\left(t_{i}-t_{i}^{\prime}, x_{i}-x_{i}^{\prime}\right)\right|} \rightarrow 0
$$

If $x \neq x^{\prime}$, (8.3) implies $0=\Psi(0, x)-\Psi\left(0, x^{\prime}\right)=x-x^{\prime}$, which is a contradiction. If $x=x^{\prime}$, (8.3) implies that every limit point of every renormalized sequence $\lambda_{i}\left(t_{i}-t_{i}^{\prime}, x_{i}-x_{i}^{\prime}\right)$ is in the kernel of $D \Psi(0, x)$, and can be written as $\lambda(1,-v(x))$ for some $\lambda \in \mathbb{R}$. In particular we can find $\lambda_{i}$ so that $\lambda_{i}\left(x_{i}-x_{i}^{\prime}\right)$ converge to $v(x)$. On the other hand, $x_{i}$ and $x_{i}^{\prime}$ belong to $\partial \Omega$, and since $v\left(x_{i}\right) \in C_{r \varepsilon}\left(x_{i}\right)$, the angle between $x_{i}^{\prime}-x_{i}$ and $v\left(x_{i}\right)$ is at least $\arcsin \left(r \varepsilon /\left|v\left(x_{i}\right)\right|\right)$ for $i$ large enough. Since the map $v$ is continuous, the same is true, in the limit, for the angle between $x_{i}^{\prime}-x_{i}$ and $v(x)$, and this contradicts the previous conclusion.

Remark 8.2. Let $\Psi$ be taken as in Proposition 8.1, and $R$ be the reflection map on $(-1,1) \times \partial \Omega$, namely $R(t, x):=(-t, x)$. Then $\Phi:=\Psi \circ R \circ \Psi^{-1}$ is a bi-Lipschitz map of $U$ onto itself which agrees with the identity on $\partial \Omega$, and swaps $U \cap \bar{\Omega}$ with $U \backslash \Omega$, that is, a reflection with respect to $\partial \Omega$.

Proof of Proposition 2.6. Let $V$ be a smooth bounded domain such that $U \Subset$ $V \subset \Omega$. By the isoperimetric theorem, for every rectifiable boundary $M$ in $V$ with finite mass there exists an integral current $N$ in $V$ such that $M=\partial N$ and 
$\|N\|_{V} \leq C\|M\|_{V}^{1+1 / n}$, where the constant $C$ depends only on $V$ (apply Theorem 4.4.2(2) in [20] with $A:=\bar{V}, B:=\partial V$, and $U, W$ neighbourhoods of $A$ and $B$ that admit a Lipschitz retraction on $A$ and $B$, respectively).

Thus we can find integral currents $N_{i}$ in $V$ which are uniformly bounded in mass and satisfy $M_{i}=\partial N_{i}$, and by the closure theorem for integral currents (cf. [39, Theorem 27.3 and Remark 31.3]), we can extract a subsequence $N_{j}$ which converges to an integral current $N$ in the flat metric topology of $V$, as defined in [39, Section 31]. This immediately implies the convergence of the corresponding boundaries $M_{j}$ to $M:=\partial N$ in $\mathbf{F}_{U}$.

Lemma 8.3. Let $S \subset \mathbb{R}^{d}$ be a bounded set contained in a finite union of Lipschitz surfaces of codimension $h$, and for every $t>0$, denote by $S_{t}$ the $t$-neighbourhood of $S$. There exists a finite constant $C$ (depending on $S$ ) such that $\mathcal{L}^{d}\left(S_{t}\right) \leq C t^{h}$ for every $t \geq 0$ and

$$
\begin{aligned}
\int_{S_{t}} \frac{d x}{[\operatorname{dist}(x, S)]^{p}} \leq \frac{C}{h-p} t^{h-p} & \text { for every } p<h \text { and } t \geq 0, \\
\int_{S_{t} \backslash S_{t^{\prime}}} \frac{d x}{[\operatorname{dist}(x, S)]^{h}} \leq C \log \frac{t}{t^{\prime}} & \text { for every } t \geq t^{\prime} \geq 0 .
\end{aligned}
$$

Proof. The estimate on $\mathcal{L}^{d}\left(S_{t}\right) \leq C t^{h}$ follows from [20, Section 3.2.39]. For every $t>0$ denote by $1_{t}$ the characteristic function of $S_{t}$. Hence

$$
\frac{1}{[\operatorname{dist}(x, S)]^{p}}=t^{-p}+p \int_{0}^{t} \frac{1_{\tau}(x)}{\tau^{p+1}} d \tau \quad \text { for every } x \in S_{t},
$$

and

$$
\int_{S_{t}} \frac{d x}{[\operatorname{dist}(x, S)]^{p}}=\mathcal{L}^{d}\left(S_{t}\right) t^{-p}+p \int_{0}^{t} \frac{\mathcal{L}^{d}\left(S_{\tau}\right)}{\tau^{p+1}} d \tau \leq \frac{C h}{h-p} t^{h-p} .
$$

A similar computation yields the rest of the statement.

Lemma 8.4. Let $X$ be a measurable space endowed with a finite measure $\mu$. Given positive measurable functions $f_{0}, \ldots, f_{m}$ on $X$ and $\delta>0$, there exists $a \in X$ such that

$$
\begin{gathered}
f_{0}(a) \leq(1+\delta) f_{X} f_{0} d \mu, \\
f_{i}(a) \leq(1+\delta) \frac{m}{\delta} f_{X} f_{i} d \mu \quad \text { for } i=1, \ldots, m .
\end{gathered}
$$

Proof. We can assume that $\mu$ is a probability measure. Let $E_{i}$ be the set of all $a$ where $f_{i}(a)$ fails to fulfil the required inequality. Then $\mu\left(E_{0}\right)<1 /(1+\delta)$, while $\mu\left(E_{i}\right)<\delta /(m(1+\delta))$ for $i=1, \ldots, m$. Hence the measure of the set $E_{0} \cup \cdots \cup E_{m}$ is less than 1, which implies that the complement is not empty. 
Lemma 8.5. Let $\Omega$ be an open subset of $\mathbb{R}^{d}$. Given a 1-Lipschitz function $g$ on $\Omega$, denote by $M$ and $m$ respectively the supremum and the infimum of $g$ on $\Omega$, and by $\Omega_{t}$ the $t$-sublevel set of $g$. Then, for every sequence of positive Borel functions $f_{\varepsilon}$ on $\Omega$ there exists $t$ such that $m<t<M$ and

$$
\liminf _{\varepsilon \rightarrow 0} \int_{\partial \Omega_{t}} f_{\varepsilon} d \mathcal{H}^{d-1} \leq \frac{1}{M-m} \liminf _{\varepsilon \rightarrow 0} \int_{\Omega} f_{\varepsilon} d \mathcal{L}^{d} .
$$

Proof. Since $|D g| \leq 1$, the coarea formula for Lipschitz functions yields, for every $\varepsilon>0$,

$$
\int_{m}^{M}\left[\int_{\partial \Omega_{t}} f_{\varepsilon} d \mathcal{H}^{d-1}\right] d t=\int_{\Omega} f_{\varepsilon}|D g| d \mathcal{L}^{d} \leq \int_{\Omega} f_{\varepsilon} d \mathcal{L}^{d},
$$

and by Fatou's lemma,

$$
\int_{m}^{M}\left[\liminf _{\varepsilon \rightarrow 0} \int_{\partial \Omega_{t}} f_{\varepsilon} d \mathcal{H}^{d-1}\right] d t \leq \liminf _{\varepsilon \rightarrow 0} \int_{\Omega} f_{\varepsilon} d \mathcal{L}^{d} .
$$

To conclude, it suffices to choose $t$ properly.

We conclude this appendix with two approximation results for integral currents.

Proposition 8.6. Let $\Omega$ be a bounded Lipschitz domain in $\mathbb{R}^{d}, d \geq 2$, and let $M$ be an integral boundary in $\Omega$ with dimension $h<d$ and finite mass. Then

(i) $M$ agrees (in $\Omega$ ) with the boundary of an integral $(h+1)$-current $N$ in $\mathbb{R}^{d}$ with compact support such that $|\partial N|(\partial \Omega)=0$;

(ii) $M$ can be approximated in the flat norm $\mathbf{F}_{\Omega}$ by a sequence of polyhedral boundaries $M_{i}$ in $\mathbb{R}^{d}$ so that $\left\|M_{i}\right\|_{\Omega} \rightarrow\|M\|_{\Omega}$. Moreover, we can require that $\left|M_{i}\right|(\partial \Omega)=0$ and $M_{i}$ has multiplicity 1 for every $i$.

Proof. Let $M=\partial N$, where $N$ is an integral current $N$ in $\Omega$.

We prove statement (i) first. We extend $N$ to a neighbourhood of $\bar{\Omega}$ using the reflection map $\Phi$ in Remark 8.2. More precisely, we set $\Omega^{\prime}:=\Omega \cup U$, where $U$ is the tubular neighbourhood in Proposition 8.1. Then the boundary of $N$ in $\Omega^{\prime}$ is given by $M+T$, where $T$ is supported on $\partial \Omega(\|T\|$ is not necessarily finite). If we set $N^{\prime}:=N-\Phi_{\sharp} N$, then $\partial N^{\prime}=M-\Phi_{\sharp} M$ in $\Omega^{\prime} .{ }^{22}$ Hence $N^{\prime}$ is an integral current in $\Omega^{\prime}$ which agrees with $N$ in $\Omega$, and $\left|\partial N^{\prime}\right|(\partial \Omega)=0$. Finally, a suitable truncation makes $N^{\prime}$ an integral current in $\mathbb{R}^{d}$.

We prove now statement (ii). By statement (i), we can assume that $M=\partial N$, where $N$ is an integral current in $\mathbb{R}^{d}$ with compact support, and $|\partial N|(\partial \Omega)=0$. By standard approximation results (see, e.g., [20, Corollary 4.2.21]), we can find polyhedral currents $N_{i}$ which converge to $N$ in the usual flat norm for integral

\footnotetext{
${ }^{22}$ This can be verified using the identification of $U$ with $(-1,1) \times \partial \Omega$ provided by Proposition 8.1, and the explicit formula for the reflection map given in Remark 8.2.
} 
currents and $\left\|N_{i}\right\|+\left\|\partial N_{i}\right\| \rightarrow\|N\|+\|\partial N\|$ (where all masses are computed on the entire $\left.\mathbb{R}^{d}\right)$. It follows immediately that $\mathbf{F}_{\mathbb{R}^{d}}\left(\partial N_{i}-\partial N\right) \rightarrow 0$ and $\left\|\partial N_{i}\right\| \rightarrow$ $\|\partial N\|$, and the latter implies $\left\|\partial N_{i}\right\|_{U} \rightarrow\|\partial N\|_{U}$ for every open set $U$ such that $|\partial N|(\partial U)=0$, and in particular for $\left\|\partial N_{i}\right\|_{\Omega} \rightarrow\|\partial N\|_{\Omega}$.

We conclude the proof of statement (ii) with two simple remarks that allow us to choose $N_{i}$ so that $\left|\partial N_{i}\right|(\partial \Omega)=0$ and $N_{i}$ has multiplicity 1 . Firstly, if a polyhedral current $N$ does not satisfy $|\partial N|(\partial \Omega)=0$, yet generic translations do. Indeed, since $\partial \Omega$ has null Lebesgue measure, for every $n$-plane $P$ in $\mathbb{R}^{d}$, the set of all vectors $v \in \mathbb{R}^{d}$ such that $\mathcal{H}^{n}((v+P) \cap \partial \Omega)>0$ must be Lebesgue negligible by Fubini's theorem, and clearly the same holds if $P$ is a subset of a finite union of $n$-planes.

Secondly, every integral polyhedral boundary $M$ can be approximated in the flat norm by polyhedral boundaries with multiplicity 1 . Write $M$ as a finite sum $M=\sum \sigma_{i} F_{i}$, where each $F_{i}$ is an oriented simplex with multiplicity 1 and $\sigma_{i} \geq$ 1 an integer, and replace it by $M^{\prime}:=M+\sum_{i j} \partial G_{i j}$ where $j$ runs from 1 to $\sigma_{i}$ and each $G_{i j}$ is the cylinder which joins $F_{i}$ and a translation $F_{i}+v_{i j}$ (more precisely, the push-forward of the product current $[0,1] \times F_{i}$ in $\mathbb{R} \times \mathbb{R}^{d}$ according to the map $\left.(t, x) \mapsto\left(x+t v_{i j}\right)\right)$. Thus $M^{\prime}$ is polyhedral and cobordant to $M$, it has multiplicity 1 for almost every choice of the vectors $v_{i j}$, and the distance $\mathbf{F}_{\mathbb{R}^{d}}\left(M-M^{\prime}\right)$ is bounded by $\sum_{i j}\left\|G_{i j}\right\| \leq \sum_{i j}\left|v_{i j}\right| \cdot\left\|F_{i}\right\|$ and therefore can be taken arbitrarily small.

Proposition 8.7. Let $\Omega$ be a bounded Lipschitz domain in $\mathbb{R}^{d}, d \geq 2$. Let $M^{\prime}$ be a rectifiable current supported in $\bar{\Omega}$ with dimension $h<d$ such that $\left|M^{\prime}\right|(\partial \Omega)=0$, and let $M$ be a rectifiable current supported in $\bar{\Omega}$ of the form $M=M^{\prime}+\partial N$, with $N$ an integral current supported in $\partial \Omega$.

The current $M$ can be approximated in the flat norm $\mathbf{F}_{\mathbb{R}^{d}}$ by a sequence of currents $M_{\varepsilon}$ of the form $M_{\varepsilon}=M^{\prime}+\partial N_{\varepsilon}$, where $N_{\varepsilon}$ is an integral current with compact support in $\Omega$, and $\left\|M_{\varepsilon}\right\| \rightarrow\|M\|$. If in addition $M^{\prime}$ has multiplicity 1 and agrees on every compact subset of $\Omega$ with a polyhedral current, then we can require that $N_{\varepsilon}$ is polyhedral, and $M_{\varepsilon}$ has multiplicity 1.

Proof. Take $U$ and $\Psi:(-1,1) \times \partial \Omega \rightarrow U$ as in Proposition 8.1. For every $0<\varepsilon<1$ we set $U_{\varepsilon}:=\Psi((-\varepsilon, \varepsilon) \times \partial \Omega)$, and take the following retraction of $\Omega \cup U_{\varepsilon}$ onto $\Omega \backslash U_{\varepsilon}$ :

$$
P_{\varepsilon}(x):= \begin{cases}x & \text { if } x \in \Omega \backslash U_{\varepsilon} \\ \Psi\left(P_{\varepsilon}^{\prime}\left(\Psi^{-1}(x)\right)\right) & \text { if } x \in U_{\varepsilon}\end{cases}
$$

where $P_{\varepsilon}^{\prime}\left(t, x^{\prime}\right):=\left(-\varepsilon, x^{\prime}\right)$ for every $\left(t, x^{\prime}\right) \in(-\varepsilon, \varepsilon) \times \partial \Omega$. We set

$$
N_{\varepsilon}:=P_{\varepsilon \#}(N) \quad \text { and } \quad M_{\varepsilon}:=M^{\prime}+\partial N_{\varepsilon} .
$$


Clearly, $\operatorname{supp}\left(N_{\varepsilon}\right) \subset \Omega$. Let us verify that $\mathbf{F}_{\mathbb{R}^{d}}\left(M_{\varepsilon}-M\right) \rightarrow 0$. To this end, we define the following homotopy between $P_{\varepsilon}$ and the identity map:

$$
R_{\varepsilon}(\lambda, x):= \begin{cases}x & \text { if } \lambda \in[0, \varepsilon] \text { and } x \in \Omega \backslash U_{\varepsilon}, \\ \Psi\left(R_{\varepsilon}^{\prime}\left(\lambda, \Psi^{-1}(x)\right)\right) & \text { if } \lambda \in[0, \varepsilon] \text { and } x \in U_{\varepsilon},\end{cases}
$$

where $R_{\varepsilon}^{\prime}\left(\lambda, t, x^{\prime}\right):=\left(-\lambda+(1-\lambda / \varepsilon) t, x^{\prime}\right)$ for $\left(\lambda, t, x^{\prime}\right) \in[0, \varepsilon] \times(-\varepsilon, \varepsilon) \times \partial \Omega$. Thus $R_{\varepsilon}(0, x)=x$ and $R_{\varepsilon}(\varepsilon, x)=P_{\varepsilon}(x)$. We extend $R_{\varepsilon}$ to all $\lambda \notin[0, \varepsilon]$ by setting it equal to $x$ for $\lambda<0$ and to $P_{\varepsilon}(x)$ for $\lambda>\varepsilon$. Using definition (8.4), and denoting by $I_{\varepsilon}$ the current associated to the oriented segment $[0, \varepsilon]$, we obtain

$$
\begin{aligned}
M_{\varepsilon}-M & =P_{\varepsilon \sharp}(\partial N)-\partial N=R_{\varepsilon \sharp}\left(\delta_{\varepsilon} \times \partial N-\delta_{0} \times \partial N\right) \\
& =R_{\varepsilon \sharp}\left(\partial I_{\varepsilon} \times \partial N\right)=R_{\varepsilon \sharp}\left(\partial\left(I_{\varepsilon} \times \partial N\right)\right)=\partial R_{\varepsilon \sharp}\left(I_{\varepsilon} \times\left(M-M^{\prime}\right)\right),
\end{aligned}
$$

and since $\operatorname{Lip}\left(R_{\varepsilon}\right)$ is bounded by a constant $C$ independent of $\varepsilon$,

$$
\begin{aligned}
\mathbf{F}_{\mathbb{R}^{n+k}}\left(M_{\varepsilon}-M\right) & \leq\left\|R_{\varepsilon \sharp}\left(I_{\varepsilon} \times\left(M-M^{\prime}\right)\right)\right\| \\
& \leq C^{h+1}\left\|I_{\varepsilon} \times\left(M-M^{\prime}\right)\right\| \leq C^{h+1} \varepsilon\left\|M-M^{\prime}\right\| .
\end{aligned}
$$

We prove now that $\left\|M_{\varepsilon}\right\|$ converge to $\|M\|$. Using the identity $M=M^{\prime}+\partial N$ and definition (8.4), and recalling that $P_{\varepsilon}$ agrees with the identity on $\Omega \backslash U_{\varepsilon}$, one easily verify that

$$
\begin{aligned}
M_{\varepsilon}= & M^{\prime}-P_{\varepsilon \sharp} M^{\prime}+\partial P_{\varepsilon \sharp}(M) \\
= & M^{\prime}\left\llcorner U_{\varepsilon}-P_{\varepsilon \sharp}\left(M^{\prime}\left\llcorner U_{\varepsilon}\right)+M\left\llcorner\left(\Omega \backslash U_{\varepsilon}\right)\right.\right.\right. \\
& \quad+P_{\varepsilon \sharp}\left(M\left\llcorner\left(U_{\varepsilon} \backslash \partial \Omega\right)\right)+P_{\varepsilon \sharp}(M\llcorner\partial \Omega) .\right.
\end{aligned}
$$

The assumption $\left|M^{\prime}\right|(\partial \Omega)=0$ implies that $\| M^{\prime}\left\llcorner U_{\varepsilon} \|=|M|\left(U_{\varepsilon}\right)\right.$ tends to 0 as $\varepsilon \rightarrow 0$, and the same holds for $\| P_{\varepsilon \#}\left(M^{\prime}\left\llcorner U_{\varepsilon}\right) \|\right.$ because the Lipschitz constants of the maps $P_{\varepsilon}$ are bounded independently of $\varepsilon$. Similarly, $\left\|M^{\prime} L\left(U_{\varepsilon} \backslash \partial \Omega\right)\right\|=$ $|M|\left(U_{\varepsilon} \backslash \partial \Omega\right)$ tends to 0 , and therefore the same holds for $\| P_{\varepsilon \sharp}\left(M^{\prime}\left\llcorner\left(U_{\varepsilon} \backslash \partial \Omega\right)\right) \|\right.$.

Obviously $\| M\left\llcorner\left(\Omega \backslash U_{\varepsilon}\right) \|=|M|\left(\Omega \backslash U_{\varepsilon}\right)\right.$ tends to $|M|(\Omega)$. The definition of $P_{\varepsilon}$ and formula (8.2) yield $P_{\varepsilon}(x)=x-\varepsilon v(x)$ for every $x \in \partial \Omega$, where $v$ is a smooth vectorfield. Hence the Lipschitz constant of the restriction of $P_{\varepsilon}$ to $\partial \Omega$ is of order $1+O(\varepsilon)$ and therefore $\left\|P_{\varepsilon \sharp}(M L \partial \Omega)\right\|$ tends to $|M|(\partial \Omega)$.

Combining these remarks and (8.5) we finally obtain

$$
\lim _{\varepsilon \rightarrow 0}\left\|M_{\varepsilon}\right\|=\lim _{\varepsilon \rightarrow 0}\left[\| M\left\llcorner\left(\Omega \backslash U_{\varepsilon}\right)\|+\| P_{\varepsilon \sharp}(M\llcorner\partial \Omega) \|]=|M|(\bar{\Omega})=\|M\| .\right.\right.
$$

The rest of the statement follows by standard arguments like those used in the proof of Proposition 8.6, and we omit the details. 


\section{Appendix B: Maps With Prescribed Jacobians}

The main result of this appendix is Theorem 9.6: it is a refinement of Theorem 5.10 of [3] and allows us to construct $S^{k-1}$-valued maps with prescribed Jacobian and prescribed boundary values. The proof requires some preliminary lemmas and additional notation.

We adopt the notation introduced in Section 4.1. In particular, given an $h$-dimensional polyhedral current, we denote by the same letter the supporting polyhedral set; given a simplex $F$ in $\mathbb{R}^{n+k}$ and $\delta, \gamma>0$, the set $U(F, \delta, \gamma)$ is the set of all $x \in \mathbb{R}^{n+k}$ that satisfy (4.1) or equivalently (4.2). Jacobians of maps of class $W^{1, k-1}$ valued in $S^{k-1}$ are intended in the distributional sense (see Remark 2.11).

9.1. Additional notation. For every integer $\sigma$ we fix a smooth map $\varphi_{\sigma}$ : $S^{k-1} \rightarrow S^{k-1}$ so that $\varphi_{0}$ is constant, $\varphi_{1}$ is the identity, and each $\varphi_{\sigma}$ has degree $\sigma$.

Let $F$ and $U=U(F, \delta, \gamma)$ be taken as above, and let $u: U \rightarrow S^{k-1}$ be a map which is continuous on $U \backslash F$. The degree of the singularity of $u$ at $F$ is $\sigma:=\operatorname{deg}\left(u, S, S^{k-1}\right)$, where $S$ is any $(k-1)$-dimensional sphere of the form $\left\{x^{\prime}\right\} \times r S^{k-1}$ contained in $U$. Note that $\sigma$ does not depend on the choice of $S$.

Let $M$ and $S$ be finite unions of simplexes in $\mathbb{R}^{n+k}$ with dimension at most $n$ and $n-1$, respectively. We say that a map $u$ on $\bar{\Omega}$ has a nice singularity at $M, S$, if $u$ is locally Lipschitz on $\bar{\Omega} \backslash(M \cup S)$ and

$$
|D u(x)|=O\left(\frac{1}{\operatorname{dist}(x, M)}\right)+O\left(\frac{1}{(\operatorname{dist}(x, S))^{p}}\right) \quad \text { for every } p>1 .
$$

We say that a map $u$ has a nice singularity at $M$ (resp., at $S$ ) if it is locally Lipschitz on $\bar{\Omega} \backslash M$ (resp., $\bar{\Omega} \backslash S$ ) and satisfies (9.1) when the second term (resp., the first term) at the right-hand side is dropped.

Remark 9.2. (i) Let $U^{\prime}$ be the interior of $U=U(F, \delta, \gamma)$. The Jacobian of a map $u \in W^{1, k-1}\left(U^{\prime}, S^{k-1}\right)$ which is continuous in $U^{\prime} \backslash F$ is supported on the singularity $F$, and more precisely $\star J u=\alpha_{k} \sigma F$, where $\sigma$ is the degree of the singularity-see $\left[3\right.$, Section 3.7]. ${ }^{23}$

(ii) If $u: \bar{\Omega} \rightarrow S^{k-1}$ has a nice singularity at $M, S$, then (9.1) and Lemma 8.3 imply $u \in W^{1, k-1}(\Omega \backslash(M \cup S))$, and therefore $u \in W^{1, k-1}(\Omega)$, because $M \cup S$ has codimension larger than $k-1$.

Lemma 9.3. Let $F$ be an $n$-dimensional simplex in $\mathbb{R}^{n+k}$, take $U=U(F, \delta, \gamma)$ as above, and let $U^{\prime}$ denote the interior of $U$. Given a map $u: U \rightarrow S^{k-1}$ with a nice singularity at $F$ of degree $\sigma$, there exists $u^{\prime}: U \rightarrow S^{k-1}$ such that

\footnotetext{
${ }^{23}$ We assume that the orientation of $F$ agrees with that of $\mathbb{R}^{n}$, and that the orientation of $\mathbb{R}^{n} \times \mathbb{R}^{k}$ agrees with that of $\mathbb{R}^{n+k}$.
} 
(i) $u^{\prime}=u$ on $\partial U$;

(ii) $u^{\prime}$ has a nice singularity at $F, \partial F$ of degree $\sigma$;

(iii) $u^{\prime} \in W^{1, k-1}\left(U^{\prime}, S^{k-1}\right)$ and $J u^{\prime}=J u$;

(iv) $u^{\prime}(x)=\varphi_{\sigma}\left(x^{\prime \prime} /\left|x^{\prime \prime}\right|\right)$ for all $x \in U(F, \delta / 4, \gamma / 4)$.

Proof. Throughout this proof, the letter $C$ denotes any positive finite constant, possibly different at each occurrence, that depends only on the choice of $u$, $U$, and of the map $\Psi$ below.

We define $g$ as in (4.2), and then $U$ can be written as $U_{1} \cup U_{2}$, where $U_{1}$ is the set of all $x$ such that $x^{\prime} \in F$ and $g\left(x^{\prime}\right) / 2 \leq\left|x^{\prime \prime}\right| \leq g\left(x^{\prime}\right)$, while $U_{2}$ is the set of all $x$ such that $x^{\prime} \in F$ and $\left|x^{\prime \prime}\right| \leq g\left(x^{\prime}\right) / 2$ (see Figure 9.1).
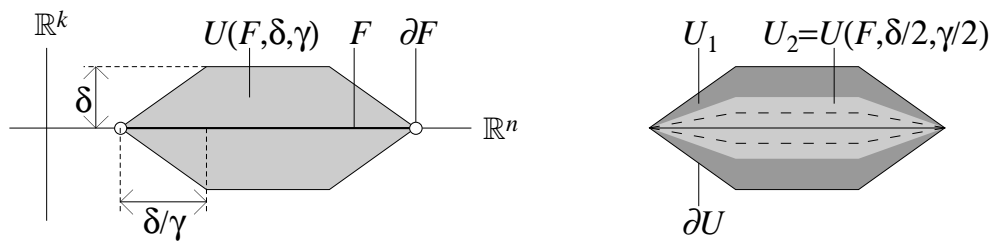

FIGURE 9.1.

Thus $U_{2}=U(F, \delta / 2, \gamma / 2), \partial U_{1}=\partial U \cup \partial U_{2}$, and for every $x \in U_{1}$

$$
\left|x^{\prime \prime}\right| \leq g\left(x^{\prime}\right) \leq \gamma \operatorname{dist}\left(x^{\prime}, \partial F\right) \leq \gamma \operatorname{dist}(x, \partial F) \leq C\left|x^{\prime \prime}\right| .
$$

In other words, the ratio between any two of the quantities $\left|x^{\prime \prime}\right|, g\left(x^{\prime}\right)$, dist $\left(x^{\prime}, \partial F\right)$, and $\operatorname{dist}(x, \partial F)$ is bounded over all $x \in U_{1}$.

The proof is divided in two steps. First we define $u^{\prime}$ in $U_{1}$ so that it agrees with $u$ on $\partial U$, and depends only on the variable $x^{\prime \prime} /\left|x^{\prime \prime}\right|$ on $\partial U_{2}$. Then we extend $u^{\prime}$ to the interior of $U_{2}$ so that $u^{\prime}(x)=\varphi_{\sigma}\left(x^{\prime \prime} /\left|x^{\prime \prime}\right|\right)$ for $x \in U(F, \delta / 4, \gamma / 4)$. From the topological point of view, the first step is almost immediate, because $U_{1} \backslash \partial F$ is homeomorphic to the product $(F \backslash \partial F) \times S^{k-1} \times\left[\frac{1}{2}, 1\right]$ and $F \backslash \partial F$ is contractible; the required estimate on $\left|D u^{\prime}\right|$, however, is delicate. The second step is taken from Section 5.9 of [3], and will not be explained in full detail.

Step 1. Since $F$ is convex, there exists a Lipschitz retraction $\Psi: F \times[0,1] \rightarrow F$ such that $\Psi\left(x^{\prime}, 0\right)=x^{\prime}$ and $\Psi\left(x^{\prime}, 1\right)=x_{0}^{\prime}$ for every $x^{\prime} \in F$, where $x_{0}^{\prime}$ is an arbitrary point in the interior of $F$; we can also assume that

$$
\operatorname{dist}\left(\Psi\left(x^{\prime}, t\right), \partial F\right) \geq \operatorname{dist}\left(x^{\prime}, \partial F\right)+C t
$$

for every $x^{\prime} \in F, t \in[0,1]$ (take for example $\left.\Psi\left(x^{\prime}, \tau\right):=(1-\tau) x^{\prime}+\tau x_{0}^{\prime}\right)$.

We choose a decreasing function $\tau:\left[\frac{1}{2}, 1\right] \rightarrow[0,1]$ of class $C^{1}$ such that $\tau(1)=0$ and $\tau\left(\frac{1}{2}\right)=1$ (to be properly chosen later), and for every $x \in U_{1} \backslash \partial F$ 
we set

$$
\Psi^{\prime}(x):=\Psi\left(x^{\prime}, \tau\left(\frac{\left|x^{\prime \prime}\right|}{g\left(x^{\prime}\right)}\right)\right), \quad u^{\prime}(x):=u\left(\Psi^{\prime}(x), \frac{g\left(\Psi^{\prime}(x)\right)}{g\left(x^{\prime}\right)} x^{\prime \prime}\right) .
$$

One easily checks that $u^{\prime}$ is locally Lipschitz on $U_{1} \backslash \partial F$, it agrees with $u$ on $\partial U-$ and therefore statement (i) holds-and for every $x \in \partial U_{2}$ it can be written as $u^{\prime}(x)=\varphi\left(x^{\prime \prime} /\left|x^{\prime \prime}\right|\right)$, with $\varphi: S^{k-1} \rightarrow S^{k-1}$ Lipschitz.

Now we estimate $\left|D u^{\prime}\right|$. Taking into account (9.2) and the fact that $\Psi$ and $g$ are Lipschitz, from (9.4) we obtain

$$
\begin{aligned}
\left|D \Psi^{\prime}(x)\right| & \leq C|D \Psi|\left[1+|\dot{\tau}|\left(\frac{\left|D x^{\prime \prime}\right|}{g\left(x^{\prime}\right)}+\frac{\left|D g\left(x^{\prime}\right)\right|\left|x^{\prime \prime}\right|}{g^{2}\left(x^{\prime}\right)}\right)\right] \\
& \leq C\left[1+\frac{|\dot{\tau}|}{\left|x^{\prime \prime}\right|}\right],
\end{aligned}
$$

where the argument of $\tau$ and $\dot{\tau}$ is $\left|x^{\prime \prime}\right| / g\left(x^{\prime}\right)$, and the argument of $\Psi$ is $\left(x^{\prime}, \tau\right)$. From (9.4) and (9.5) we obtain ${ }^{24}$

$$
\begin{aligned}
\left|D u^{\prime}(x)\right| & \leq C|D u|\left[\left|D \Psi^{\prime}\right|+\frac{\left|D \Psi^{\prime}\right|\left|x^{\prime \prime}\right|+g\left(\Psi^{\prime}\right)}{g\left(x^{\prime}\right)}+\frac{g\left(\Psi^{\prime}\right)\left|x^{\prime \prime}\right|}{g^{2}\left(x^{\prime}\right)}\right] \\
& \leq C \frac{g\left(x^{\prime}\right)}{g\left(\Psi^{\prime}\right)\left|x^{\prime \prime}\right|}\left[1+\frac{|\dot{\tau}|}{\left|x^{\prime \prime}\right|}+\frac{g\left(\Psi^{\prime}\right)}{g\left(x^{\prime}\right)}\right] \leq \frac{C}{\left|x^{\prime \prime}\right|}\left[1+\frac{|\dot{\tau}|}{g\left(\Psi^{\prime}\right)}\right] \\
& \leq \frac{C}{\operatorname{dist}(x, \partial F)}\left[1+\frac{|\dot{\tau}|}{\operatorname{dist}(x, \partial F)+\tau}\right] .
\end{aligned}
$$

Let $f:[0,+\infty) \rightarrow[0,+\infty)$ be a continuous and strictly increasing function-to be properly chosen below—such that $f(0)=0, f(t) / t$ is decreasing in $\left(0, t_{0}\right]$ for some $t_{0}>0$ and

$$
\int_{0}^{t_{0}} \frac{d t}{f(t)}<\frac{1}{2}
$$

By (9.7) we can find a decreasing function $\tau:\left[\frac{1}{2}, 1\right] \rightarrow[0,1]$ of class $C^{1}$ such that $\tau\left(\frac{1}{2}\right)=1, \tau(1)=0$, and $\dot{\tau}=-f(\tau)$ in the interval of all points where $\tau \leq t_{0}$. For every $d>0$ and every point where $\tau \leq t_{0}$ there holds

$$
\frac{|\dot{\tau}|}{d+\tau}=\frac{f(\tau)}{d+\tau} \leq \frac{f(d)}{d}
$$

\footnotetext{
${ }^{24}$ The argument of $D u$ in the first line is the same as that of $u$ in formula (9.4). The second inequality follows by estimates (9.2), (9.5) and $|D u(x)| \leq C /\left|x^{\prime \prime}\right|-$ by assumption, $u$ has a nice singularity at $F$. For the third inequality we used the estimate $g\left(\Psi^{\prime}\right)=g\left(\Psi\left(x^{\prime}, \tau\right)\right) \geq g\left(x^{\prime}\right)$, while the last one follows from (9.2) and $g\left(\Psi^{\prime}\right) \geq C$ (dist $\left.\left(x^{\prime}, \partial F\right)+\tau\right)$. Both estimates follow from (9.3).
} 
(if $d \leq \tau$ use that $f(t) / t$ is decreasing, and if $d \geq \tau$ use that $f(t)$ is increasing). Therefore estimate (9.6) becomes

$$
\left|D u^{\prime}(x)\right| \leq C \frac{f(\operatorname{dist}(x, \partial F))}{(\operatorname{dist}(x, \partial F))^{2}} \quad \text { for every } x \in U_{1} .
$$

Step 2. To extend $u^{\prime}$ to $U_{2}$, we proceed as in Section 5.9 of [3]. Since both $\varphi$ and $\varphi_{\sigma}$ have degree $\sigma$, by the Hopf theorem they are homotopic, and we can construct a Lipschitz map $\Phi: S^{k-1} \times\left[0, \frac{1}{2}\right]$ such that $\Phi\left(\cdot, \frac{1}{2}\right)=\varphi(\cdot)$ and $\Phi(\cdot, t)=\varphi_{\sigma}(\cdot)$ for $t \leq \frac{1}{4}$. For every $x$ in the interior of $U_{2}$ we set

$$
u^{\prime}(x):=\Phi\left(\frac{x^{\prime \prime}}{\left|x^{\prime \prime}\right|}, \frac{\left|x^{\prime \prime}\right|}{g\left(x^{\prime}\right)}\right) .
$$

It is clear that $u^{\prime}$ is locally Lipschitz on $U \backslash F$, satisfies statement (iv), and has a singularity of degree $\sigma$ at $F$. A straightforward computation gives $\left|D u^{\prime}(x)\right|=$ $O\left(1 /\left|x^{\prime \prime}\right|\right)$ for $x \in U_{2}$. Together with (9.8), this implies

$$
\left|D u^{\prime}(x)\right|=O\left(\frac{1}{\operatorname{dist}(x, F)}\right)+O\left(\frac{f(\operatorname{dist}(x, \partial F))}{(\operatorname{dist}(x, \partial F))^{2}}\right) .
$$

In particular, taking $f(t):=t(\log t)^{2}$ (for $t \leq 1 / e^{2}$ ), we obtain that $u^{\prime}$ has a nice singularity at $F, \partial F$, and the proof of statement (ii) is complete. In turn, statement (ii) implies that $u^{\prime}$ belongs to $W^{1, k-1}\left(U^{\prime}, S^{k-1}\right)$ (cf. Remark 9.2(ii)), and $\star J u=\star J u^{\prime}$ because the singularities of $u$ and $u^{\prime}$ have both degree $\sigma$ (cf. Remark 9.2(i)). This concludes the proof of statement (iii).

Remark 9.4. (i) If $u$ has a singularity of degree $\sigma=0$ at $F$, then definition (9.9) and the fact that $\varphi_{0}$ is a constant map imply that the map $u^{\prime}$ in Lemma 9.3 is constant in $U_{2}$. Thus the first term at the right-hand side of estimate (9.10) can be dropped, and $u^{\prime}$ has a nice singularity at $\partial F$. In other words, Lemma 9.3 shows that $n$-dimensional singularities of degree 0 can be reduced to $(n-1)$-dimensional singularities.

(ii) The function $f(t)$ in the proof of Lemma 9.3 cannot be taken asymptotically equivalent to $t$ because of assumption (9.7), and therefore our construction does not provide a map $u^{\prime}$ such that $\left|D u^{\prime}(x)\right|=O(1 / \operatorname{dist}(x, \partial F))$.

In the following we assume that $\Omega$ is a bounded domain in $\mathbb{R}^{n+k}$ with polyhedral boundary, and $u: \bar{\Omega} \rightarrow \mathbb{R}^{k}$ a piecewise affine map. By Proposition 6.4(ii), we can choose a regular value $a \in B_{1}^{k}$ of $u$ such that the map $u_{a}$ defined in (6.7) belongs to $W^{1, k-1}\left(\Omega, S^{k-1}\right)$, and $\star J u_{a}=\alpha_{k} M_{a}(u)$. We also choose a regular value $y \in S^{k-1}$ of $u_{a}$ such that $M_{a}(u)=(-1)^{n-1} \partial M_{y}\left(u_{a}\right)$ in $\Omega$-see Proposition 5.3(iii) and Remark 5.4(i). Note that $M_{a}(u)$ and $M_{y}\left(u_{a}\right)$ are polyhedral current of dimension $n$ and $n+1$, supported on the sets $u^{-1}(a)$ and $u^{-1}\left(L_{a, y}\right)$, where $L_{a, y}$ is the half-line $\{a+t y: t \geq 0\}$. 
Lemma 9.5. Let $\Omega, u, a$, and $y$ be taken as above. Let $\Omega_{1}$ be an open set relatively compact in $\Omega$ whose boundary is polyhedral and transversal to $M_{a}(u)$ and $M_{y}\left(u_{a}\right)$ (in the sense of Note (16)) and set $\Omega_{2}:=\Omega \backslash \bar{\Omega}_{1}$. Let $N^{\prime}:=M_{y}\left(u_{a}\right)\left\llcorner\Omega_{2}\right.$ be the restriction of the polyhedral current $M_{y}\left(u_{a}\right)$ to $\Omega_{2}$, and let $M^{\prime}$ be the boundary of $N^{\prime}$ in $\Omega$. Given $y^{\prime} \in S^{k-1}$ with $y^{\prime} \neq y$, there exists a map $u^{\prime}: \bar{\Omega}_{2} \rightarrow S^{k-1}$ such that

(i) $u^{\prime}=u_{a}$ on $\partial \Omega$ and $u^{\prime}=y^{\prime}$ on $\partial \Omega_{1}$;

(ii) $u^{\prime}$ has a nice singularity at $M^{\prime}$;

(iii) $u^{\prime} \in W^{1, k-1}\left(\Omega_{2}, S^{k-1}\right)$ and $M_{y}\left(u^{\prime}\right)=N^{\prime}$.

Proof. Throughout this proof, the letter $C$ denotes any positive finite constant, possibly different at each occurrence, that depends only on the choice of $\Omega$, $\Omega_{1}, u, a, y$, and $y^{\prime}$.

First of all, we choose a map $\Psi: S^{k-1} \times[0,+\infty) \rightarrow S^{k-1}$ such that

(i') $\Psi(x, t)=x$ if $t \geq 1$;

(ii') $\Psi(x, t)=y^{\prime}$ if $|x-y| \geq K t$;

(iii') $\Psi$ is of class $C^{1}$ on the complement of $(y, 0)$, and $|D \Psi(x, t)| \leq C / t$.

(iv') for $t>0, \Psi(x, t)=y$ if and only if $x=y$, and the derivative of $\Psi(\cdot, t)$ at $y$ is orientation preserving.

(A way to construct the map $\Psi$ is the following: represent $S^{k-1}$ as a quotient of the closed unit ball $D$ in $\mathbb{R}^{k-1}$ so that 0 corresponds to $y$ and $\partial D$ corresponds to $y^{\prime}$, and take $\Psi(x, t):=x /(t \wedge 1)$ for $|x| \leq t$ and $\Psi(x, t):=x /|x| \simeq y^{\prime}$ for $|x| \geq t$.)

We set $M^{\prime \prime}:=M_{a}(u) \cap \bar{\Omega}_{2}$, thus $M^{\prime \prime}$ is the closure of $M^{\prime} \cap \Omega_{2}\left(\right.$ or $\left.\partial N^{\prime} \cap \Omega_{2}\right)$. Then, for every $x \in \bar{\Omega}_{2}$, we set

$$
u^{\prime}(x):=\Psi\left(u_{a}(x), \sigma(x) g_{1}(x)\right), \quad \text { where } g_{1}(x):=\frac{\operatorname{dist}\left(x, M^{\prime}\right)}{\operatorname{dist}\left(x, M^{\prime \prime}\right) \wedge 1},
$$

where $\sigma$ is a positive Lipschitz function on $\Omega_{2}$ bounded away from 0 that will be made precise in the following. The verification of the properties of $u^{\prime}$ is divided in several steps.

Step 1. Formula (9.11) and assumption (iii') imply that $u^{\prime}$ is well-defined and locally Lipschitz in the set of all $x \in \Omega_{2}$ such that $\left(u_{a}(x), \sigma(x) g_{1}(x)\right) \neq(y, 0)$ and $\operatorname{dist}\left(x, M^{\prime \prime}\right)>0$, that is, in the complement of the set $M^{\prime}$.

Step 2. We estimate now $\left|D u^{\prime}\right|$. The definition of $g_{1}$ yields

$$
\begin{aligned}
\left|D g_{1}(x)\right| & \leq \frac{1}{\operatorname{dist}\left(x, M^{\prime \prime}\right) \wedge 1}+\frac{\operatorname{dist}\left(x, M^{\prime}\right)}{\left(\operatorname{dist}\left(x, M^{\prime \prime}\right) \wedge 1\right)^{2}} \\
& \leq \frac{C}{\operatorname{dist}\left(x, M^{\prime \prime}\right)} .
\end{aligned}
$$


Since $u$ is piecewise affine, $u_{a}$ has a nice singularity at $M_{a}(u)$, that is, $\left|D u_{a}(x)\right| \leq$ $C / \operatorname{dist}\left(x, M^{\prime \prime}\right)$ for every $x \in \Omega_{2}$. Hence, recalling the estimate on $|D \Psi|$ in assumption (iii') and (9.12), we obtain

$$
\begin{aligned}
\left|D u^{\prime}(x)\right| & \leq|D \Psi| \cdot\left(\left|D u_{a}\right|+|D \sigma| g_{1}+\sigma\left|D g_{1}\right|\right) \\
& \leq \frac{C}{\sigma g_{1}} \cdot \frac{C}{\operatorname{dist}\left(x, M^{\prime \prime}\right)} \leq \frac{C}{\operatorname{dist}\left(x, M^{\prime}\right)} .
\end{aligned}
$$

Together with Step 1, this proves statement (ii).

Step 3. An easy computation shows that $g_{1}(x) \geq 1 \wedge \operatorname{dist}\left(\partial \Omega, \partial \Omega_{1}\right)$ for every $x \in \partial \Omega$, and therefore assumption (i') and formula (9.11) imply $u^{\prime}(x)=u_{a}(x)$ for every $x \in \partial \Omega$ provided that $\sigma \geq 1 /\left(1 \wedge \operatorname{dist}\left(\partial \Omega, \partial \Omega_{1}\right)\right)$ on $\partial \Omega$.

Step 4. Using the definitions of $u_{a}$ and $N^{\prime}$, and the fact that $u$ is piecewise affine, it is not difficult to check that for every $x \in \bar{\Omega}$ there holds ${ }^{25}$

$$
\left|u_{a}(x)-y\right| \geq C \frac{\operatorname{dist}\left(x, M_{y}\left(u_{a}\right)\right)}{\operatorname{dist}\left(x, M_{a}(u)\right)} \vee C^{\prime} \operatorname{dist}\left(x, M_{y}\left(u_{a}\right)\right) .
$$

Thus, for every $x \in \Omega_{2}$,

$$
\left|u_{a}(x)-y\right| \geq C g_{2}(x), \quad \text { where } g_{2}(x):=\frac{\operatorname{dist}\left(x, N^{\prime}\right)}{\operatorname{dist}\left(x, M^{\prime \prime}\right) \wedge 1} .
$$

By assumption (ii'), $u^{\prime}(x)=y^{\prime}$ provided that $\left|u_{a}(x)-y\right| \geq K \sigma(x) g_{1}(x)$. Taking into account (9.13) and the definitions of $g_{1}$ and $g_{2}$, we obtain the implication

$$
\sigma(x) \operatorname{dist}\left(x, M^{\prime}\right) \geq C \operatorname{dist}\left(x, N^{\prime}\right) \Rightarrow u^{\prime}(x)=y^{\prime} .
$$

On the other hand, $N^{\prime}$ is transversal to $\partial \Omega_{1}$, and then

$$
C \operatorname{dist}\left(x, N^{\prime}\right) \geq \operatorname{dist}\left(x, N^{\prime} \cap \partial \Omega_{1}\right),
$$

for every $x \in \partial \Omega_{1}$ (cf. Note (16)). Since $N^{\prime} \cap \partial \Omega_{1} \subset M^{\prime}$,

$$
C \operatorname{dist}\left(x, N^{\prime}\right) \geq \operatorname{dist}\left(x, M^{\prime}\right) \quad \text { for every } x \in \partial \Omega_{1} .
$$

Putting together (9.14) and (9.15) we finally obtain that $u^{\prime}(x)=y^{\prime}$ on $\partial \Omega_{1}$ provided that $\sigma$ is smaller than a given positive constant on $\partial \Omega_{1}$. Together with Step 3, this proves statement (i).

\footnotetext{
${ }^{25}$ This estimate holds provided that $M_{a}(u)$ and $M_{y}\left(u_{a}\right)$ are not empty. It follows by the fact that the quantities in the left- and in the right-hand side vanish on the same set $M_{y}\left(u_{a}\right)$, and that $u$ is locally affine and non-degenerate in the directions orthogonal to $M_{a}(u)$.
} 
Step 5. $u^{\prime}$ belongs to $W^{1, k-1}\left(\Omega, S^{k-1}\right)$ because it has a nice singularity at $M^{\prime}$ (cf. Remark 9.2(ii)). By assumption (iv'), $u^{\prime}(x)=y$ if and only if $u_{a}(x)=$ $y$, and since $\Psi(\cdot, t)$ is orientation-preserving at $y$, we deduce that $M_{y}\left(u^{\prime}\right)=$ $M_{y}\left(u_{a}\right)\left\llcorner\Omega_{2}=N^{\prime}\right.$, and the proof of statement (iii) is concluded.

We can now state and prove the main result of this section.

Theorem 9.6. Let $\Omega, u, a$, and $y$ be taken as above, and let $M$ be an $n$ dimensional polyhedral current of the form $M=M_{a}(u)+\partial N$, where $N$ is a polyhedral current with compact support in $\Omega$. Then there exists a map $\bar{u}: \bar{\Omega} \rightarrow S^{k-1}$, an $(n-1)$-dimensional polyhedral set $S$, and $\delta, \gamma>0$ such that

(i) $\tilde{u}=u_{a}$ on $\partial \Omega$;

(ii) $\tilde{u}$ has a nice singularity at $M, S$;

(iii) $\tilde{u} \in W^{1, k-1}\left(\Omega, S^{k-1}\right)$ and $\star J \tilde{u}=\alpha_{k} M$;

(iv) for every $n$-dimensional face $F$ of $M$ there holds $u(x)=\varphi_{\sigma}\left(x^{\prime \prime} /\left|x^{\prime \prime}\right|\right)$ on $U(F, \delta, \gamma)$, where $\sigma$ is the multiplicity of $M$ on $F$.

Proof. We choose a point $y^{\prime} \neq y$ in $S^{k-1}$ and a polyhedral open set $\Omega_{1}$ such that $\operatorname{supp}(N) \Subset \Omega_{1} \Subset \Omega$ and $\partial \Omega_{1}$ is transversal to $M_{a}(u)$ and $M_{y}\left(u_{a}\right)$ (in the sense of Note (16)). Then we take $u^{\prime}$ as in Lemma 9.5.

We denote by $N^{\prime \prime}$ the restriction of $M_{y}\left(u_{a}\right)+(-1)^{n-1} N$ to $\Omega_{1}$, and by $M^{\prime \prime}$ the union of the $n$-dimensional faces of $N^{\prime \prime}$. We take $\delta, \gamma>0$ so that $U(F, \delta, \gamma)$ is contained in the closure of $\Omega_{1}$ for every $(n+1)$-dimensional face $F$ of $N^{\prime \prime}$. By Proposition 5.8 in [3] we can find a map $u^{\prime \prime}: \mathbb{R}^{n+k} \rightarrow S^{k-1}$ such that

(i') $u^{\prime \prime}=y^{\prime}$ in $\mathbb{R}^{n+k} \backslash \cup U(F, \delta, \gamma)$, where the union is taken over all $(n+1)$ dimensional faces $F$ of $N^{\prime \prime}$; in particular $u^{\prime \prime}=y^{\prime}$ in $\mathbb{R}^{n+k} \backslash \Omega_{1}$;

(ii') $u^{\prime \prime}$ is locally Lipschitz in $\mathbb{R}^{n+k} \backslash M^{\prime \prime}$, and $\left|D u^{\prime \prime}(x)\right|=O\left(1 / \operatorname{dist}\left(x, M^{\prime \prime}\right)\right)$; (iii') $u^{\prime \prime} \in W_{\text {loc }}^{1, k-1}\left(\mathbb{R}^{n+k}, S^{k-1}\right)$ and $\star J u^{\prime \prime}=\alpha_{k} \partial N^{\prime \prime}$.

We set

$$
\tilde{u}(x):= \begin{cases}u^{\prime}(x) & \text { for } x \in \bar{\Omega} \backslash \Omega_{1}, \\ u^{\prime \prime}(x) & \text { for } x \in \Omega_{1} .\end{cases}
$$

Taking into account the properties of $u^{\prime \prime}$ and $u^{\prime}$ (see Lemma 9.5), and in particular that $u^{\prime \prime}=u^{\prime}$ on $\partial \Omega_{1}$, one easily verifies that $\tilde{u}$ agrees with $u_{a}$ on $\partial \Omega$, is locally Lipschitz in the complement of $M^{\prime} \cup M^{\prime \prime}$, satisfies

$$
|D \tilde{u}(x)|=O\left(\frac{1}{\operatorname{dist}\left(x, M^{\prime} \cup M^{\prime \prime}\right)}\right),
$$

and belongs to $W^{1, k-1}\left(\Omega, S^{k-1}\right)$. Moreover, $M_{y}(\tilde{u})=M_{y}\left(u_{a}\right)+(-1)^{n-1} N$ by construction, and then

$$
\star J \tilde{u}=(-1)^{n-1} \alpha_{k} \partial M_{y}(\tilde{u})=\alpha_{k} M .
$$

Thus $\tilde{u}$ satisfies statements (i) and (iii), and has a nice singularity at $M^{\prime} \cup M^{\prime \prime}$. 
Now we use Lemma 9.4 to modify $\tilde{u}$ in each $U(F, \delta, \gamma)$ so that it agrees with $\varphi_{\sigma}$ in $U(F, \delta / 4, \gamma / 4)$, where $\sigma$ is the degree of the singularity of $\tilde{u}$ at $F$. Hence $\tilde{u}$ satisfies statement (iv).

By Remark 9.4(ii) we have that $\tilde{u}$ is no longer singular at $F$ if $\sigma=0$, and since $\sigma$ agrees with the multiplicity of $M$ at $F$ (Remark 9.2(i)), $\sigma=0$ if and only if $F$ does not belong to the support of $M$. Hence $\tilde{u}$ has a nice singularity at $M, S$, where $S$ is the union of the $(n-1)$-dimensional faces of $M^{\prime} \cup M^{\prime \prime}$.

Remark 9.7. By statement (i) of Theorem 9.6, the map $\tilde{u}$ satisfies estimate (9.1) for every $p>1$. It seems possible to choose $\tilde{u}$ so that (9.1) holds with $p=1$, that is, $|D \tilde{u}(x)|=O(1 / \operatorname{dist}(x, M \cup S))$. However, the only proof we could find is completely different from the current one and more complicated. Hence we opted for a slightly weaker statement, which is anyhow more than enough for our purposes.

Acknowledgements. This research was supported by italian CNR through grant 99.01699.CT01, and by MURST research project "Calcolo delle Variazioni." The third author was supported by CNR also through grants 203.01.69 and 203.01.71, while visiting ENS Cachan and the University of Paris VI; he thanks these institutions for the hospitality. During the final stage of this research, the first author was visiting the University of Paris VI and the Max Planck Institute for Mathematics in the Sciences in Leipzig, whose support and hospitality he gratefully acknowledges. Finally, we would like to thank one of the referees for his careful check of the manuscript, and Niccolò Desenzani for pointing out a serious mistake in Section 7.

\section{REFERENCES}

[1] Giovanni Alberti, Variational models for phase transitions, an approach via $\Gamma$-convergence, Calculus of Variations and Partial Differential Equations (Pisa, 1996) (G. . Butazzo et al., eds.), Springer, Berlin, 2000, pp. 95-114. MR1757697

[2] _ـ Un risultato di convergenza variazionale per funzionali di tipo Ginzburg-Landau in dimensiona qualunque (A variational convergence result for Ginzburg-Landau functionals in any dimension), Boll. Unione Mat. Ital. Sez. B Artic. Ric. Mat. (8) 4 (2001), 289-310. MR1831990 (2002g:35053) (Italian)

[3] Giovanni Alberti, Sisto Baldo, and Giandomenico Orlandi, Functions with prescribed singularities, J. Eur. Math. Soc. (JEMS) 5 (2003), 275-311, http://dx.doi.org/10.1007/s10097-003-0053-5. MR2002215 (2004g:49068)

[4] Giovanni Alberti, Guy Bouchitté, and Pierre Seppecher, Un résultat de perturbations singulières avec la norme $H^{1 / 2}$, C. R. Acad. Sci. Paris Sér. I Math. 319 (1994), 333-338. MR1289307 (95c:49015) (French with long English summary)

[5] SISTO BALDO, Minimal interface criterion for phase transitions in mixtures of Cahn-Hilliard fluids, Ann. Inst. H. Poincaré Anal. Non Linéaire 7 (1990), 67-90. MR1051228 (91j:76005) (English, with French summary) 
[6] Sisto Baldo and Giandomenico Orlandi, Codimension one minimal cycles with coefficients in $\mathbb{Z}$ or $\mathbb{Z} p$, and variational functionals on fibered spaces, J. Geom. Anal. 9 (1999), 547-568. MR1757578 (2001c:49071)

[7] _ Fiber bundles and regular approximation of codimension-one cycles, Ann. Global Anal. Geom. 20 (2001), 47-57, http://dx.doi.org/10.1023/A:1010682522618. MR1846896 (2002g:49073)

[8] Fabrice Bethuel, Jean Bourgain, Haïm Brezis, and Giandomenico Orlandi, $W^{1, p}$ estimates for solutions to the Ginzburg-Landau equation with boundary data in $H^{1 / 2}$, C. R. Acad. Sci. Paris Sér. I Math. 333 (2001), 1069-1076. MR1881236 (2003d:35078) (English, with English and French summaries)

[9] Fabrice Bethuel, Haïm Brezis, and Frédéric Hélein, Ginzburg-Landau Vortices, Progress in Nonlinear Differential Equations and their Applications, 13, Birkhäuser Boston Inc., Boston, MA, 1994, ISBN 0-8176-3723-0. MR1269538 (95c:58044)

[10] _ Asymptotics for the minimization of a Ginzburg-Landau functional, Calc. Var. Partial Differential Equations 1 (1993), 123-148, http://dx.doi.org/10.1007/BF01191614. MR1261720 (94m:35083)

[11] FABRice Bethuel and Tristan Rivière, Vortices for a variational problem related to superconductivity, Ann. Inst. H. Poincaré Anal. Non Linéaire 12 (1995), 243-303. MR1340265 (96g:35045) (English, with English and French summaries)

[12] RaOul BotT and Loring W. Tu, Differential Forms in Algebraic Topology, Graduate Texts in Mathematics, vol. 82, Springer-Verlag, New York, 1982, ISBN 0-387-90613-4. MR658304 (83i:57016)

[13] Jean Bourgain, Haïm Brezis, and Petru Mironescu, On the structure of the Sobolev space $H^{1 / 2}$ with values into the circle, C. R. Acad. Sci. Paris Sér. I Math. 331 (2000), 119-124. MR1781527 (2001m:46068) (English, with English and French summaries)

[14] Andrea Braides, $\Gamma$-convergence for Beginners, Oxford Lecture Series in Mathematics and its Applications, vol. 22, Oxford University Press, Oxford, 2002, ISBN 0-19-850784-4. MR1968440 (2004e:49001)

[15] Haïm Brezis, Jean-Michel Coron, and Elliott H. Lieb, Harmonic maps with defects, Comm. Math. Phys. 107 (1986), 649-705, http://dx.doi.org/10.1007/BF01205490. MR868739 (88e:58023)

[16] HaÏm BREZIS and Petru Mironescu, Sur une conjecture de E. De Giorgi relative à l'énergie de Ginzburg-Landau, C. R. Acad. Sci. Paris Sér. I Math. 319 (1994), 167-170. MR1288397 (95h:49016) (French)

[17] Gianni Dal Maso, An Introduction to $\Gamma$-convergence, Progress in Nonlinear Differential Equations and their Applications, 8, Birkhäuser Boston Inc., Boston, MA, 1993, ISBN 0-8176-3679X. MR1201152 (94a:49001)

[18] Ennio De Giorgi and Tullio Franzoni, Su un tipo di convergenza variazionale, Atti Accad. Naz. Lincei Rend. Cl. Sci. Fis. Mat. Natur. (8) 58 (1975), 842-850. MR0448194 (56 \#6503) (Italian)

[19] Niccolò DesenZani and Ilaria Fragalà, Concentration of Ginzburg-Landau energies with supercritical growth, SIAM J. Math. Anal. (to appear).

[20] Herbert Federer, Geometric Measure Theory, Die Grundlehren der mathematischen Wissenschaften, Band 153, Springer-Verlag New York Inc., New York, 1969. MR0257325 (41 \#1976) 
[21] Mariano Giaquinta, Giuseppe Modica, and JiŘí SouČEK, Cartesian Currents in the Calculus of Variations. I, Ergebnisse der Mathematik und ihrer Grenzgebiete. 3. Folge. A Series of Modern Surveys in Mathematics [Results in Mathematics and Related Areas. 3rd Series. A Series of Modern Surveys in Mathematics], vol. 37, Springer-Verlag, Berlin, 1998, ISBN 3-540-640096. MR1645086 (2000b:49001a)

[22] Zheng-ChaO HAN and YAN YAN LI, Degenerate elliptic systems and applications to Ginzburg-Landau type equations. I, Calc. Var. Partial Differential Equations 4 (1996), 171202, http://dx.doi.org/10.1007/s005260050034. MR1379199 (97d:35085a). Erratum: ibid., 4 (1996), 497.

[23] Fengbo Hang and Fang-Hua Lin, A remark on the Jacobians, Commun. Contemp. Math. 2 (2000), 35-46, http://dx.doi.org/10.1142/S0219199700000037. MR1753137 (2001f:49082)

[24] Robert Hardt and FANG-HUA Lin, Mappings minimizing the $L^{p}$ norm of the gradient, Comm. Pure Appl. Math. 40 (1987), 555-588. MR896767 (88k:58026)

[25] Min-Chun Hong, Asymptotic behavior for minimizers of a Ginzburg-Landau-type functional in higher dimensions associated with n-harmonic maps, Adv. Differential Equations 1 (1996), 611634. MR1401406 (97h:58047)

[26] Arthur Jaffe and Clifford Taubes, Vortices and Monopoles, Progress in Physics, vol. 2, Birkhäuser Boston, Mass., 1980, ISBN 3-7643-3025-2. MR614447 (82m:81051)

[27] Robert L. JERRARD, Lower bounds for generalized Ginzburg-Landau functionals, SIAM J. Math. Anal. 30 (1999), 721-746, http://dx.doi.org/10.1137/S0036141097300581. MR1684723 (2001f:35115)

[28] Robert L. Jerrard and Halil Mete Soner, Functions of bounded higher variation, Indiana Univ. Math. J. 51 (2002), 645-677, http://dx.doi.org/10.1512/iumj.2002.51.2229. MR1911049 (2003e:49069)

[29] _ The Jacobian and the Ginzburg-Landau energy, Calc. Var. Partial Differential Equations 14 (2002), 151-191, http://dx.doi.org/10.1007/s005260100093. MR1890398 (2003d:35069)

[30] FAng-Hua LiN and Tristan Rivière, Complex Ginzburg-Landau equations in high dimensions and codimension two area minimizing currents, J. Eur. Math. Soc. (JEMS) 1 (1999), 237-311. MR1714735 (2000g:49048). Erratum: ibid. 2 (2000), 87-91. MR1750451 (2001a:49041).

[31] LUCIANO MODICA, The gradient theory of phase transitions and the minimal interface criterion, Arch. Rational Mech. Anal. 98 (1987), 123-142, http://dx.doi.org/10.1007/BF00251230. MR866718 (88f:76038)

[32] Luciano Modica and Stefano Mortola, Un esempio di $\Gamma^{-}$-convergenza, Boll. Un. Mat. Ital. B (5) 14 (1977), 285-299. MR0445362 (56 \#3704) (Italian, with English summary)

[33] _ Il limite nella $\Gamma$-convergenza di una famiglia di funzionali ellittici, Boll. Un. Mat. Ital. A (5) 14 (1977), 526-529. MR0473971 (57 \#13628) (Italian, with English summary)

[34] J. Alberto Montero, Peter Sternberg, and William P. Ziemer, Local minimizers with vortices in the Ginzburg-Landau system in three dimensions, Comm. Pure Appl. Math. 57 (2004), 99-125, http://dx.doi.org/10.1002/cpa.10113. MR2007357 (2004i:35074)

[35] Tristan RiviÈre, Line vortices in the U(1)-Higgs model, ESAIM Contrôle Optim. Calc. Var. 1 (1995/96), 77-167 (electronic). MR1394302 (97g:58043)

[36] _ Dense subsets of $H^{1 / 2}\left(S^{2}, S^{1}\right)$, Ann. Global Anal. Geom. 18 (2000), 517-528, http://dx.doi.org/10.1023/A:1006655723537. MR1790711 (2002e:58030) 
[37] ETIENNE SANDIER, Lower bounds for the energy of unit vector fields and applications, J. Funct. Anal. 152 (1998), 379-403, http://dx.doi.org/10.1006/jfan.1997.3170. MR1607928 (99b:58056). Erratum: ibid. 171 (2000), 233.

[38] - Ginzburg-Landau minimizers from $\mathbf{R}^{n+1}$ to $\mathbf{R}^{n}$ and minimal connections, Indiana Univ. Math. J. 50 (2001), 1807-1844, http://dx.doi.org/10.1512/iumj.2001.50.1751. MR1889083 (2003f:58033)

[39] Leon Simon, Lectures on Geometric Measure Theory, Proceedings of the Centre for Mathematical Analysis, Australian National University, vol. 3, Australian National University Centre for Mathematical Analysis, Canberra, 1983, ISBN 0-86784-429-9. MR756417 (87a:49001)

[40] Michael STRUWE, On the asymptotic behavior of minimizers of the Ginzburg-Landau model in 2 dimensions, Differential Integral Equations 7 (1994), 1613-1624. MR1269674 (95g:35057a). Erratum: ibid. 8 (1995), 224.

Giovanni Alberti:

Dipartimento di Matematica

Università di Pisa

largo Pontecorvo 5

56127 Pisa, Italy .

E-MAIL: alberti@mail.dm.unipi.it

SISTO BALDO:

Dipartimento di Matematica

Università di Trento

via Sommarive 14

38050 Povo (Trento), Italy .

E-MAIL: baldo@science.unitn.it

GIANDOMENICO ORLANDI:

Dipartimento di Informatica

Università di Verona

Strada le Grazie 15

37134 Verona, Italy .

E-MAIL: orlandi@sci.univr.it

KEY WORDS AND PHRASES: Ginzburg-Landau functional, jacobians, integral currents, flat convergence and compactness, $\Gamma$-convergence, minimal surfaces 2000 Mathematics Subject Classification: 35B40 (35J65, 49J45, 49Q15, 49Q20)

Received: February 16th, 2004; revised: March 31st, 2004. 